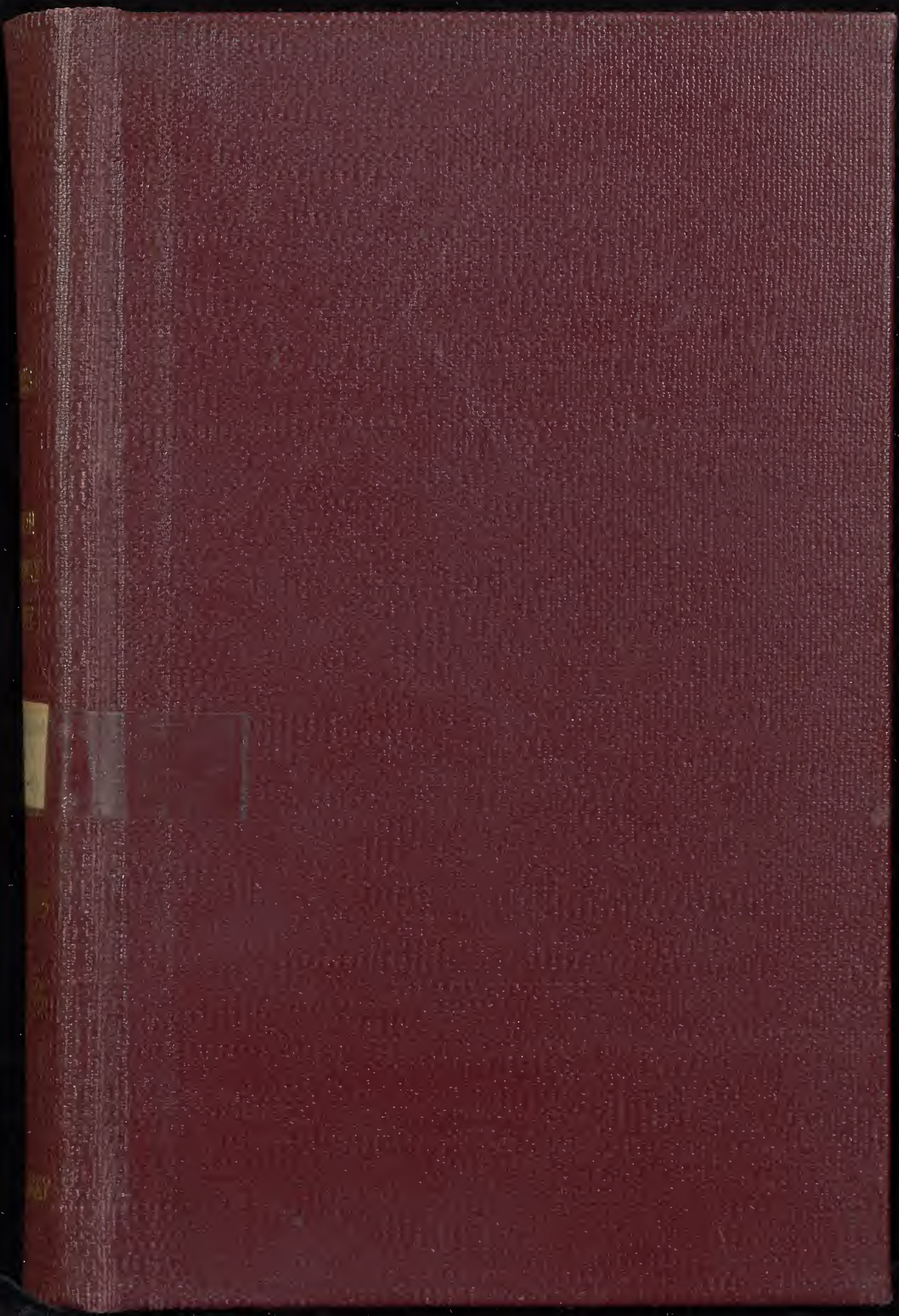




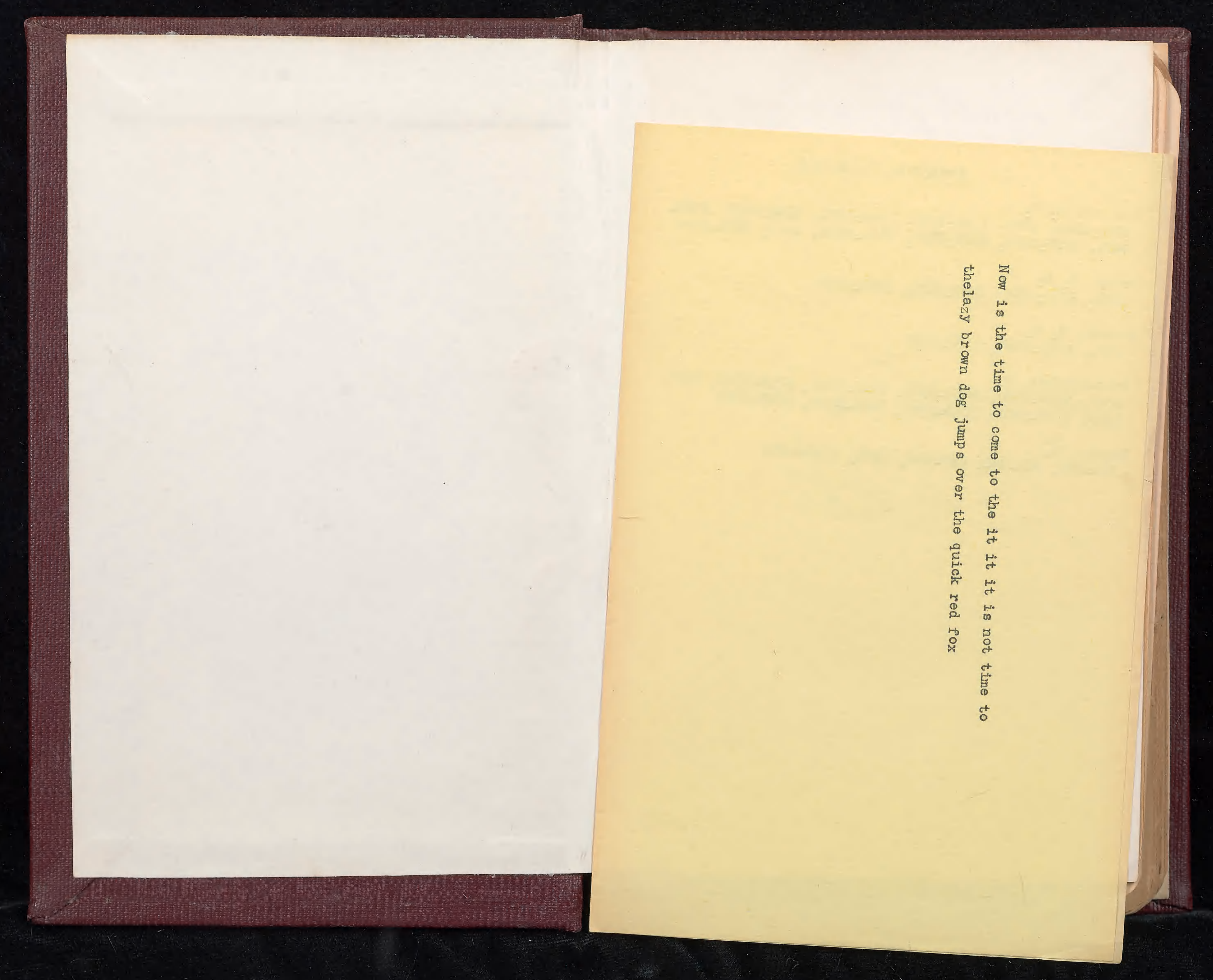


Holzner, F. X.

$1561-1580,1659,1712-1741,1949-1971,2008-2030,2058-$ $2661,2075-2079,2159-2181,2314-2318,2477,2645-2653$

McVay, H. E.

$1693,1711-1745,2032-2058,2062-2074$

Nerton, E. C.

$1151,1163-1165,1176-1187$

Schoenefeldt, L.

$2828,2872-2952,3044-3108,3157-3194,3216-3312,3344 m$ $3360,3400-3480,3538-3641,3664-3764,3803-3857$

Wagner, F.

$914-920,957-960,967-996,1061,1140-1146$

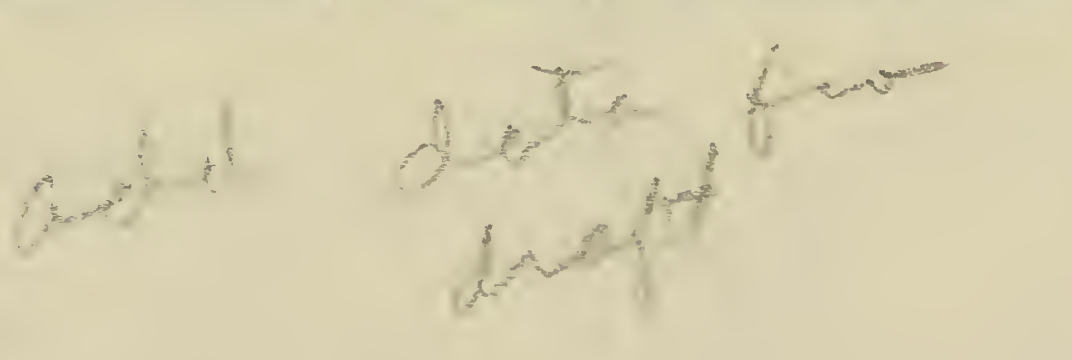




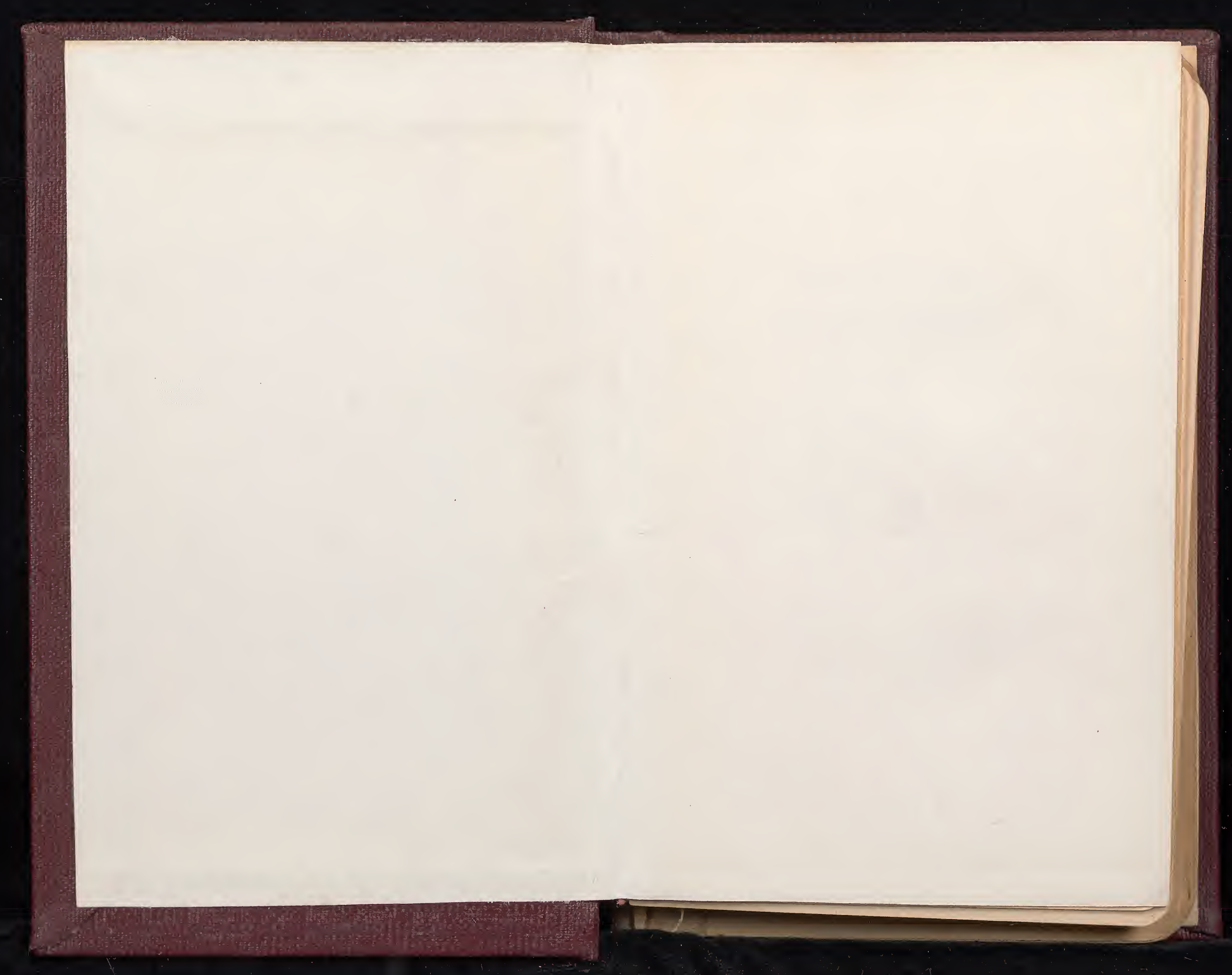




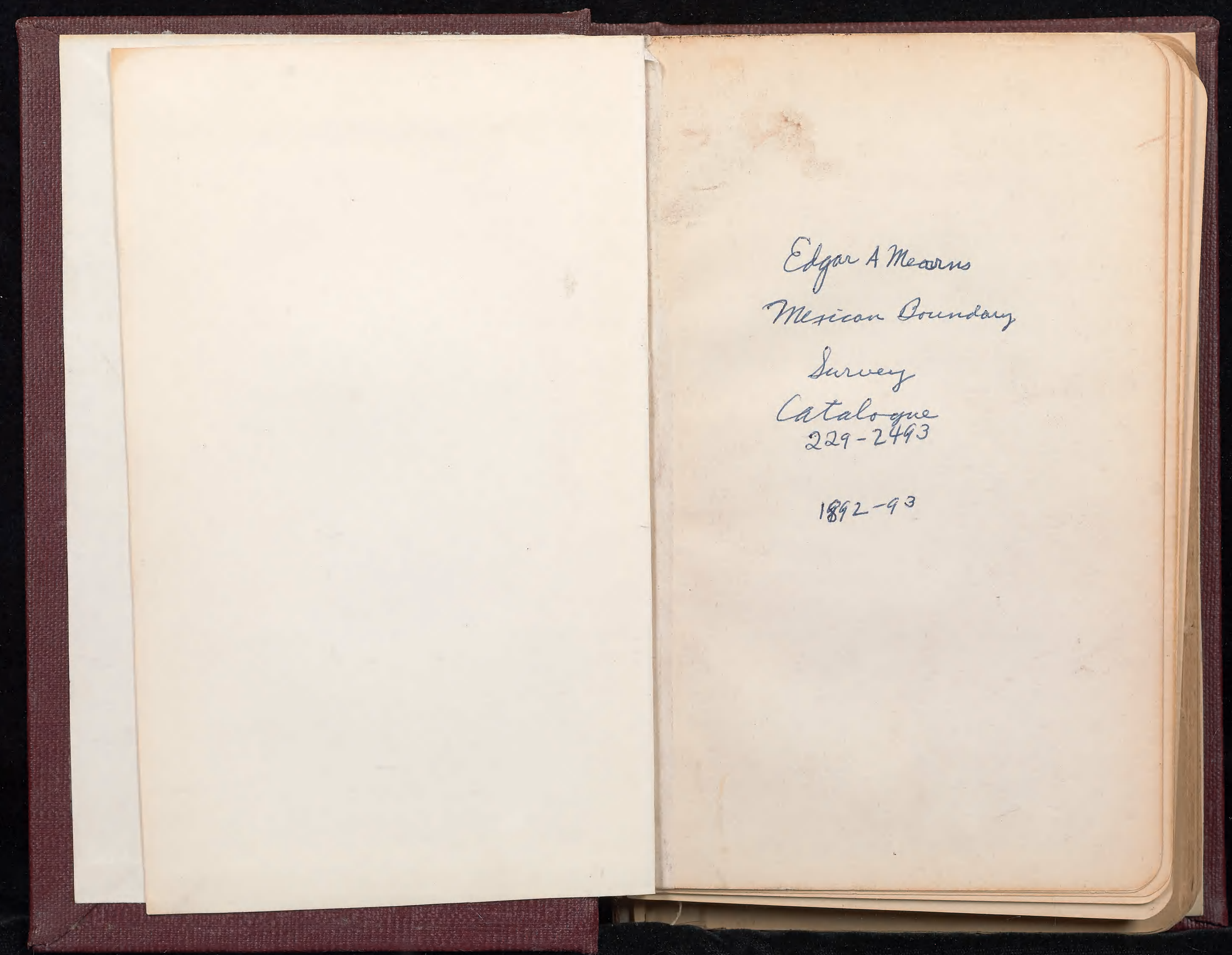




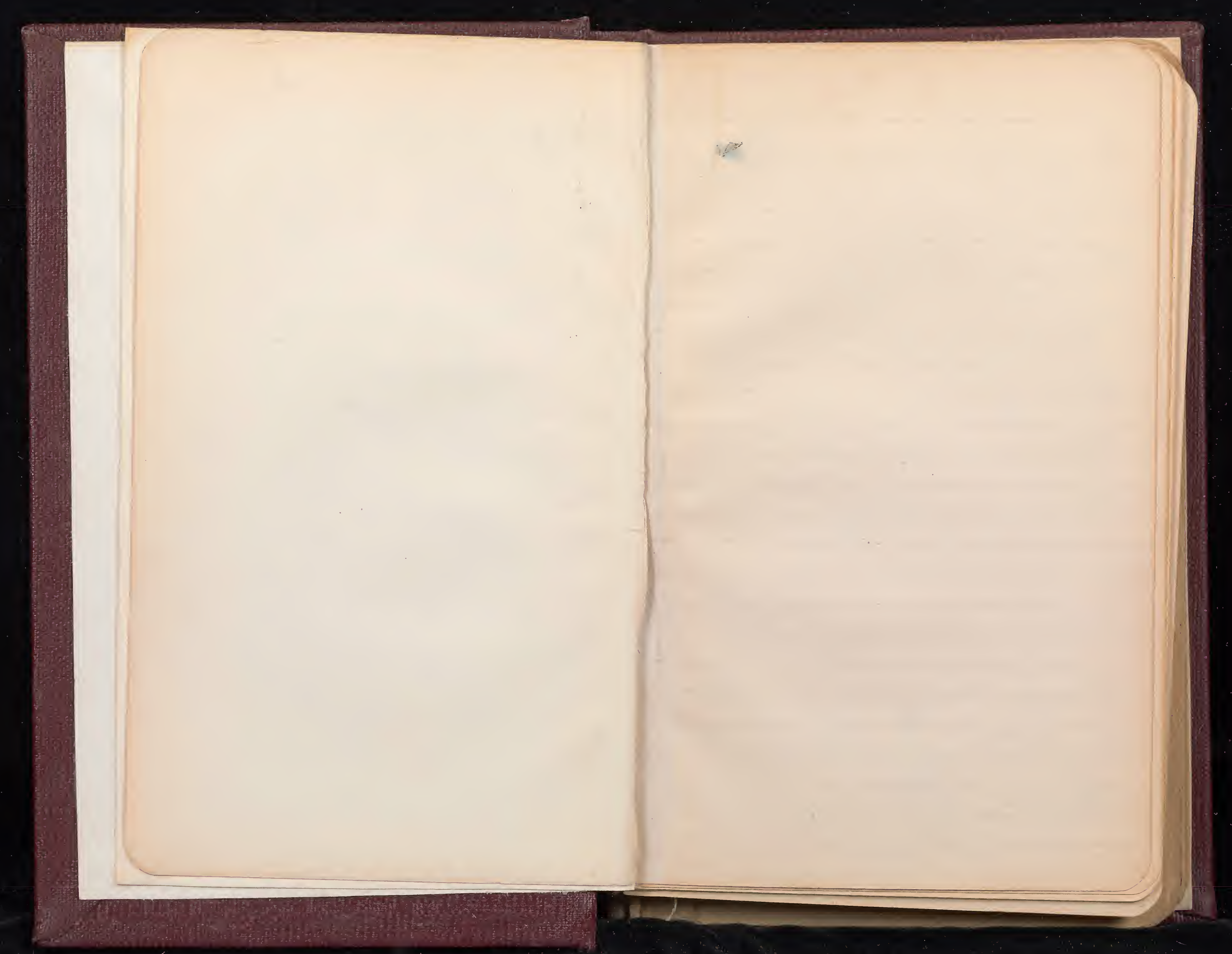




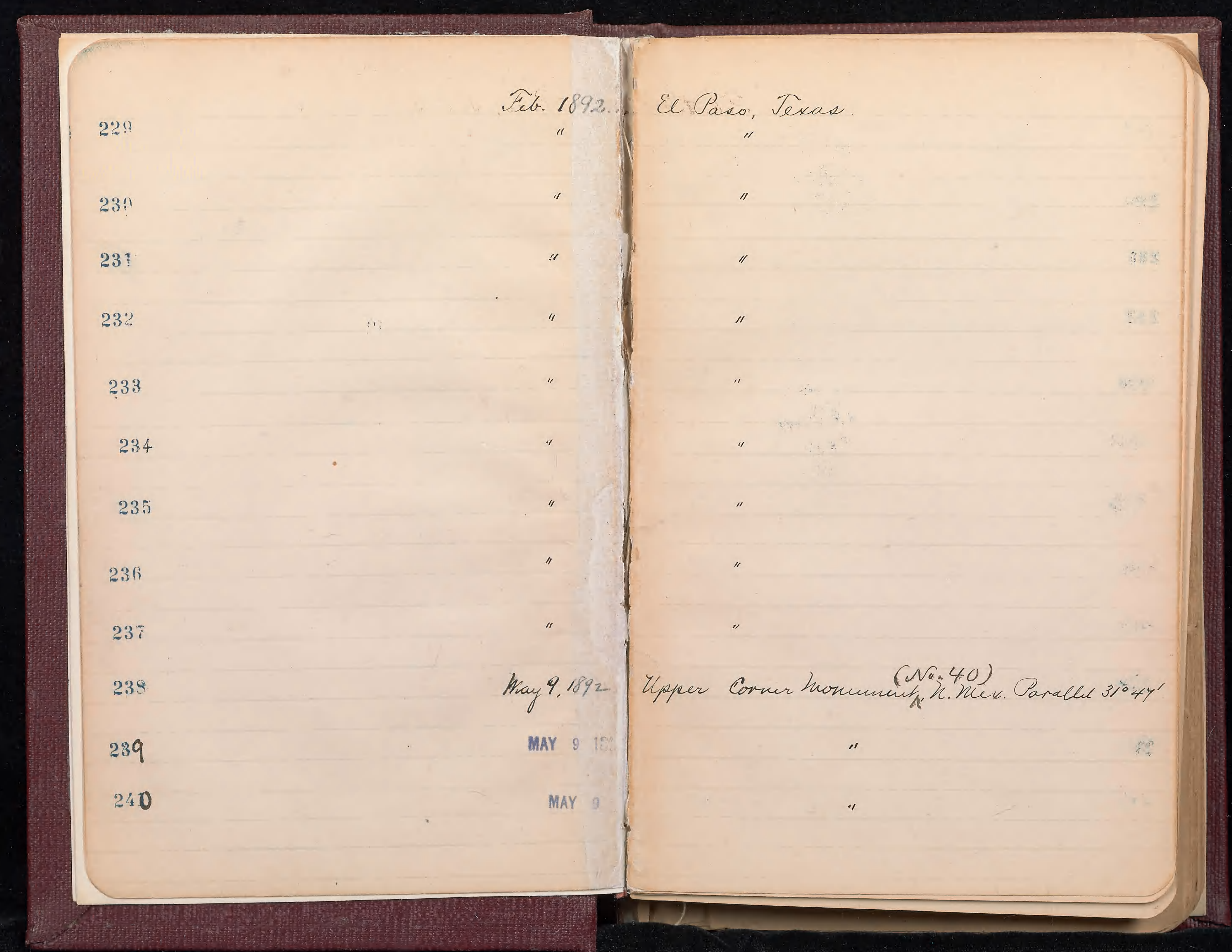




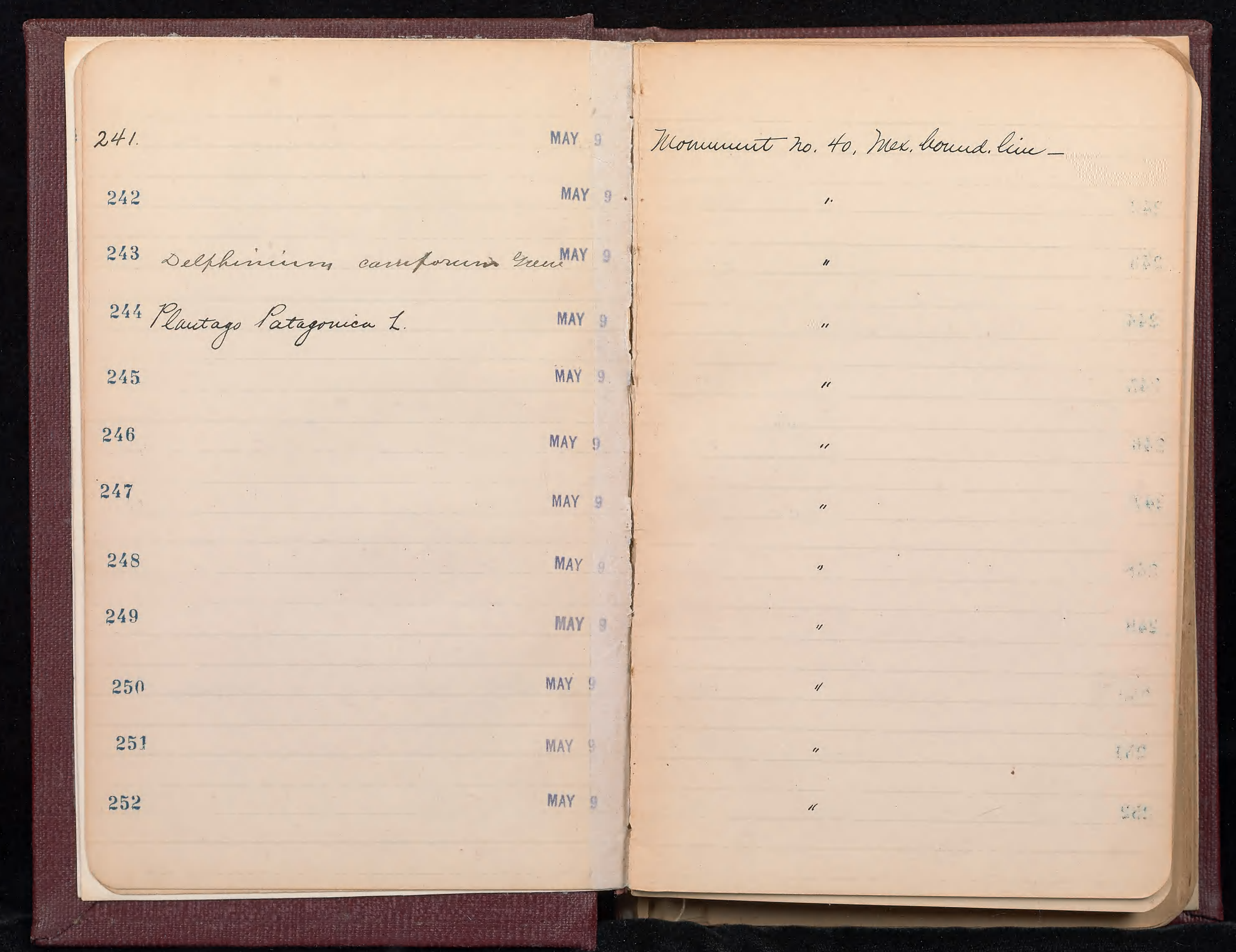




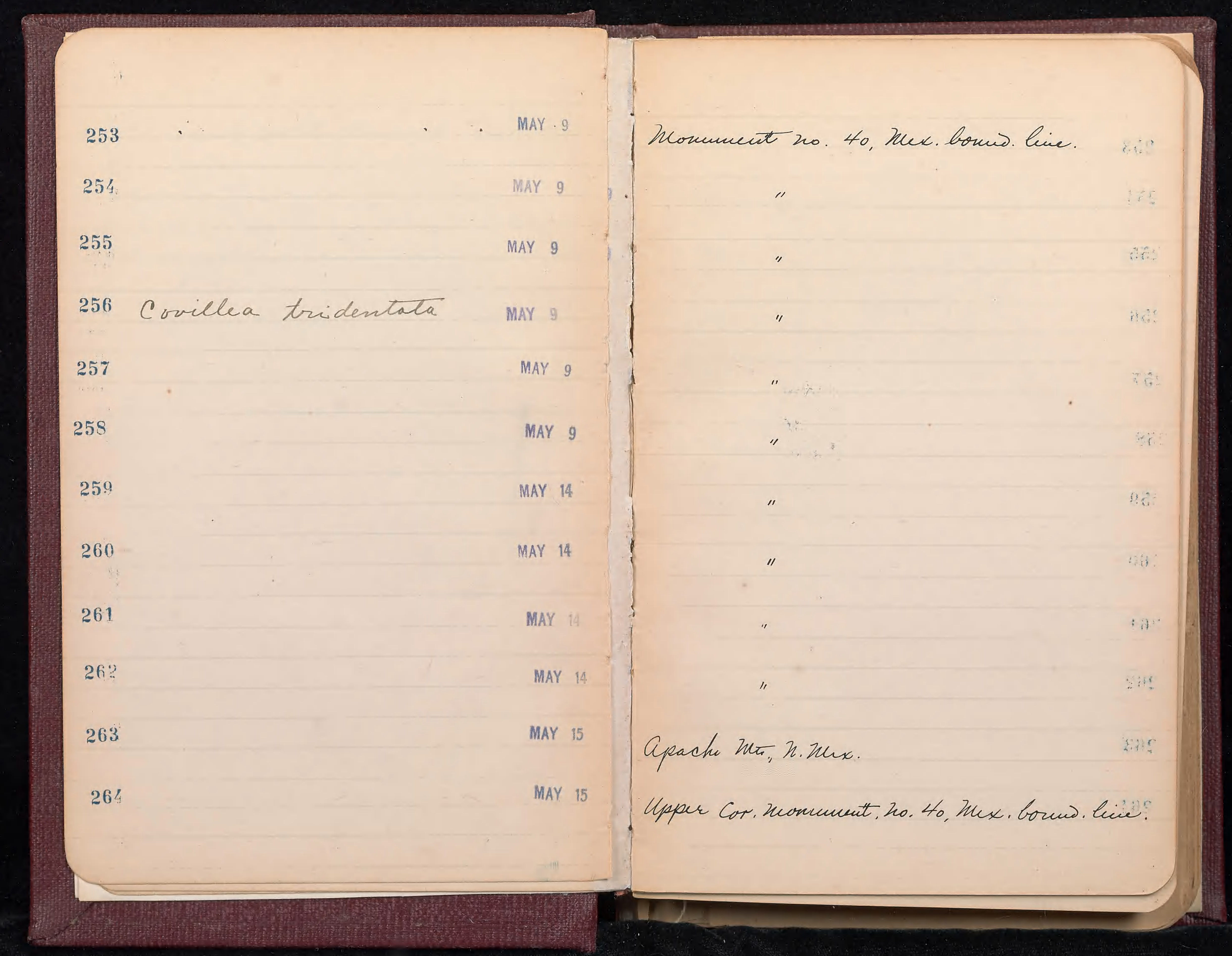




\section{MAY 31}

272 
278

278

279

280

281

287

283

284

285

28 ह

287

288
IUN 4 Emory Spring, foot of Euvry Peat, Graut leo, K. Wex.

IUN 4 Dog Spring, Lrant leo" Kew Mexico.

\section{JUN 4}

JUN 4

Doy hountains, Eract Co, Keer hexico.

IUN 4 Doy Apring, Erant leo, Hew Musico

JUN 4

JUN 4

JUN 4

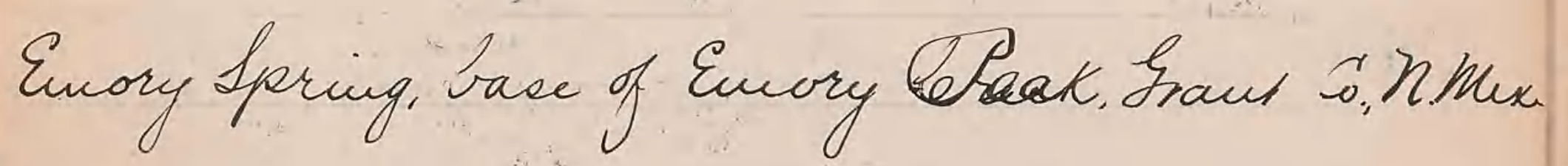
JUN 4 Doy hato, K. Hexico, niar honument to. JUN 4 Emory Peak, Irant Co, Kew Hucico.

JUN 5 Dog Spring, Yraut Co, Hew Hexico. JUN 5 
289

290

291

292

293

294

295

296 Umiperus pachyphtoea Zorr.

297 Minulus

298 Querens

299

300
Juiv id

JUN 10

un 10

JUN 10

JUN 10

IUN 10

JUN 10

JUN 10

JUN 10

JUN 11

JUN $\cdot 12$

JUN 12

White Water, Ghifunahua, har U. S. Gound. hive. White Water, bhilunatura, man U.S. bound. Gin. Mnxicue boundary Gie, mar White Water, Chihuahiua.

Dog hountains, Kew hesico.

"

Doy hountains hear sog Lpring, Kew husico. Dog Apring, Hew Hesico

San Luis homitains, hear hexicqu bound. Cinien.

Dog Monutains, mar Dog Spring, Hur Nexico. 
301

302

303

304

305

306

307

308

30 ?

310

311

312
JUN 12 Doy Konutains, Kear Dog Lpring, Hew Kuxico.

JUN 12

IUN 12

JUN 12

Dog Spring, New mexico.

IIII 12 Zrant Co, Kew hesico, north of Euvory Peakt.

IUN 12 Sumnit of Dog homitains, Heer huxico.

IUN 12 Lrant leo, New Nexico, north of Enory Peak.

JUN 13 Doy Spering, Kraut Co, New huxico.

IUN 13

JUN 18

JUN 13

JUN 13 
313

314

315 Cisclepias

316 Eschscholtzia

$31 \%$

$-318$

310

320

321

$3 \% 2$

323

324
JuN 13

JUN 13

JUN 17

JUN 17

JUN 17

JUN 17

IUN 17

JUN 17

JUN 17

JUN 17

JUN 17

JUN $\$ 8$
Dog Apring, Kew kesico.

ats

Wh

White Water, Chihuahura, Hex., Hear U.S. bound lim

" on boundary liue.

918

तो

Bonudary liue, near White Water, lehihuativa.

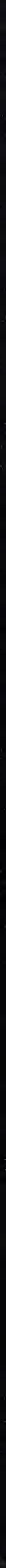

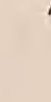

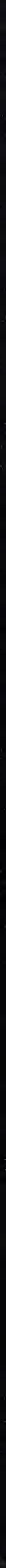

" 


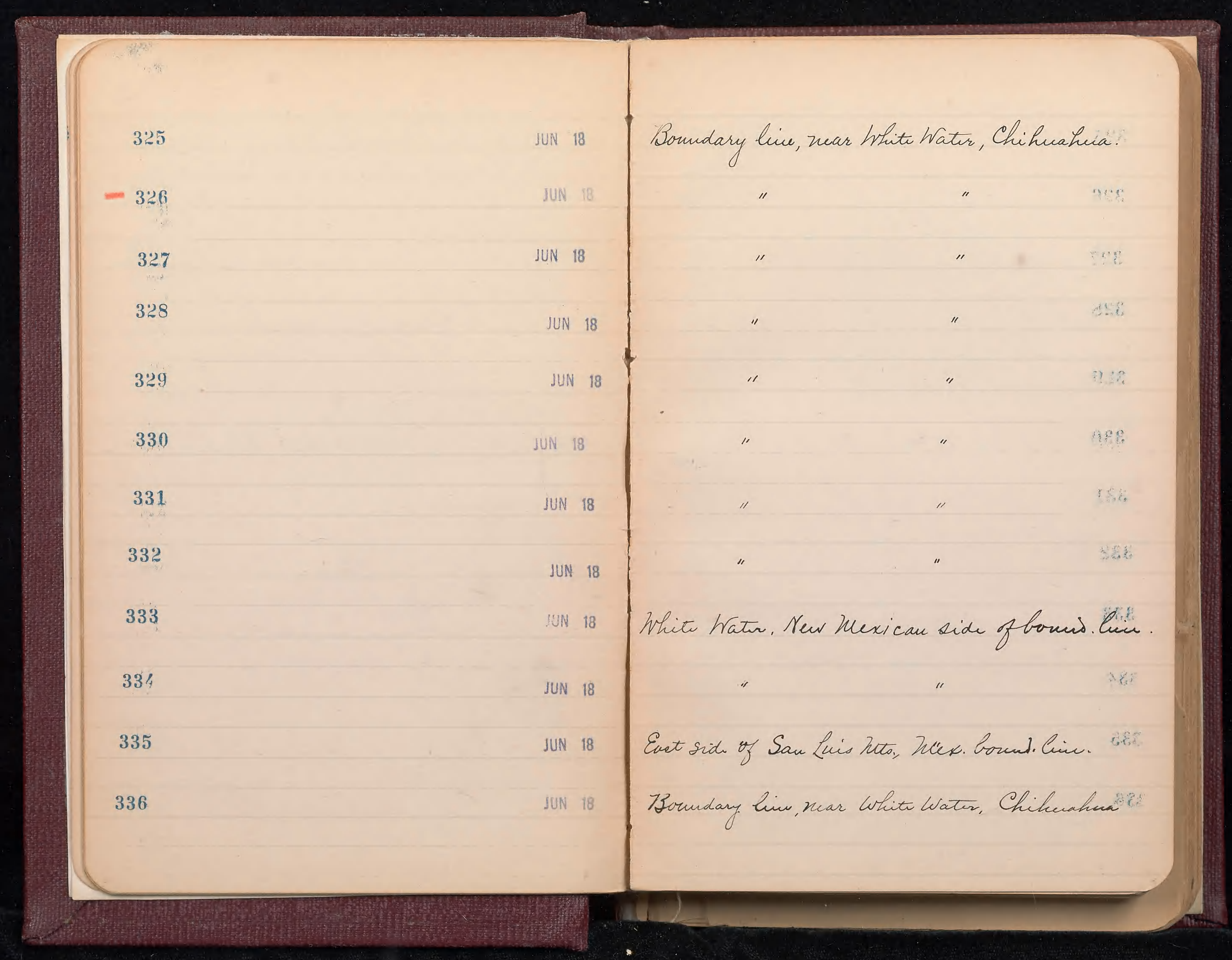


$33 \%$

338

$-339$

340

341

$-342$

$-343$

344

345

$-346$

$-347$

- 348
JUN 18

JUN 18

JUN 18

JUN 18

JUN

JUN 18

WN 18

JUN 18

JUN I8

JUN 18

JUN 18

$4 N \quad 18$
White Wator, Chihuahua, on bonudary hive

$$
\text { "Nur Mexican side of boundary him. }
$$

$$
\text { I }
$$

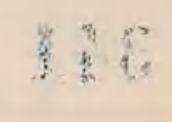

" hear the Muxican side of bonid. live. " $" 1$

11

II

21

" Hew Herrican side

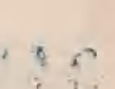

"

(1) 


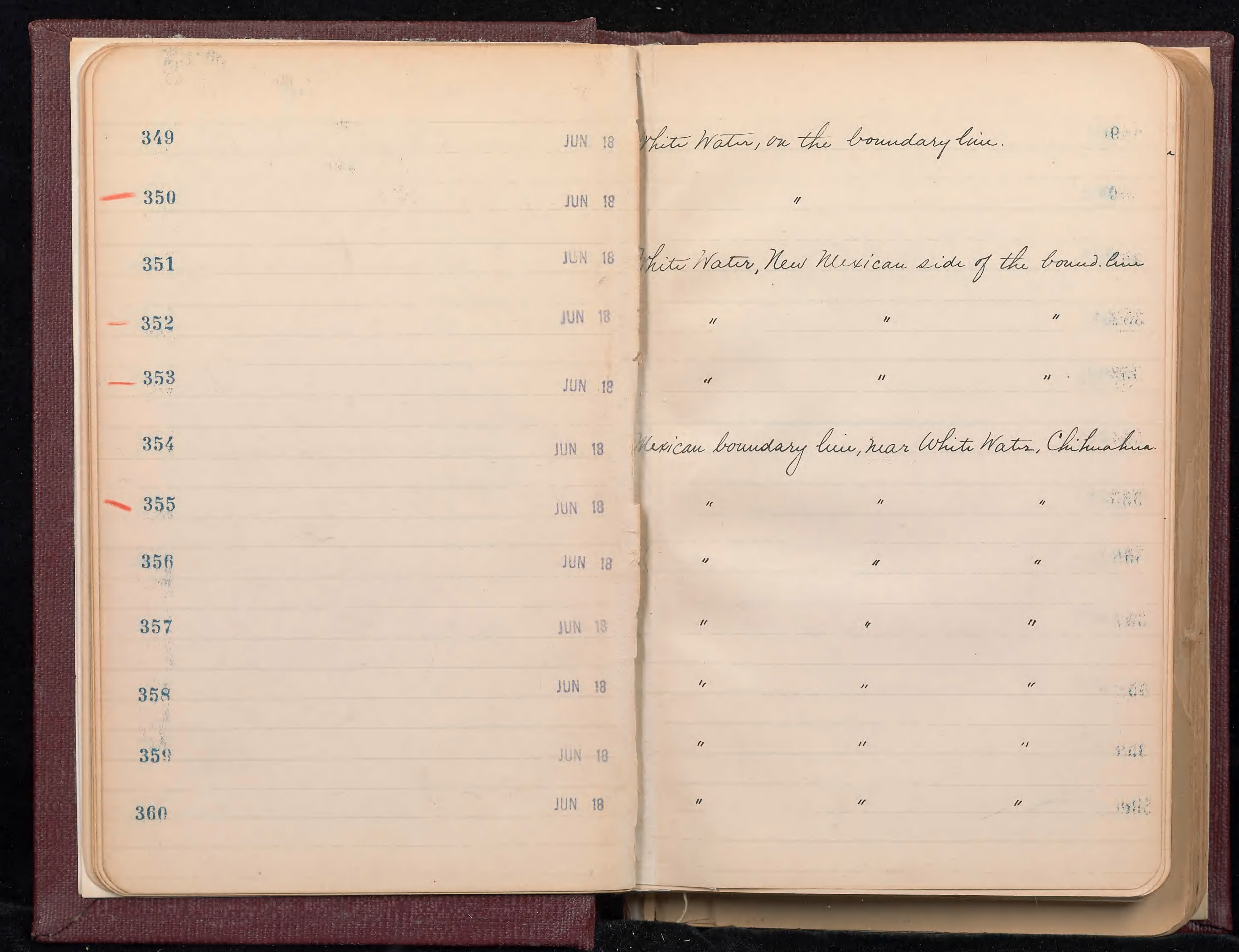




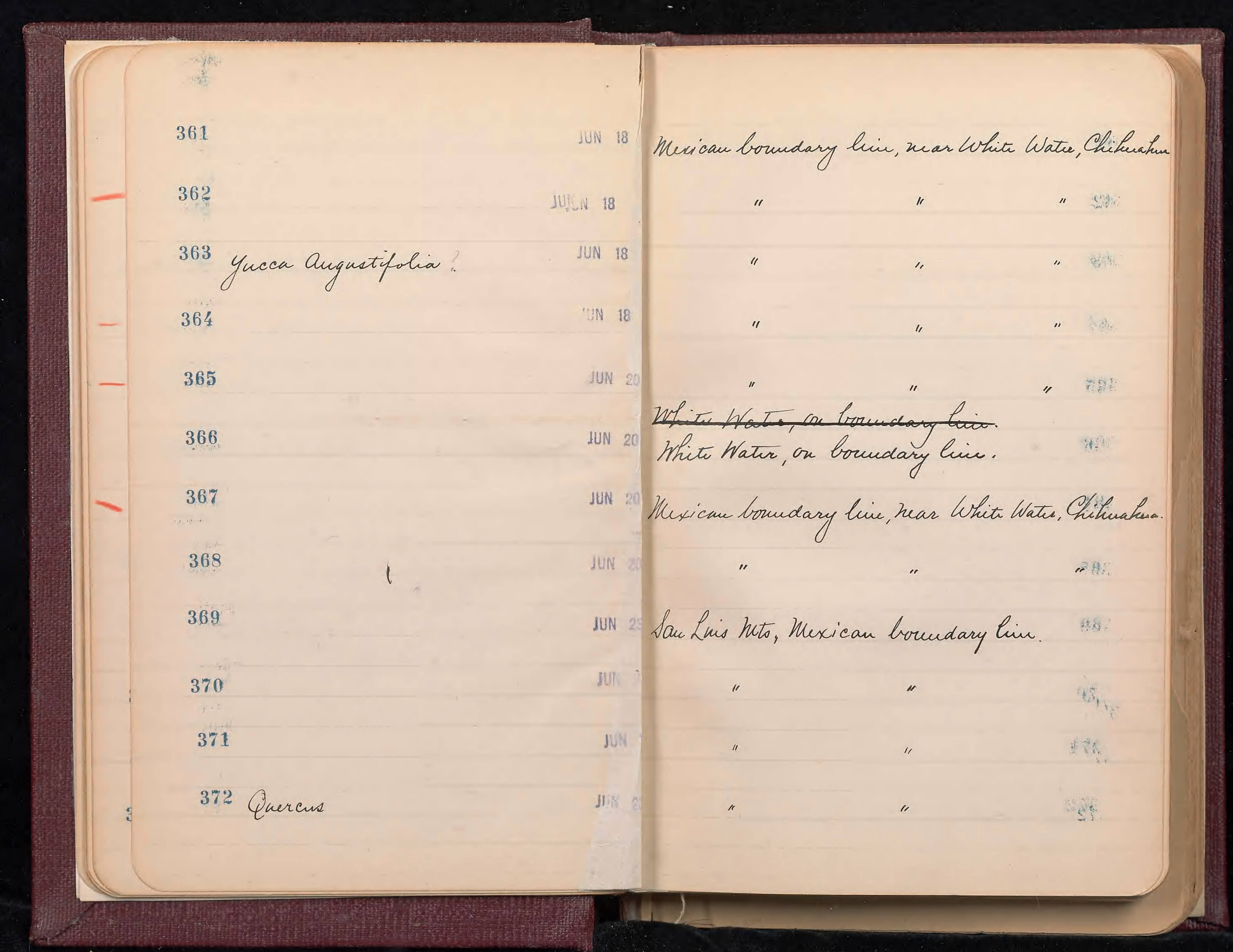




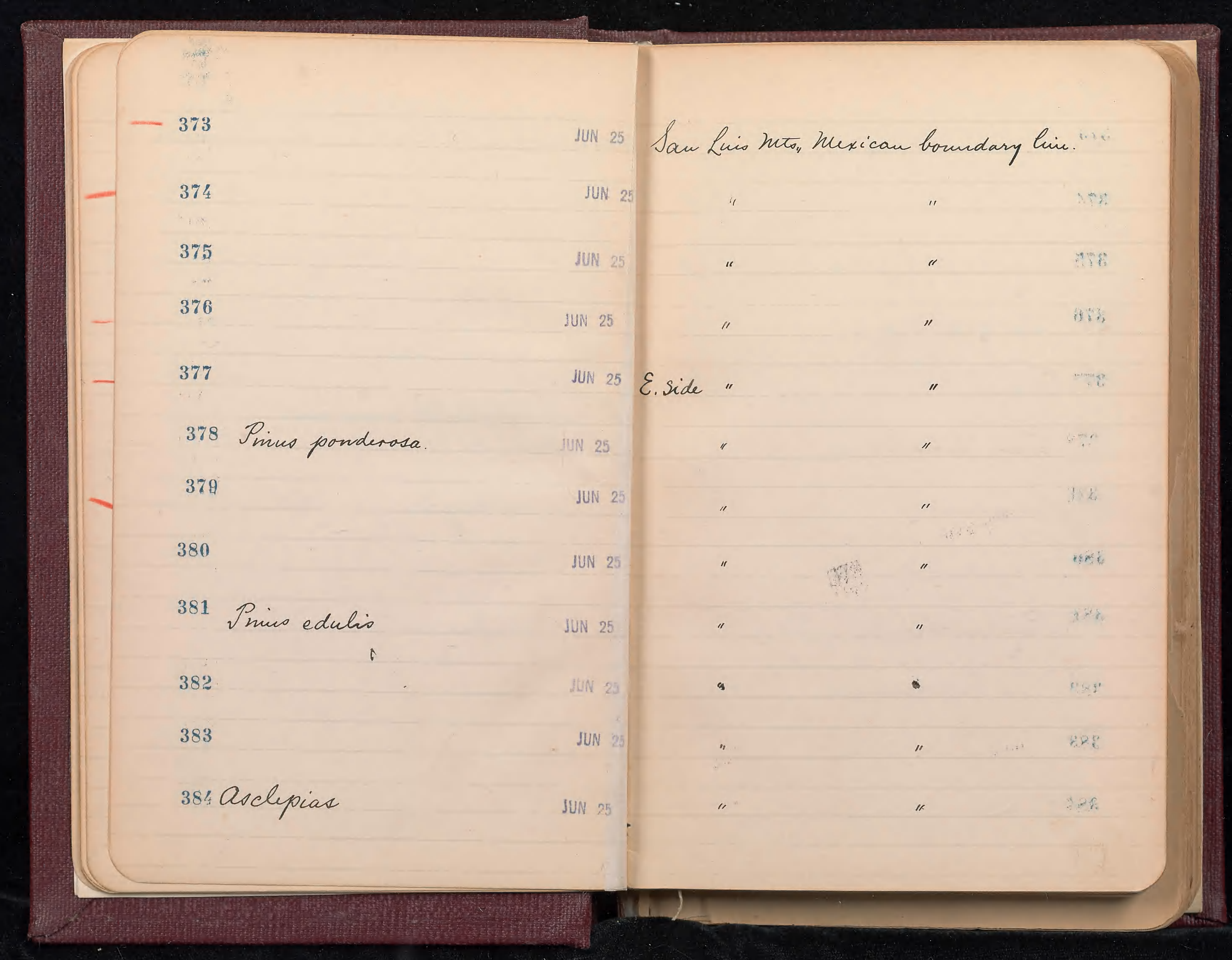


385

386

387 funiperus pachyphlvea. Lorr. 388 Pint honilifura

389 Cnicus

390 Jinus Chihuahuawa

391 Phoradudrow

392 Asclepias

393 Platanus Wrightii.

394 Yhus toxicodeudron. 2

395 Zuglans rupestris

$$
396
$$

JUN 25 SauLuis Ht, Nexican boundary lin.

Juiv 25

JUN 25

JUN 25

JUN 25

JUN 29

$J U_{4}^{2} \quad 1$

JUL 1
JUL 2

JUL 2

Cajou Creete, Chihuahua, hear bouns. Cuin.

(E.sich)

"

2

" Chicuahua.

JUL 3

JUL 2 
397

398

399 Populus Frewonti

400

401 Prumes

Cones. 402 Psendotsuga taxifolia.

403

404

$-405$

406 Quercus

407. Quercus

408 Qurcus.

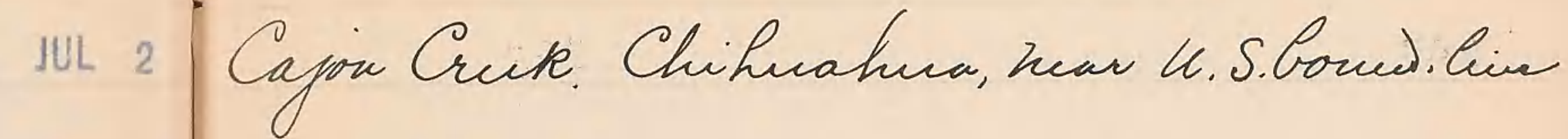
UL 2

JUL 2

JUL 2

JUL 2

IUL 4 SanLis nontanis, Chihuahua.

JUL 5 Sumint of midde peak of Cuimas Hets, Hew Mux.

JUL 5 - Lan Lis hoto, Kiexican bound. Lic.

If

HUL 5

II

JUL 5

"

"

$T^{2}$ 
409 Quercus

410 Peudotenga.

$a_{\text {ovms. }_{\text {s. }}} 411$ Quercus.

cones 412 Prims pouderosa Dougl.

cones 413

4ls Peudotsuga.

415 Prius

416

417

418 Pnim ponderasa Doryl.

419

420
JUL 5

Lan Lis Hto. Hexicau boundary liec

JUL 10

IUL II Smmuit of San Luis hts, Chihuahwa.

JUL.

JUL II

JUL II

JUL. II

JUL 11

IUL II

San Luis Het, near Saw Lnis Spreng.

JUL II Sumint of San Luis hets, hear hex. bound hive.

ll 
421

$42 \%$

423 Pmix nonophylea Torr. Prew.

424 Inius ponderosa Dongl.

425 Pendotonga

426

427

428

429

430 Pries

431 Quercus

432 Qurcus IUL 11 In a cauvn half way up the San fuis hits. Close to

JUL II Sumuit of Law Lis Mts, Mex. bound. Eur.

JUL 11

JUL. 11

JUL. II Sau Luir Juts. Chicherahua, Kuxico

JUL 11

III II San Lis Mto, Mexican bonud. Pin.

JUL $\|$

JUL. II

Sumnit of Saw Luis hto, Mexican bound. hive.

I'L 11 Sumuit of highest peeate of Sau Luis Ints.

JUL 11 Sau Lub Nto on Nexican bomos. hir 
433 Quercus

434 Quercus

435 Ruercus

436 furcus

437

438 Prins pouderosa Dragl.

439 Oxytropir.

440

441 Pecudotinga

4.22 Populus tremuloides.

443

444 Querene
JUL 11 Sumnit of San Luis hts., 2 n. S. of hex, bound. hine.

JUL III

Hux. bonnd. hio

JUL 11. San Lies Monutans

JUL. 11 Sumnit of Las Luis hits.

vil 11 Sighest sumuit of the San Luis hito.

sul Sammit of Anviuas Hits, Herr Nlexico.

III. 12

JUL 12 Anmias Peak, S. W. K. mexico.

JUL 12 Sumint of Aurmas Hts, S. W. Kew Mexics. "I

JUL 12

II

JUL 12 


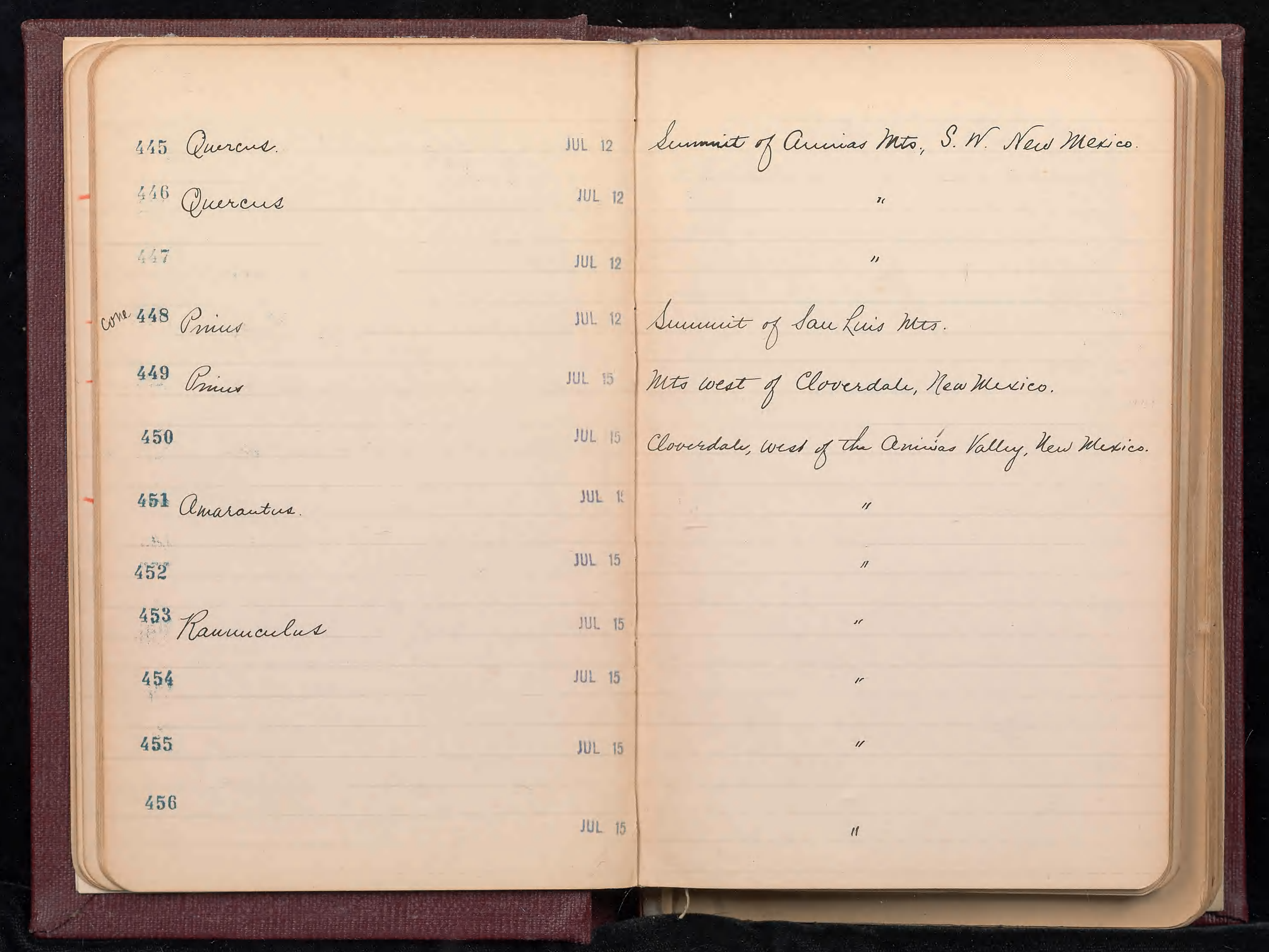


Acacia

458 Quercus

450 Pannuculue

460 Himulus

461

-403
463

464

46.5

406 Solidago

$46:$

478
JUL 15

JUL I5

JUL. 15

JUL 15

JUL 15

JUL 15

JUL. 15

JUL 15

JUL 15

JUL 15

JUL. 15

JUL 15

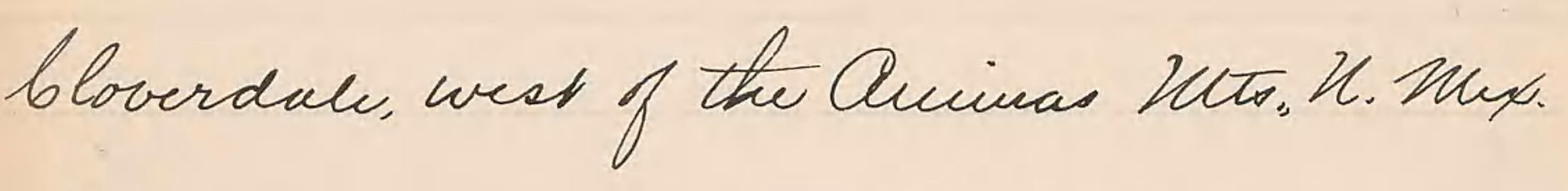

bloverdale, heer Hesico

"

"

Cloverdah, Aminas Valley, Keer Hexico.

Gnadelupe Canon, hear Coverdale, h, Hex.

Head of Swadelupe Canon, har Clorvedah, K. Her bloverdal, M. Huxico. 


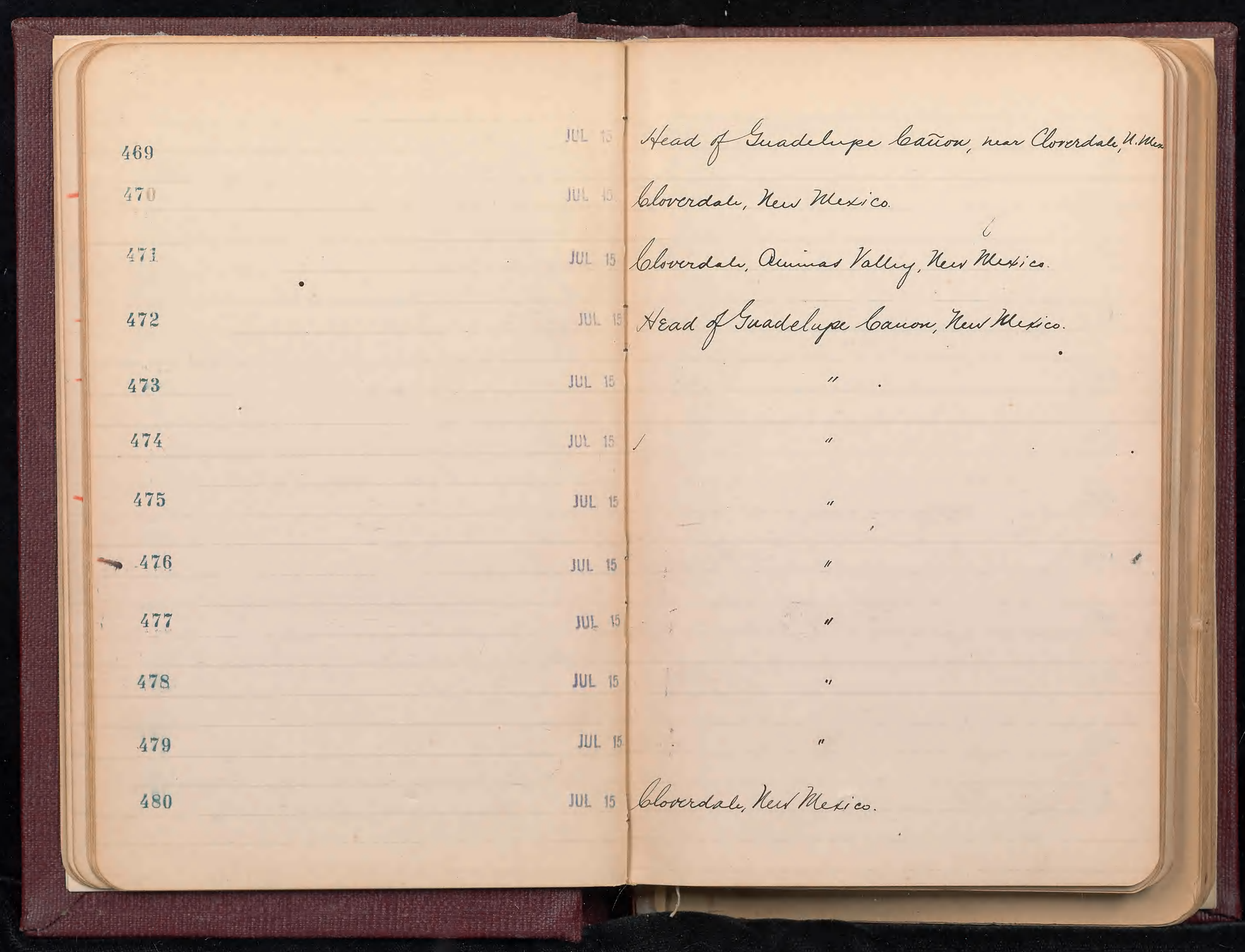




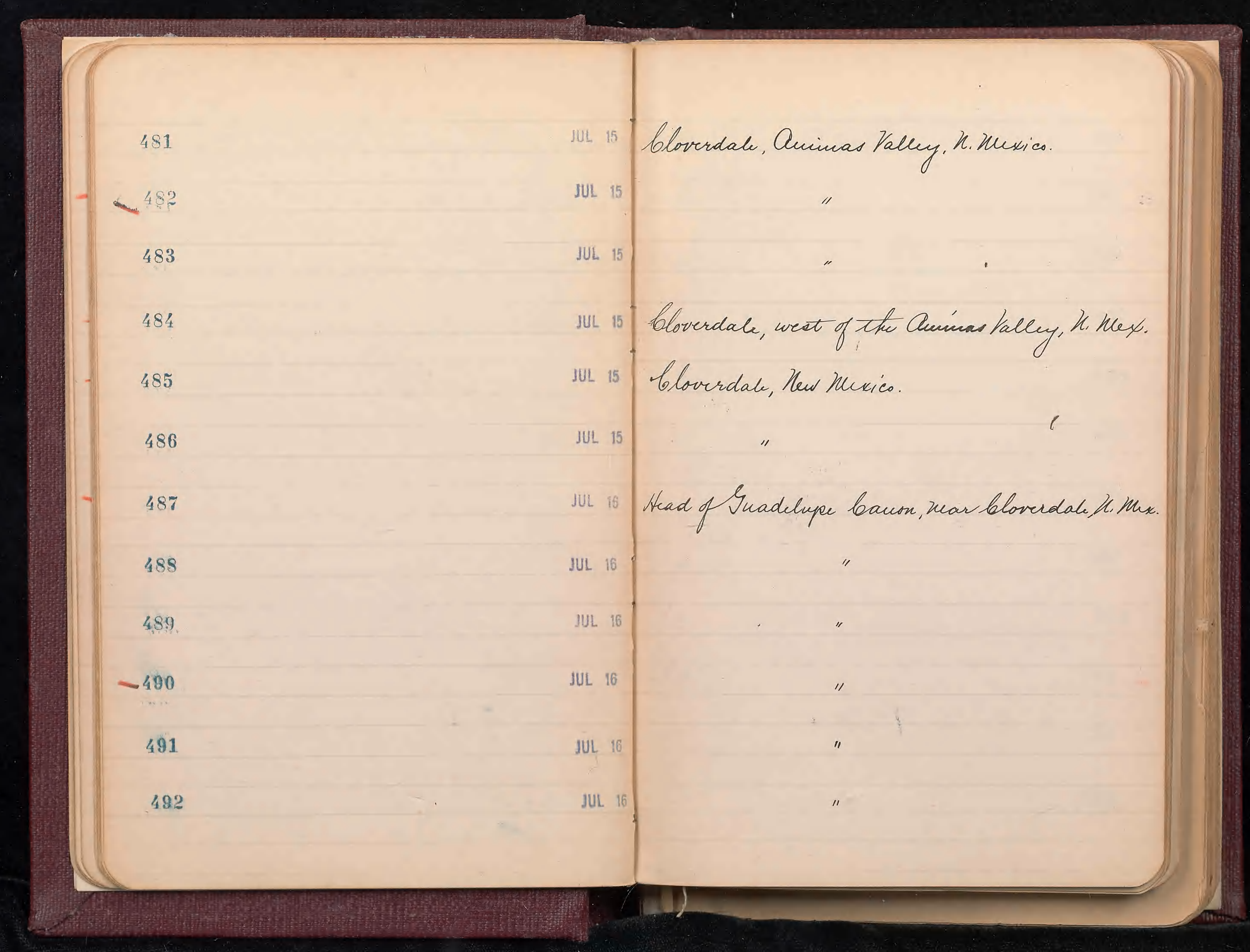




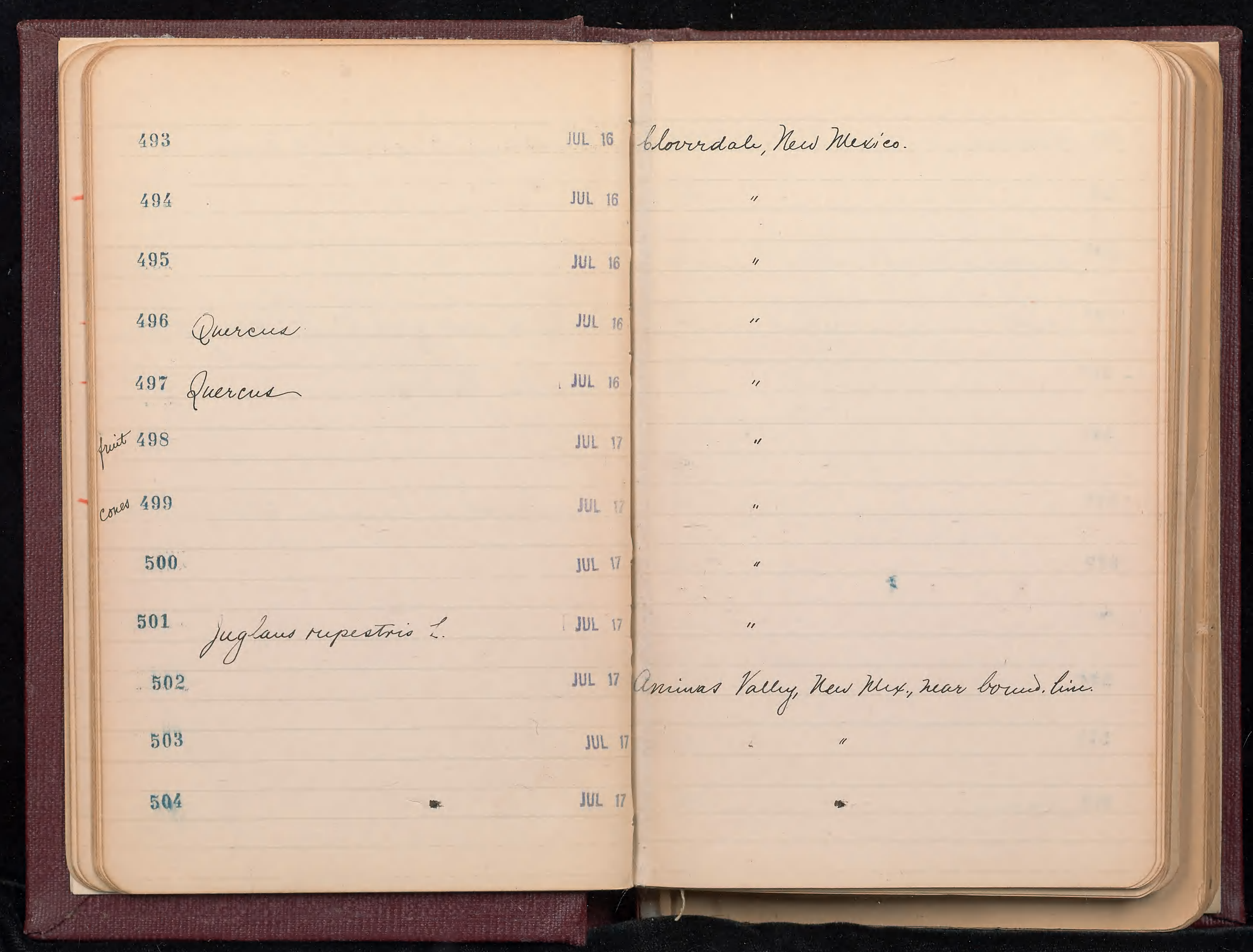




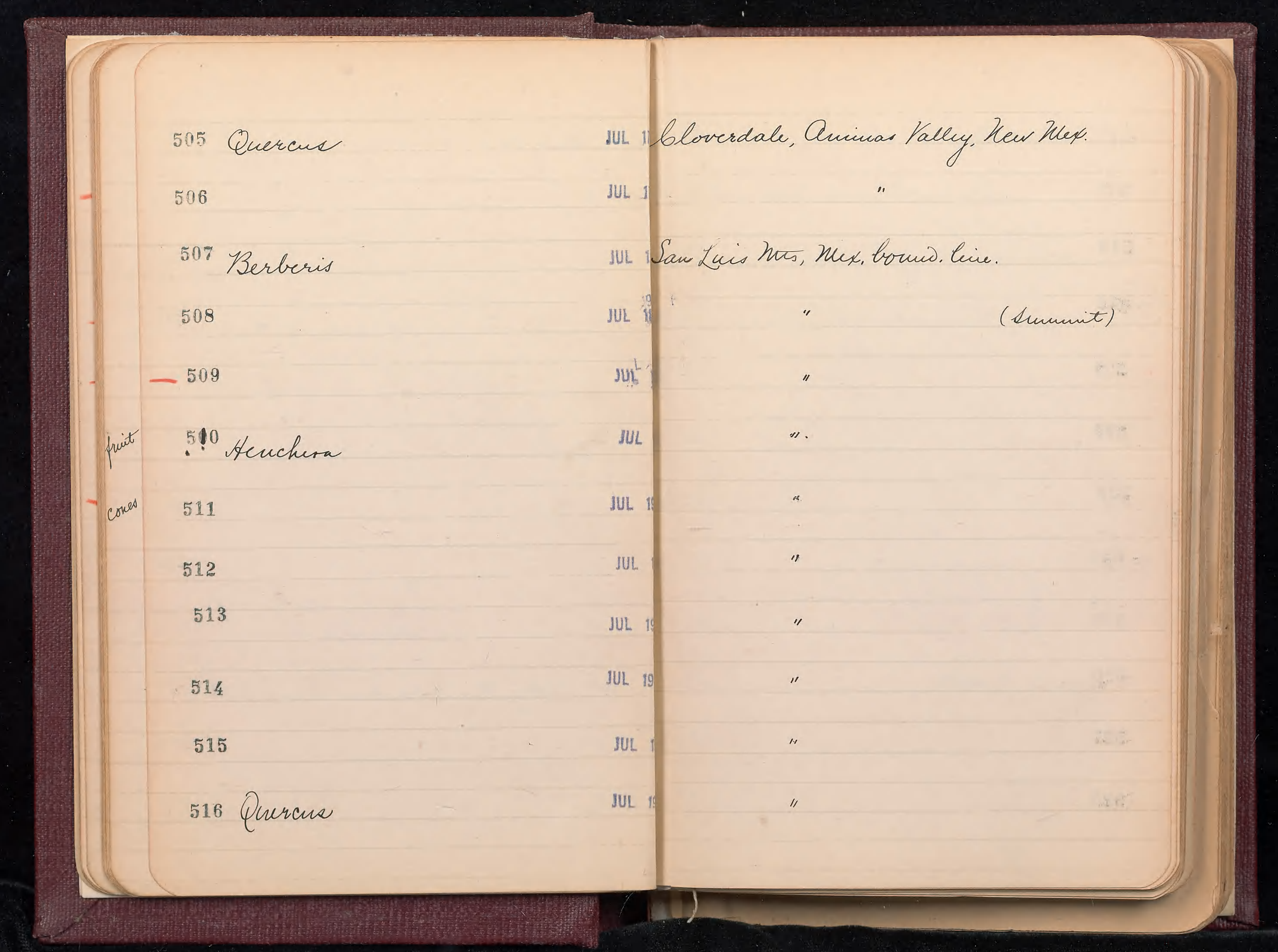




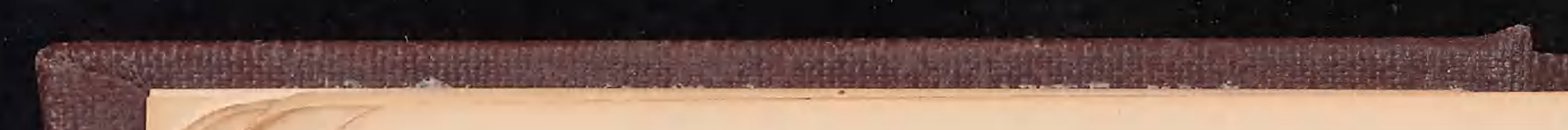

517 Quercus

518

519

$-520$

521

522

frit

cores -523

$-524$

525

526 Yuece baceata.

527

528
IUL is Summit of Saw Luis heto., hux. bond, liver. JUL is Lan Lus huss, Hux bonnd. Cinc.

JUL is Scloverdace, West of the Aminas Vally, Liner. JUL "San Luis Znts, Jux. bonud. line.

JUL 4

JUL

ปปL.

JUL

JUL 19

JUL

JUL

JUL
"

"

"I

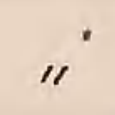

,

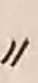

" 
529 Purcue

$$
530
$$

531

532

533

534 Quercus

535 Acer

536 Berberis

537 Zunóperus

538

539

540
JUL 19

Saw Lnis hos, on hux. boned. Cier.

JUL IS

JUL 18

JUL IO

JUL I

JUL

JU!

JUL

IUL

JUL.

JUL

JUL
"

"

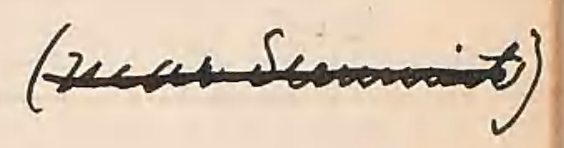

(near Summit)

"

4

(Souchern bour)

(neas summit) 


$$
\begin{aligned}
& 511 \\
& 542 \\
& 543 \text { Quercus }
\end{aligned}
$$

544

545

546

548

$5 / 8$

cone. 549

acorts.550 Quercus

worrs. 551 Quereus hypolecuea

coru. 55\% Psendotonga
IUL "San Lies nets. Mnican boundary Giv.
JUL

JUL

JUL

IUL

JUL

JUL

JUL

luk

IUL

JUL

JUL (huar Summint)

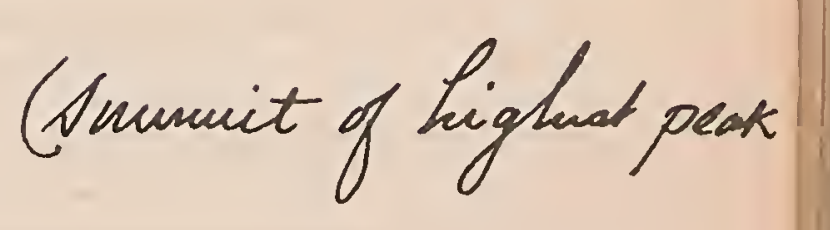




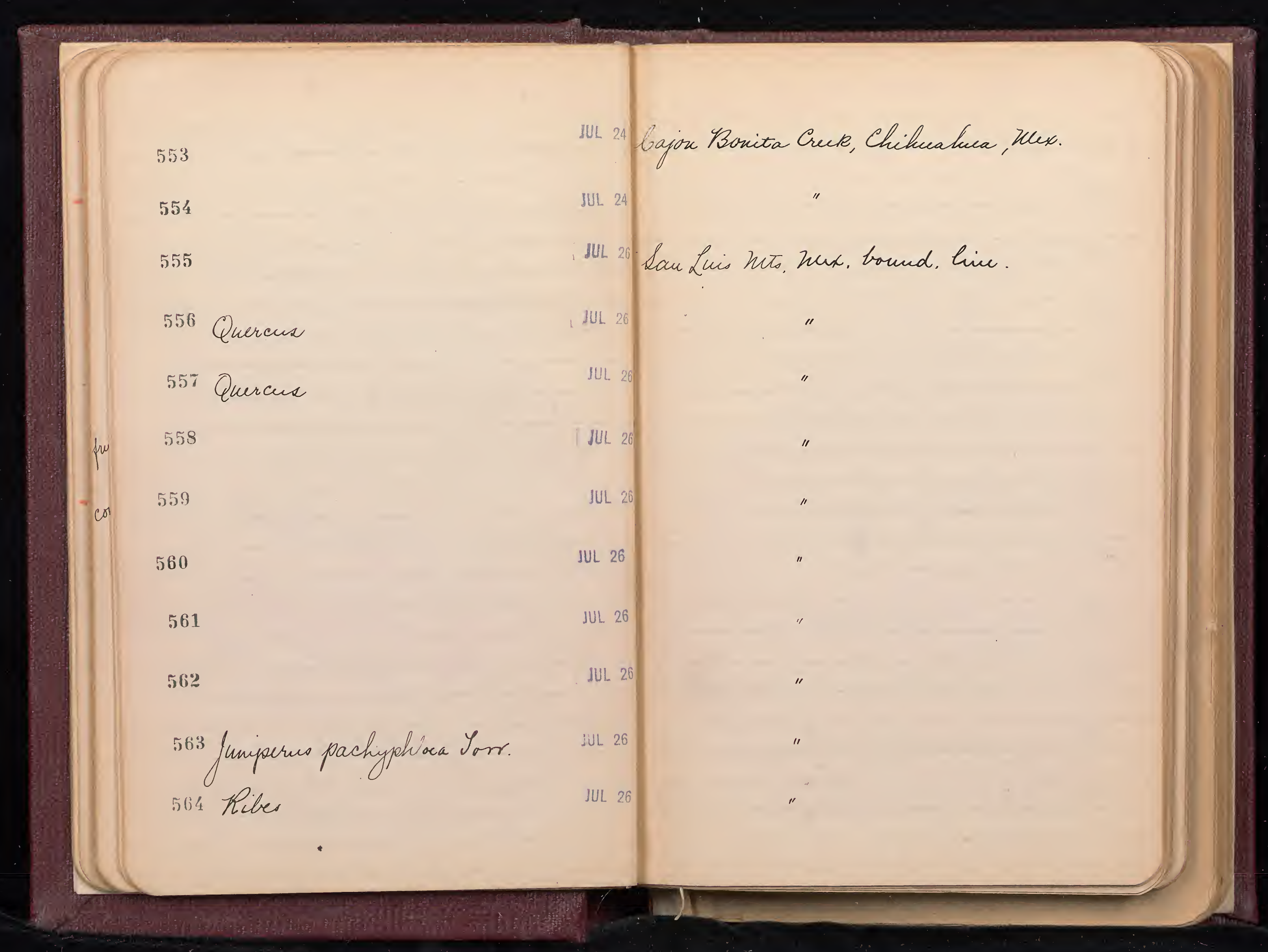


565

566

567 Thalictron Fendhri

568

569

570

571 Pinus Chihuahuaua.

572

573

574

575

576
JUL 26

JUL 26

JUL 20

JUL 26

JUL 26

JUL 26

JUL. 26

JUL 26

JUL 26

JUL. 27

JUL 27

JUL 27

JUL 27

San Luis Mrto, msicau bondary him.

II

"

II

"

"

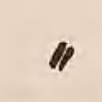

Animas Vally, Krw huxico.

Inadeloup banon. Kew Hexico.

Animas Vally, Hero Kuxic 
577

578 Salina

579

580

581

582

583

584

585 Tetanus Wightii.

586

587

588
JUL 27 Animas Rally, Kew Mexico.

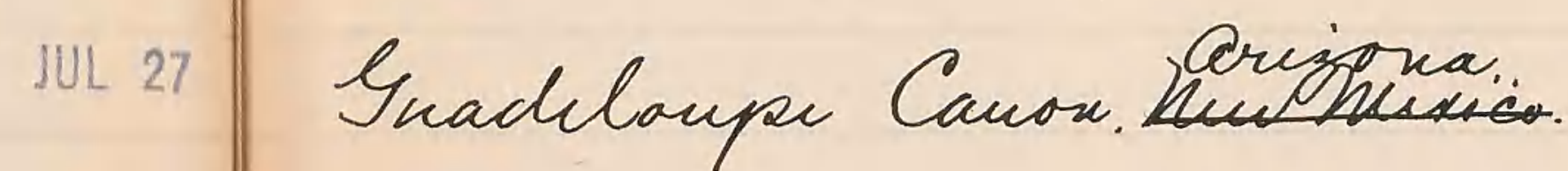

JUL 27

"Chilucuatiua

JUL 28

"New Mexico.

"Arizona

Kew Mexico.

Arizona.

JUL. 28

JUL 28

Hew Lucio.

JUL 28

"I

/l

JUL 30 San Beruardino Ranch, Arizona.

JUL 30 
589

590

591

592

593 Gassia Wislizeni A. Yray.

594

595 blematis drumenondir 7.PY, AUG 7

596

597

598

599

600

AUG 5

AUG 5

AUG 7

AUG 7

AUG

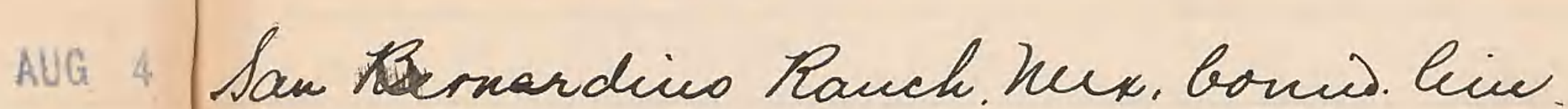

Hear Saw Keruardiess Rauch. Kux. bornd. Him AUG ILan Beruardius Rauch, Hexican bound. Lier.

AUG 7

AUG 9

AUG 12

AUG 12
11

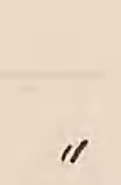

"

"

"

"I

(I)

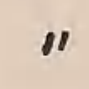




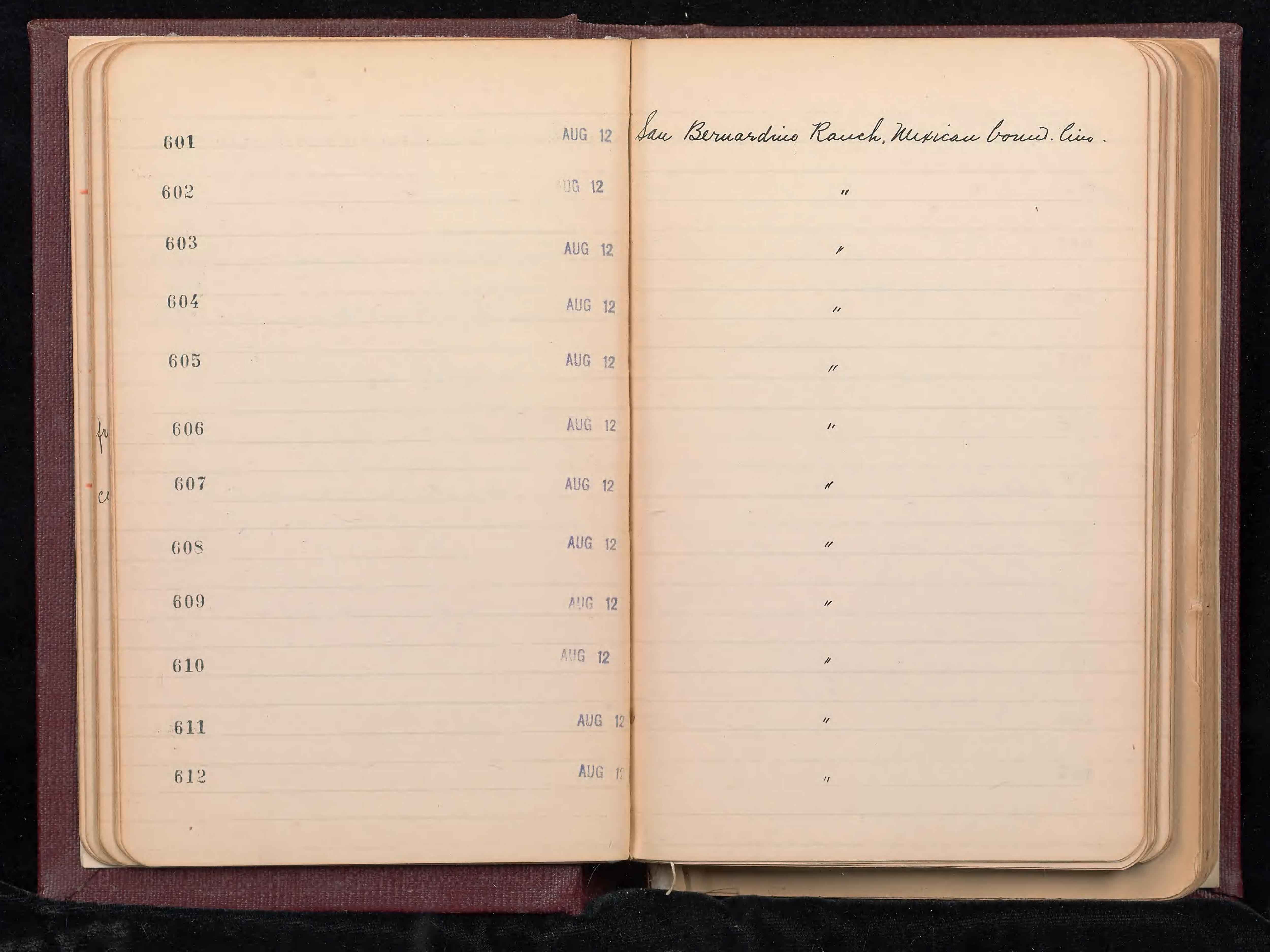


613

61.4

615

616

617

618

619 Solannue

620

621

622

623

624
Law Berwardiuo Rauch, Hex. bound. Bin.

\section{AllG 12}

AUG 12

AuG 12

AUG 12

A: 19

AllG 12

AUG 12

AUG 12

AUG 12

AUG 12

AUG 


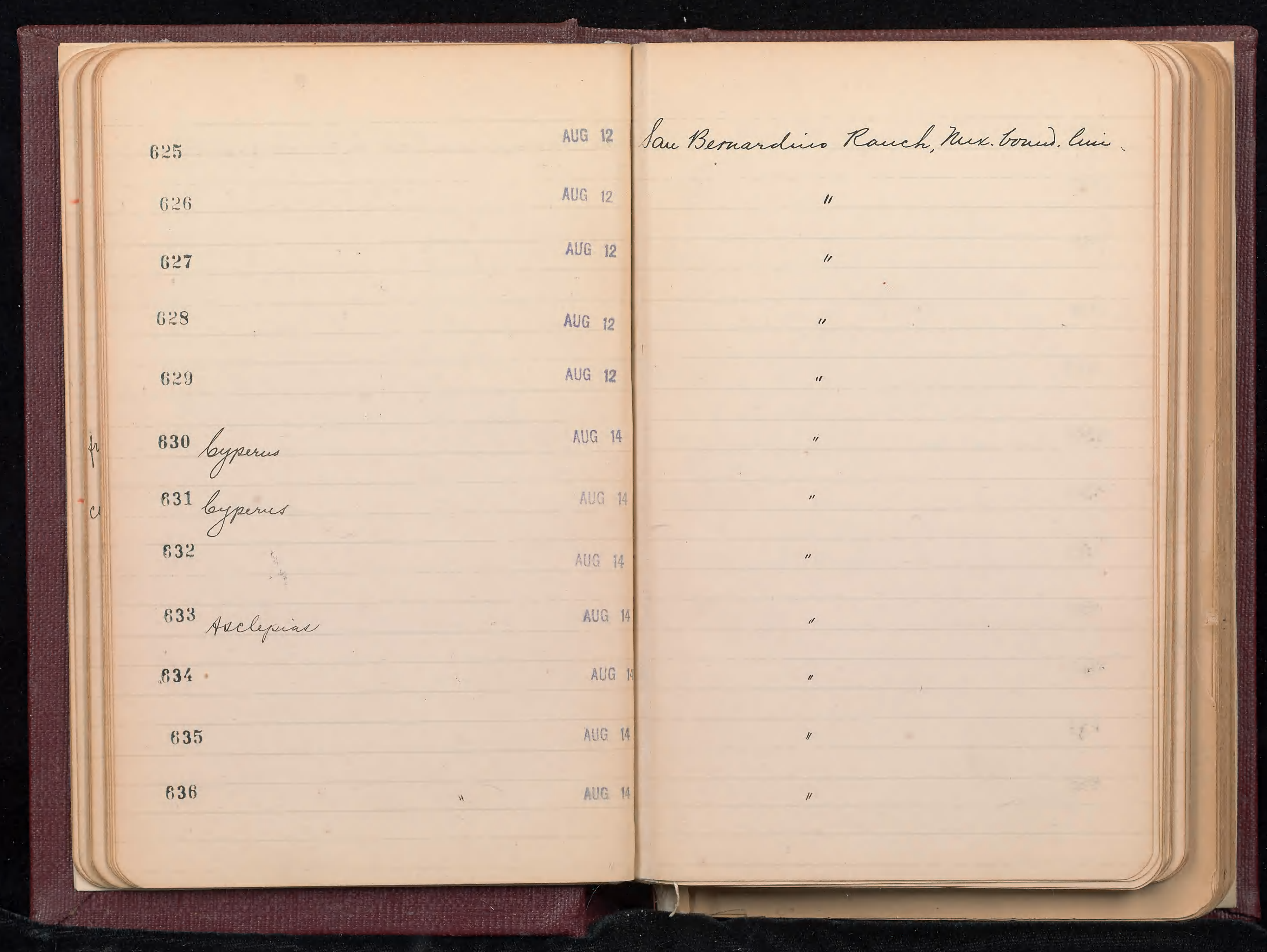




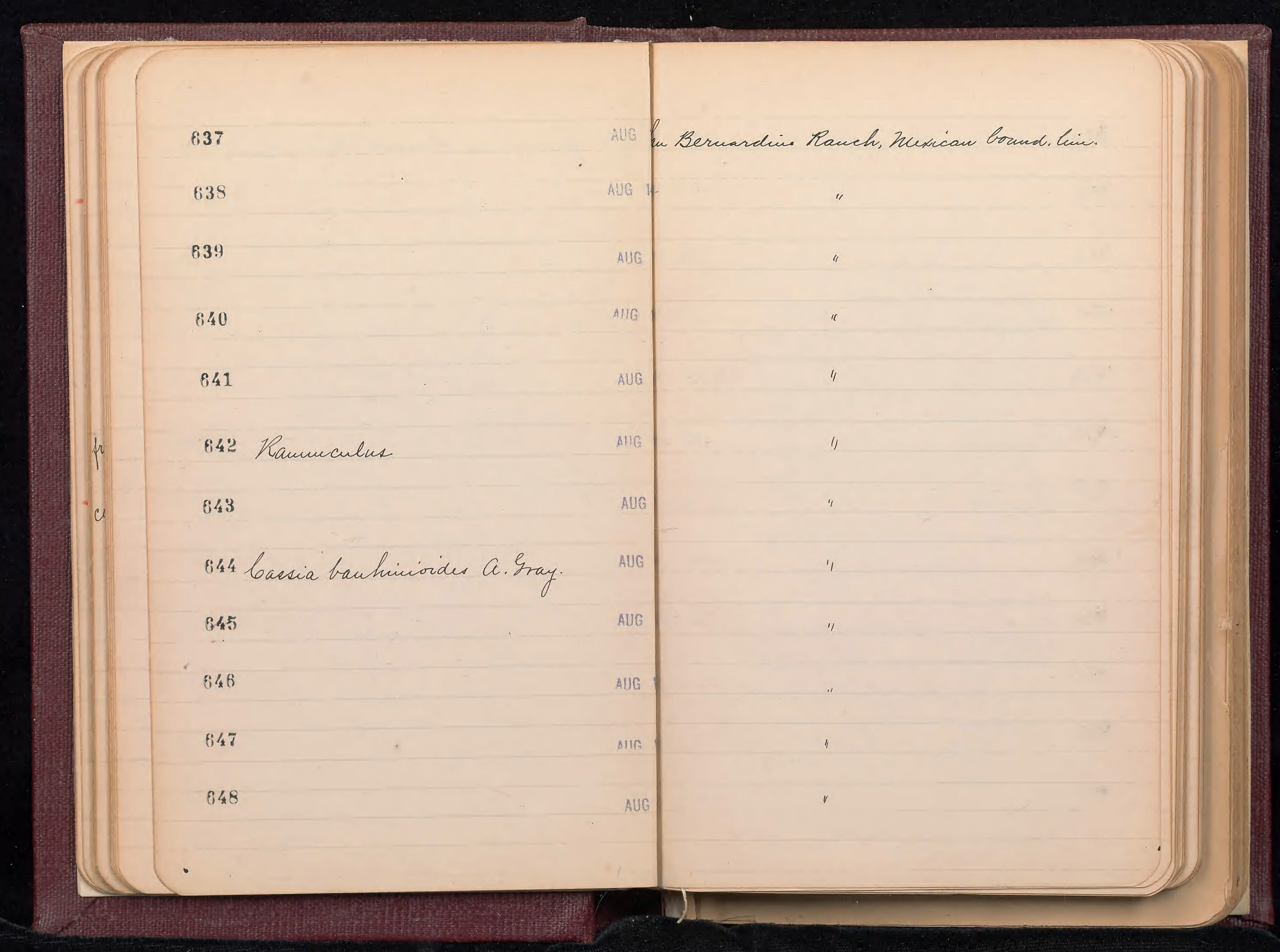


849

650

651

652 Cheuppodium

658

954 Polygonum

655 Physalis

856

857

658

859

860
AUG Saw Beruardies Rauch, Muxicau boundary lim

"

AlIG

AUG

(1)

$A \cup$

AUG

AUG

AUG

AUG 


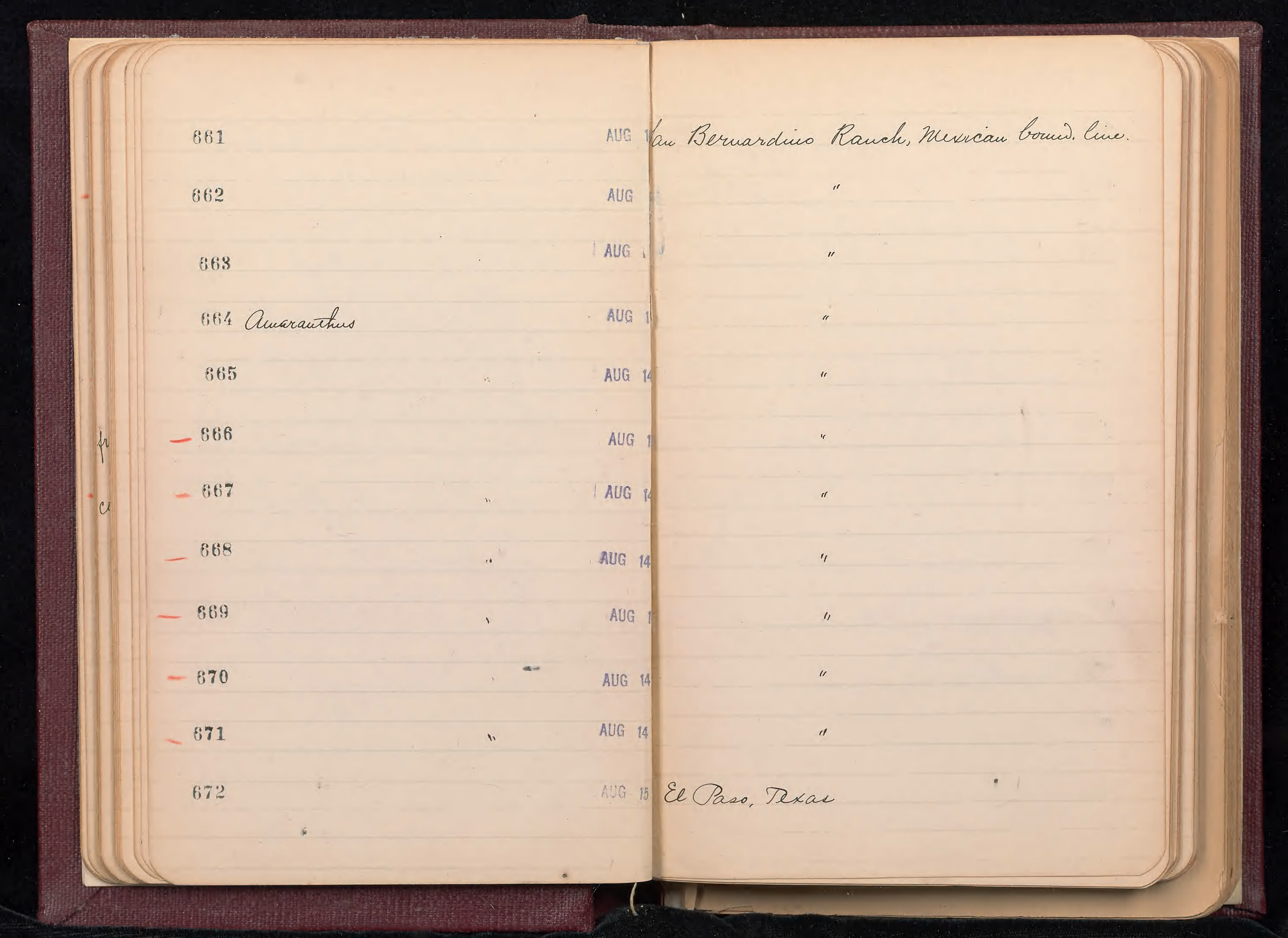




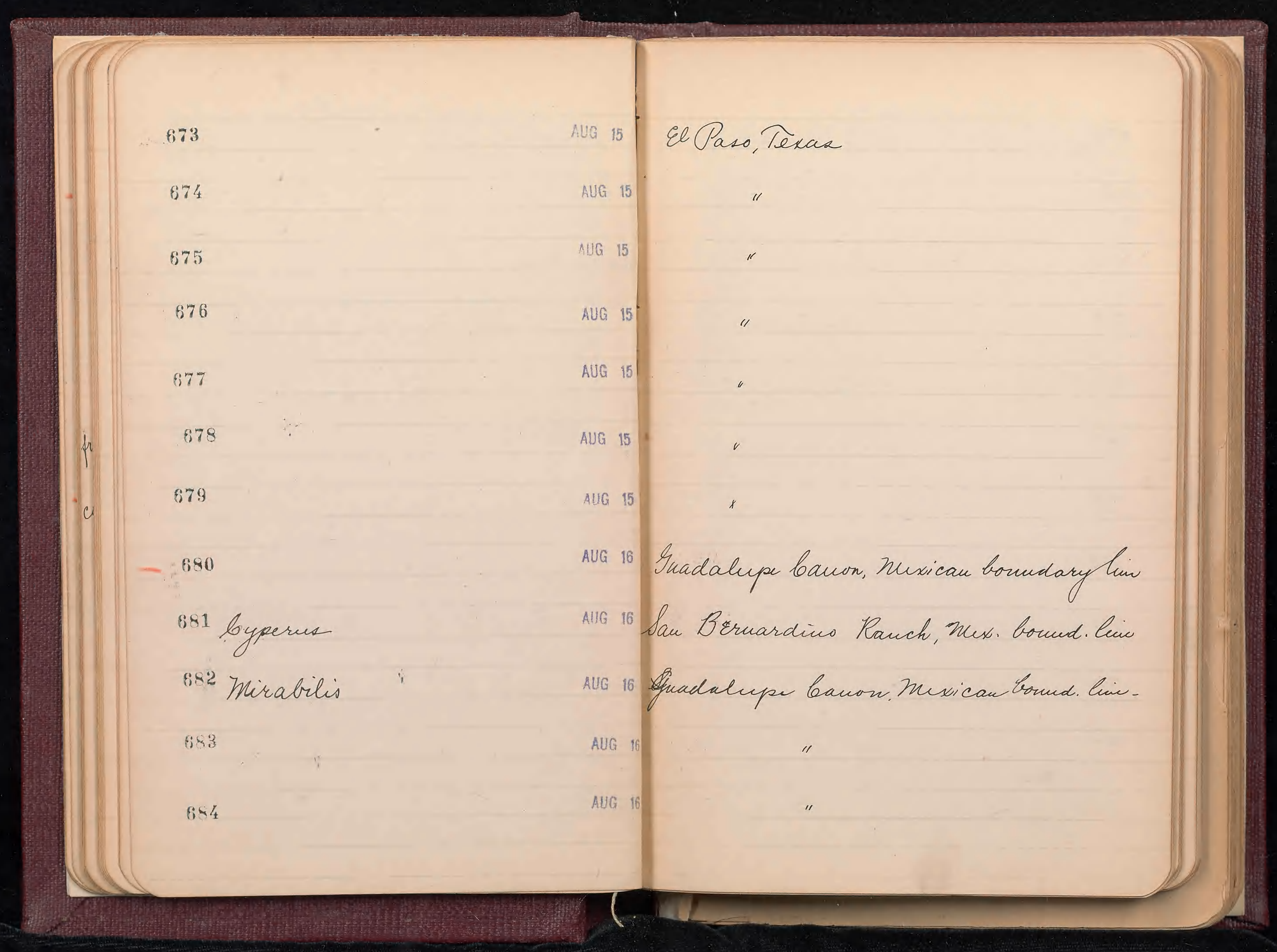




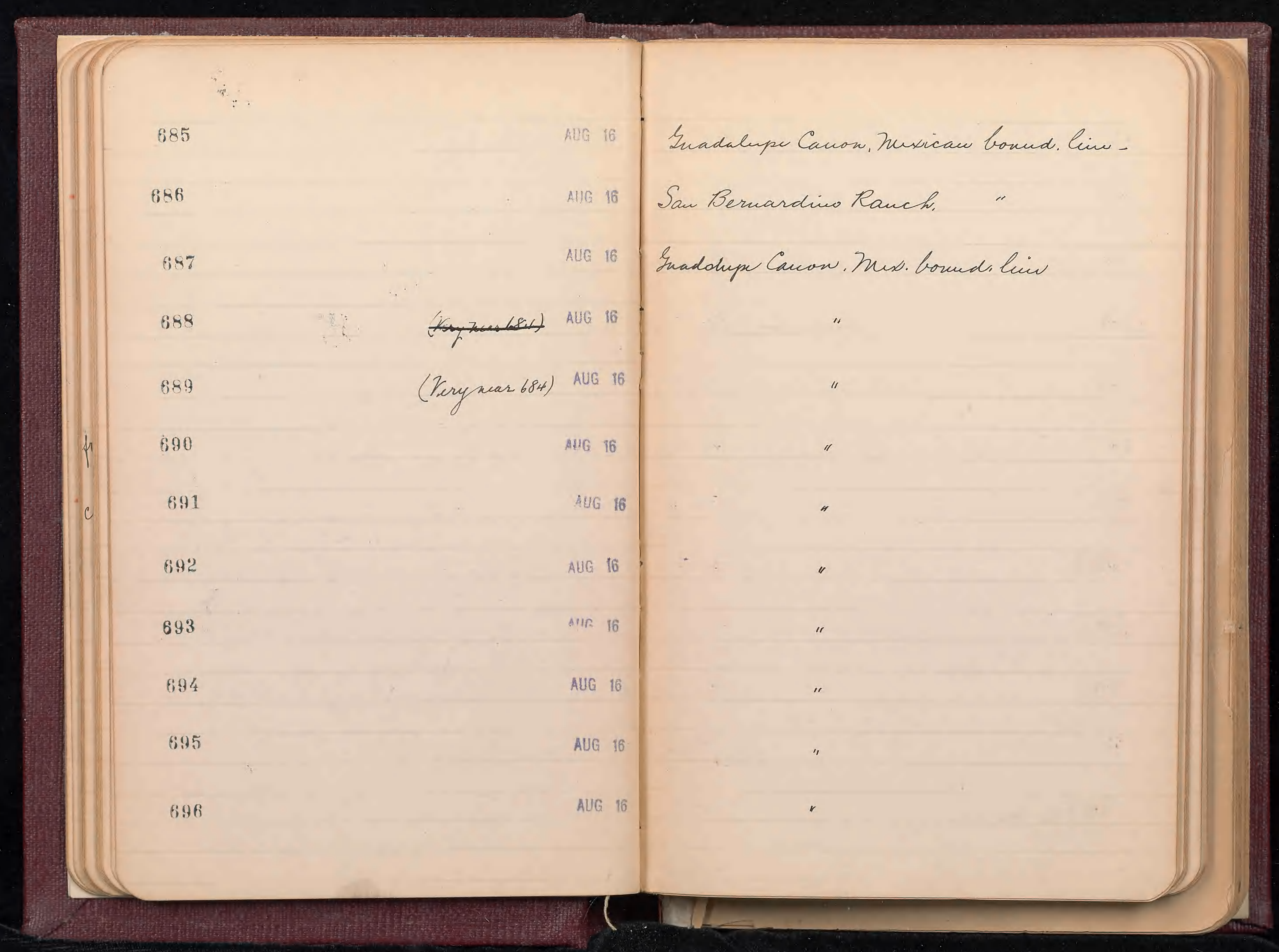




\section{7}

698

699 Yhue

700

701

702

703

804

1905

106

707

109 leypenus
Sundalupe bañon, Muxicau bonudary hiv

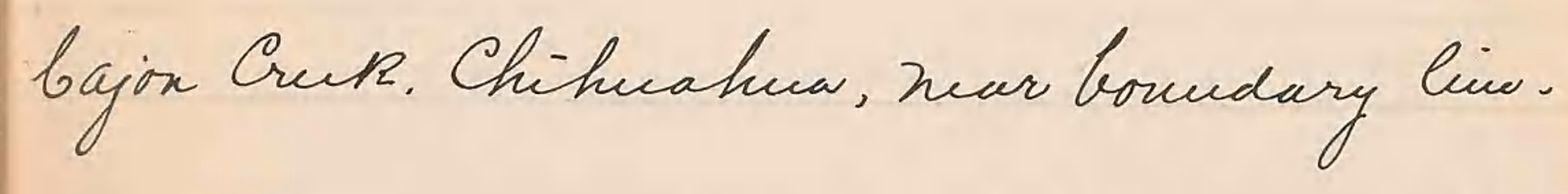
Snadalupu Canon, Musicaus bonudary liner.

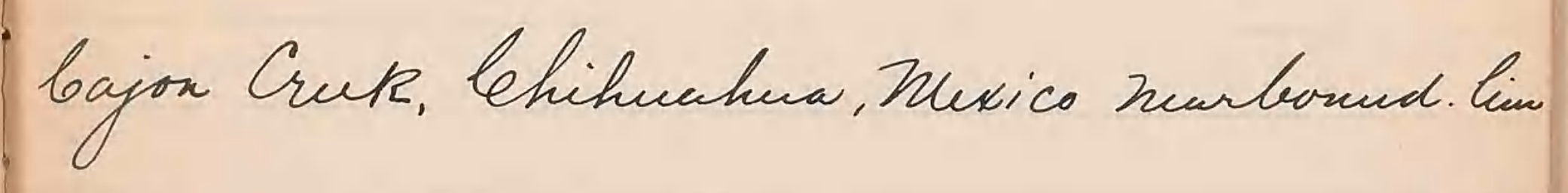

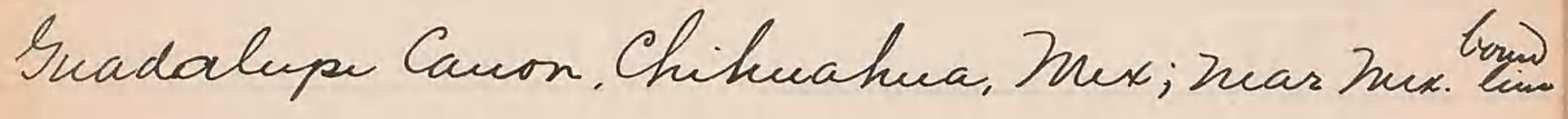
Sau Beruardino Rauch, Nue bonend. liue. 
:09. Lеурети

10 bassia Mishigeni S Sray. 911

912 Polygonum 813 Eriogonum 914

i. 13

216 Lyctrum

$81 \%$ Asclepias

915 bicuta maculata

919 Panicun

920
San Buruardius Pauch, Nex. bound. Bie.

AUG 19

AUG 19

AUG 19

AUG 19

AUG 19

AUG 19

AUG 19

AUG 19

AUG 19

AUG 20

AUG 20
"

11

or

“

"

ir

" 
\$21. Paspalum

722 Setaria

123 Asclyoias

724

725 loyperus

726 Cicuta maculata 2 .

727 Carsia leptocarpa Beuth.

925
AUG 20

AUG 20

AUG 20

AUG 20

AuG 20

AltG 20

AUG 20

AUG 20

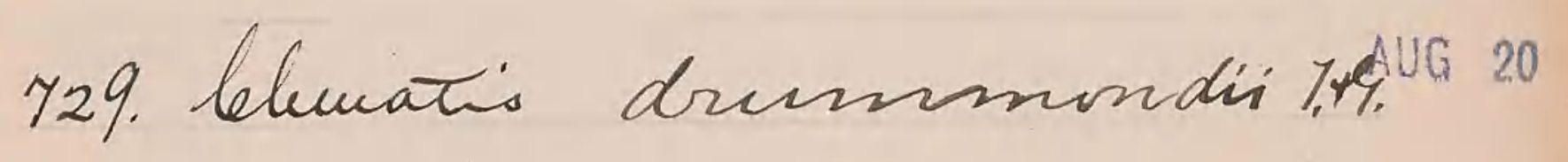

130 Prosapis

831 tnucus

932
AUG 20

AUG 20

AUO 20
Sau Beruardino Rauch, Mux. Bown. Cin.

/ 
733

734 Inentzelia

735 Salix

1836

737

735 Paricum

739

g10 Eragroatis

$\log 1$

192 Paspalum

1983 Panicum

984
AUG 20

San Beruardius Rauch. Hux. Bound. Lim
Aug 28

AUG 28

มีบิ 28

AUG 28

AUG 28

AUG 28

AUG 28

AUG 28

AUG: 28

AUG 28
II
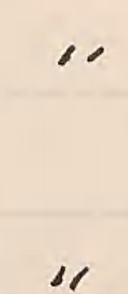

"

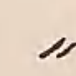

1
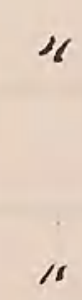

"

"

II 
745

$$
946
$$

898

748 Asclepias

199 leyperus

750 Cyperus

951 bascia leptocarpa Buth. AU: 28

752

953

1954 byperus

1955

856

AUG 28

Ang. 29

AUG 31

AUG 31

ำำ 3

AUG 31
AUG 28 San Beruardius Ranch, Mex. bound. Cin.

"I

I) 
$75 \%$

259

1759 Physalis

760 Lpomvea

961

1862

\%63

964 Andicago sation 2

ref5

766

$76 \%$

765
AUG 31

AUG 31

AUG 31

AUG 31

AUG 31

AUG 31

AUG $3 !$

AUG 31

AUG 31

syet 1

$\sin 5$

$\operatorname{ser}(5)$

SEP 5
San Beruardius Rauch. Mex. Gound. Cin.

"

"

$"$

"

"

,

bajon Bonito Greck, Sonrra, har hux. Cornd. Cice San Bernardins Pauch, Hex. borue tien. y 


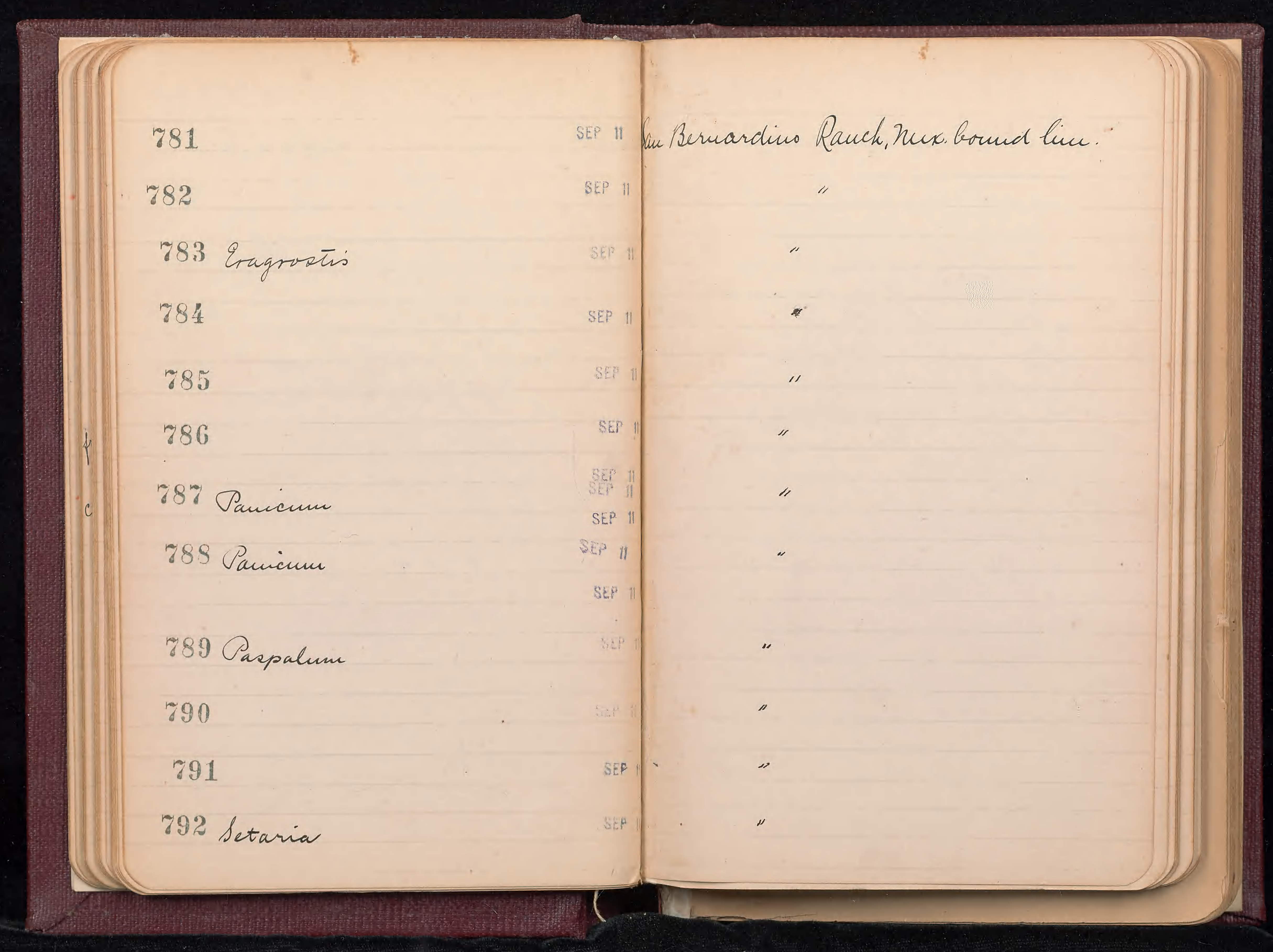


793

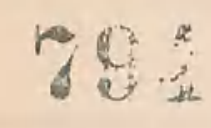

955

896

997 byporus

99 Cyperus

1999 Euthamia

800. Cleunatis drummondis T.H. EP

801

802

803

804 aster

SEP

SEF

SED

SEP

SFP

350
SiP 11 San Bernardius Rauch, Nux. Bormal. Riur.

SEF If

SEI

SEP

$"$

Sep II
1

"

" 
805

906 Solanim

$80 \%$ pomvea

805

809 Amaranthus

810 Asmaranchus

811.

81\% Lepichime Thurberi Znotis.

813 Eleochario

814 Elecharis

815

S16.

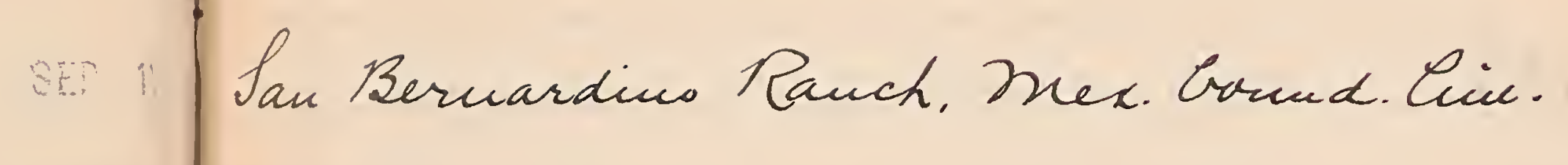

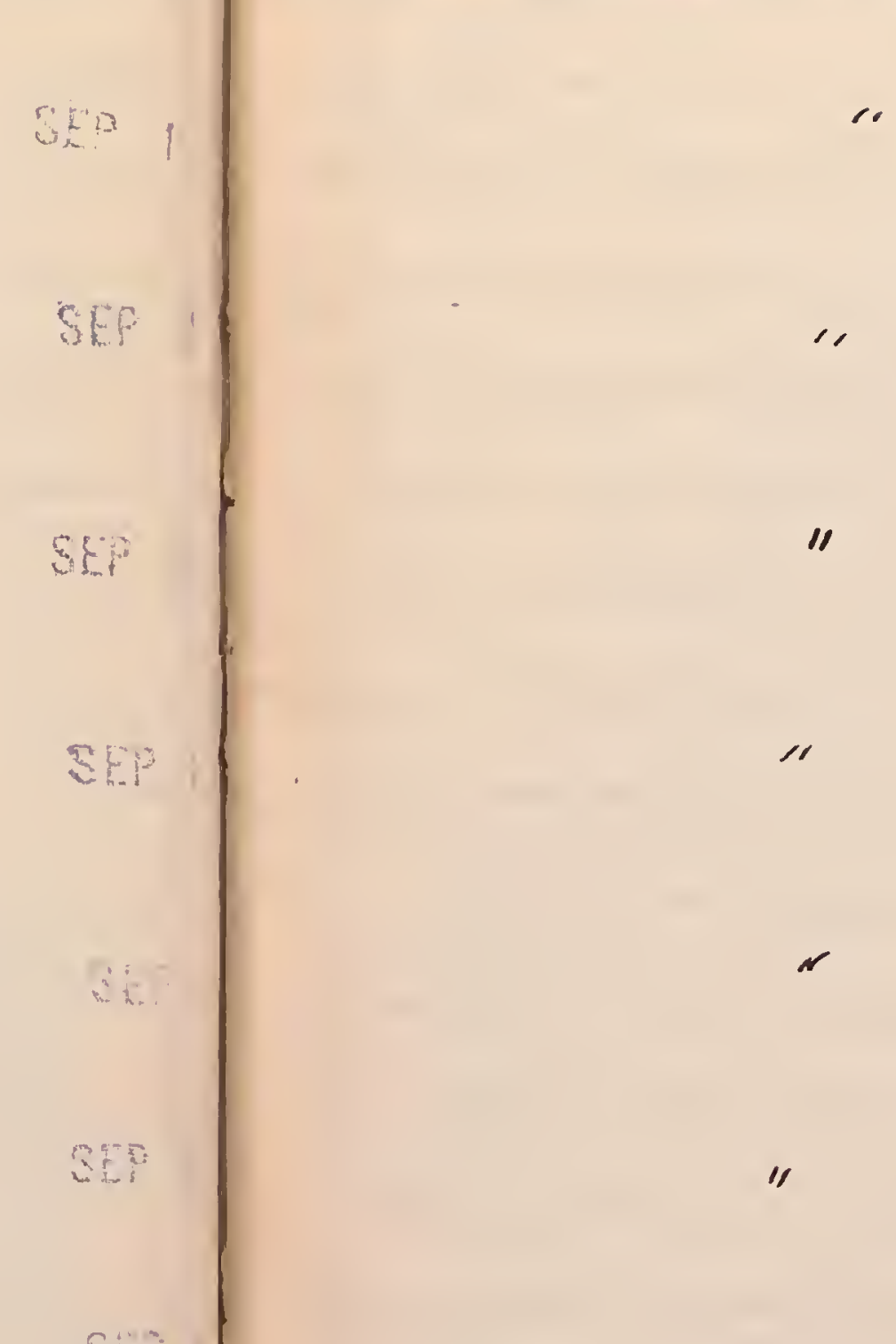




\section{$81 \%$}

819

819

820

821

82\% Lobelia sptendure

823

8\%: Ribes

825 Saura

826 Eyphorbia

$82 \%$ Mentzilia

82S Xauthinu

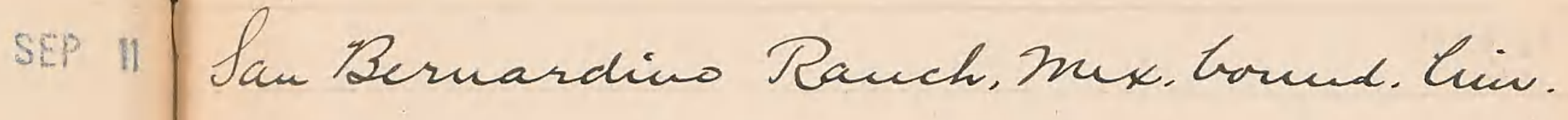


829 Hydrocotgle

830

831

832 bonundina

833

834

835

83

$43 \%$

839

839

840

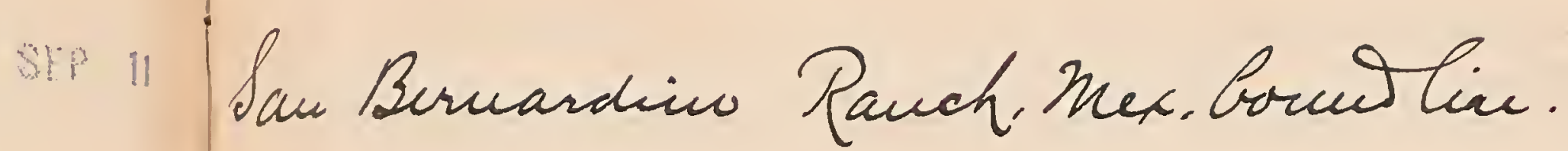

$\cos 11$

St) 11

$6 \sin ^{11}$

SE 11

SFP 12 Pedrogoza nto, nex. bound. Cine-
5612

seft 12

$\rightarrow \infty 12$

$8)^{2}+12$

(2)

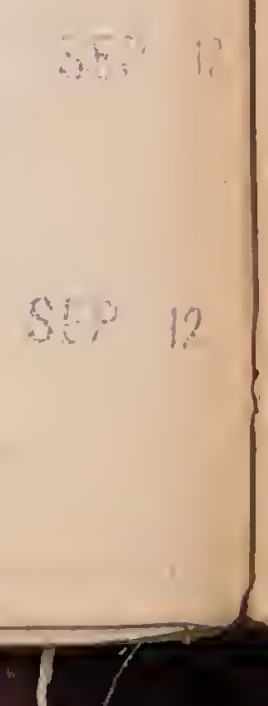


81

842

843

818 Acacia

815 Cassin Tislizani A lray

816 fucurbita palmato

84

814

849

850 Errogrnum

851

852
SEP 12 Pedragosa 3nts, mex.bruid. Ciue

SEP 12

SEP 12

12

SEP 12

50

$6 t^{3}$

SEP



SEE

SEP 12

SEP 12

स 12

12 1 
853 Mocrhavia

854

855

856

$85 \%$

859

859

860

861

862

863

864
12 Pedrogosa mis, mex. bound. live.

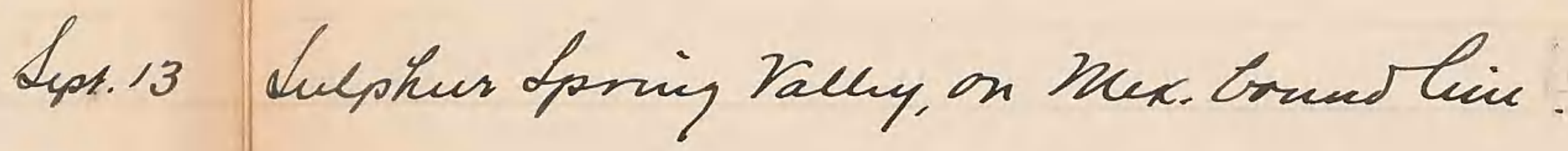
5EP 14 Mexican torudary hiu, S. of Bisbu, Ariz. SEI IS
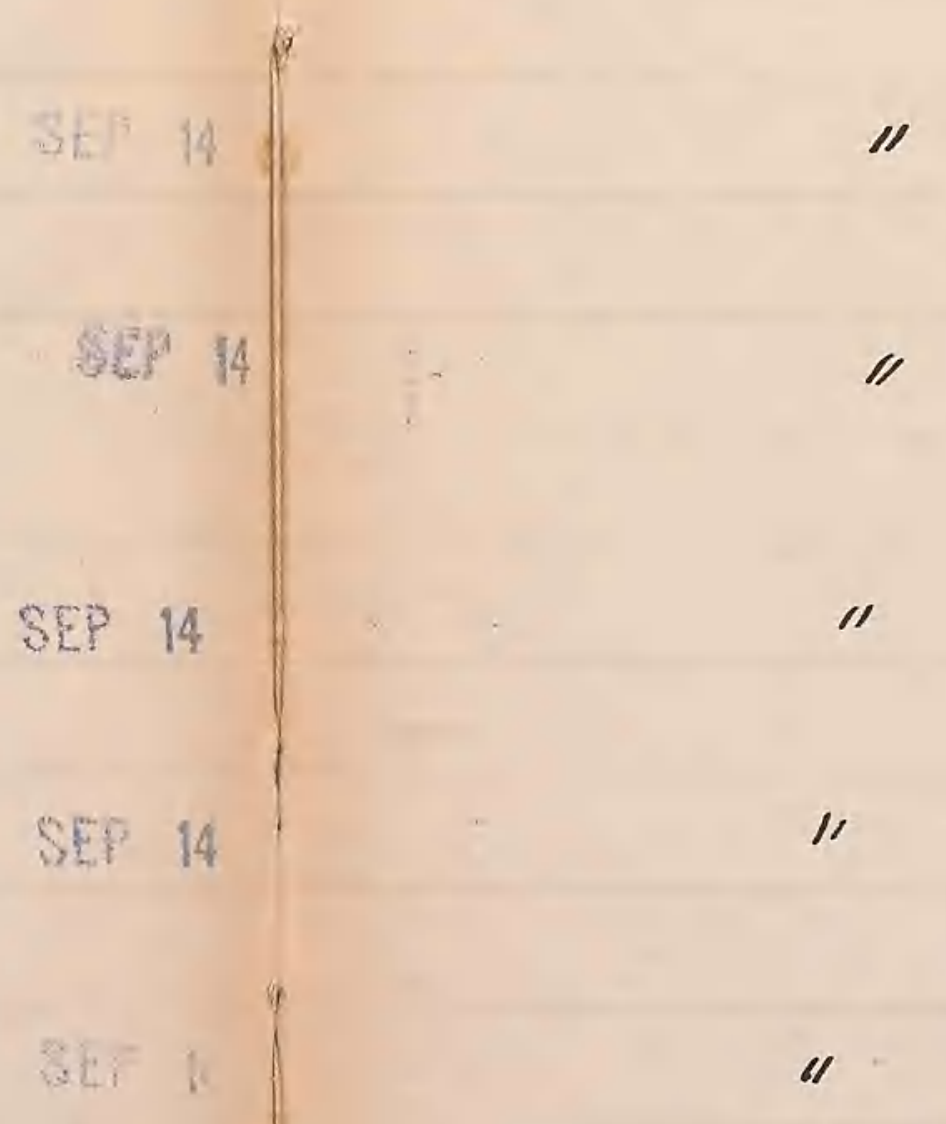

SEP

gets is

SEP 14

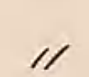




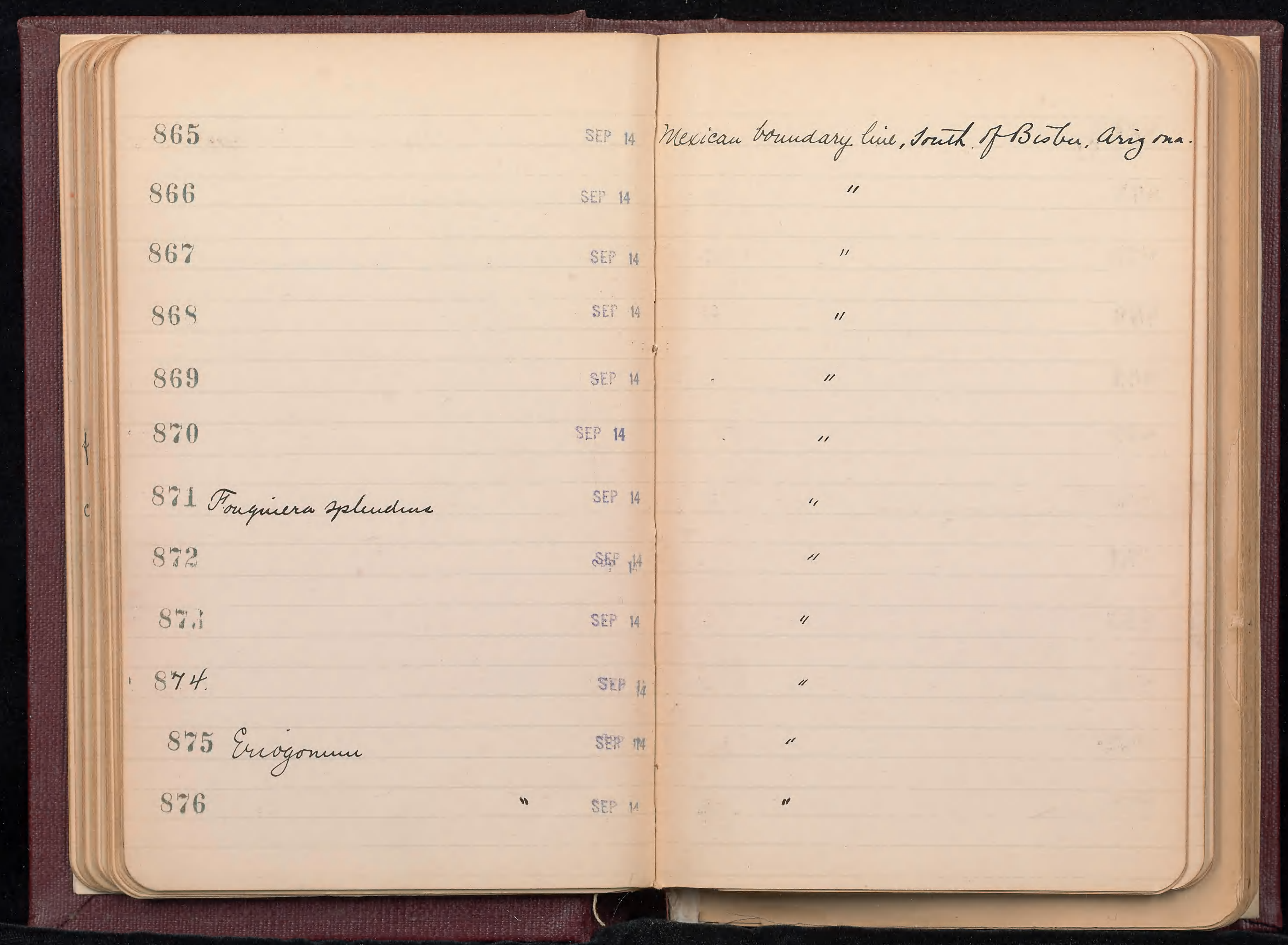




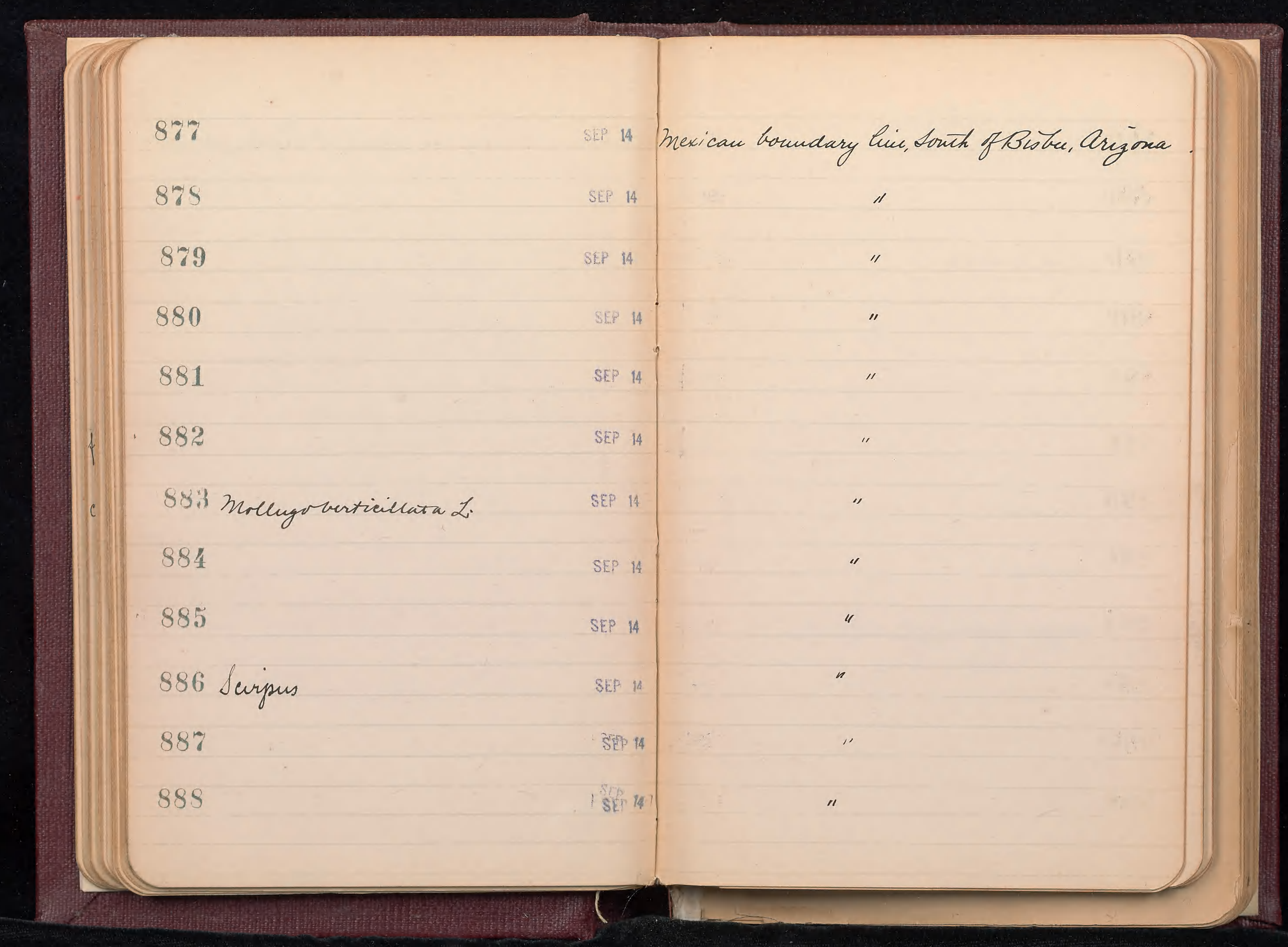


891 Phoradcudron

892

893

894

895 Lolausurat

890. Ceyperus

$899^{\circ}$ bastia leptraysa Buct.

89.9

899

900

SEP 14

set 14

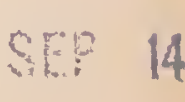

Se: 14

$8 \mathrm{se}$

of 14

SE? 14

SEP 14

SEP

8. 14

set 14
Inexican bonndury him, Aonth Histuen, Ariz.

"I

11

"I

,

is

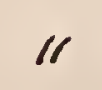

$1 /$

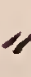

" 


\section{Explorbia}

902 Exphorbia

903 Erigiron

904

905

906

$90 \%$

90 S Arouia

909 Oenothera

910 Exiogonum

911

912
SEP 14

Wexican bound. Hies Rowth of Biobu, Aripona

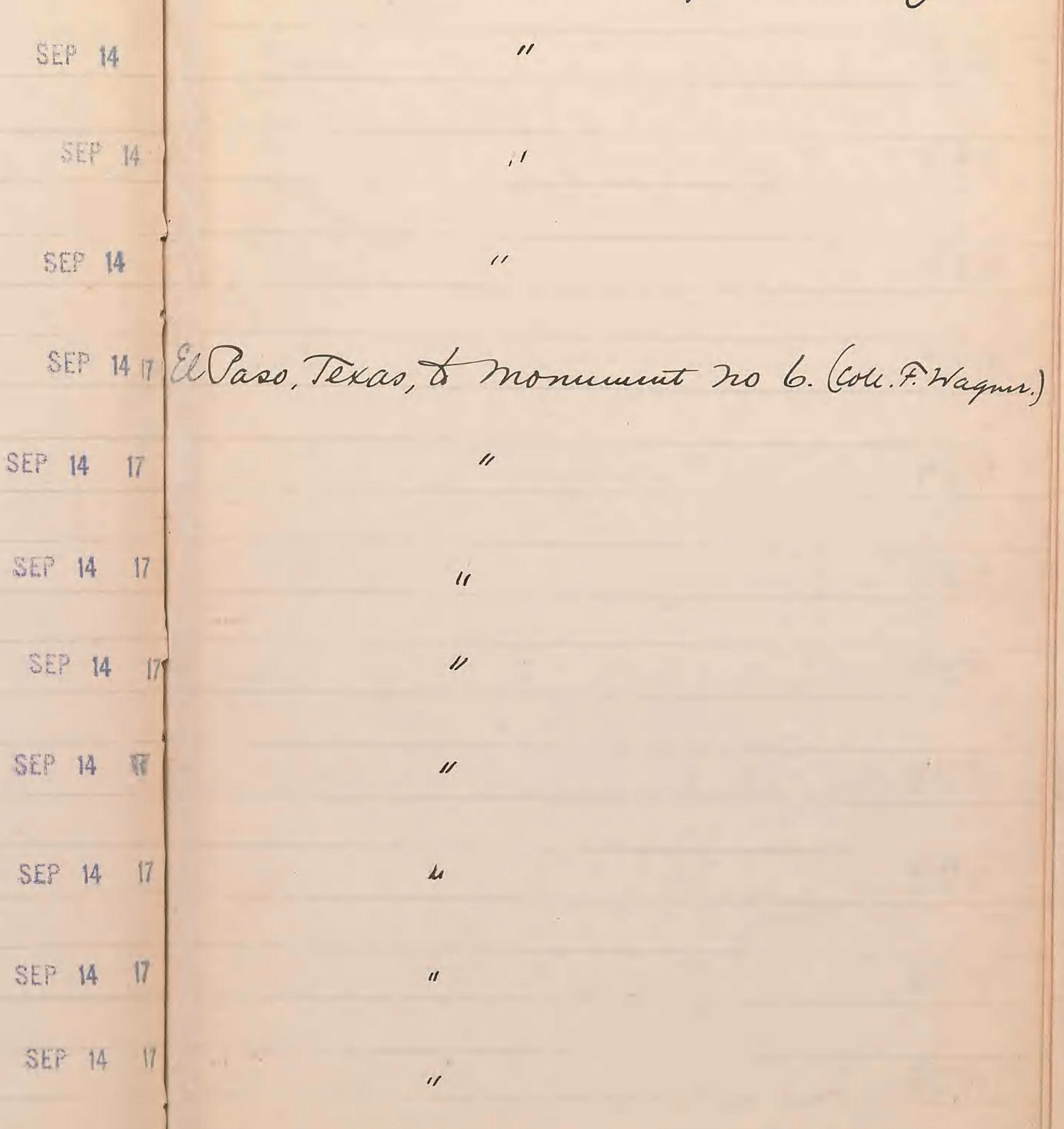


914 Lefidium alyssoides ahriy it

915

SEP 14

916

918

915

919 Źminia

920

921. Proelichia

922

923

924 SE? 19

stop 19
SEP 14

SEP 14

SEP 14

SEP 14

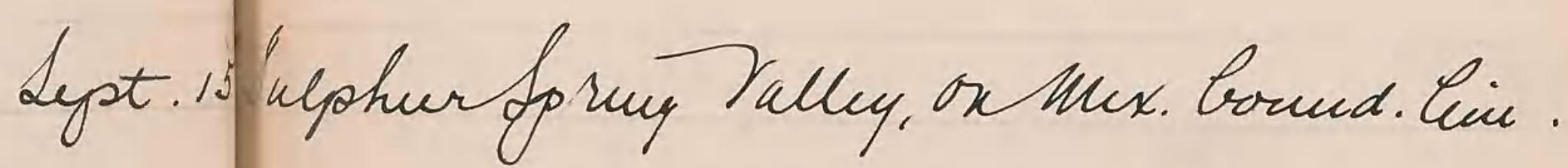

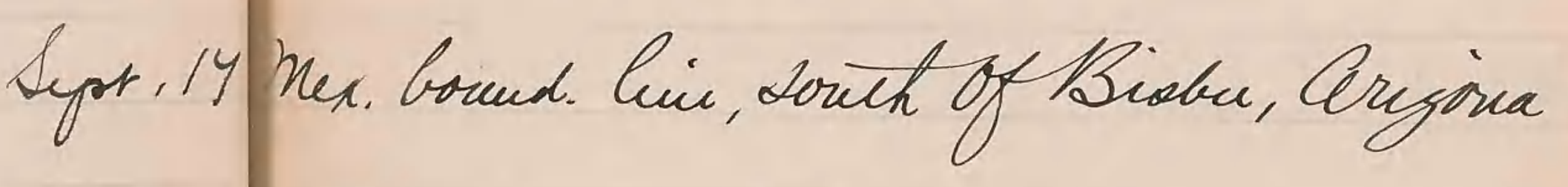

nx

/l

/1

II

11 
$93 \%$

935

939

910 Pronaca

$9 * 1$

912. Argenone

913

911 hatlymia proboscidea

915

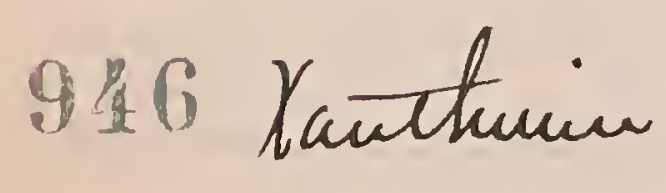

918 Pectis

919 Cucurlita digitata G. bran
Hexican boundary line, Louch of Bibu, Arizona.

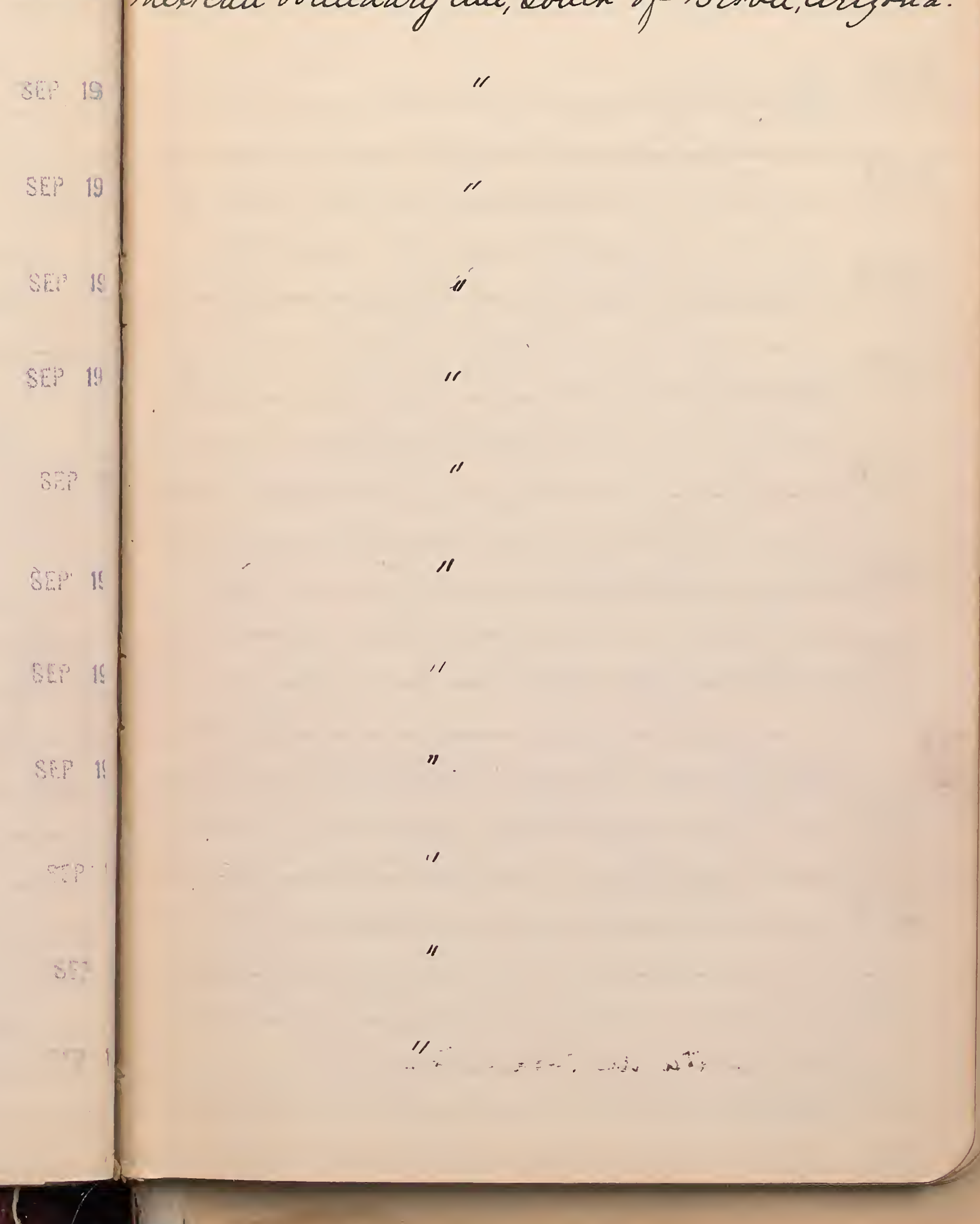


949

950 promoen

951

952 Leijas

953 ponvea

954

955 lovngz a Coulteri

956 fimin

958

955

959

960

961 Aptopappons

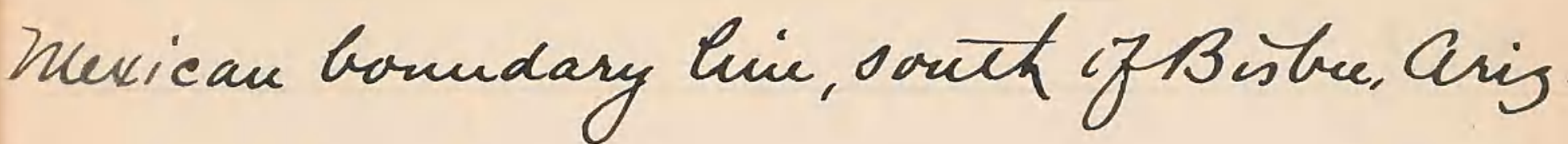
SEP 19

SEP 19

Stp 19

SEP 19

SEP 19

SEP 19

SEP $20 \quad 24$

Moncunt ho 6 to hox. no/2 (L. Nagmara)

SEP 20

SEP 20 2 2

SEP 21 स Tombotone, Arjona 
962

963

964 Pris

965

966

967

968

969 bicuta

$9 \% 0$ Pecti:

$9 \% 1$

922

973 Euphorkin
Lypt.26 San fosi nets, Sonora nux.

"I

Lept 30

" Bisker, Arizona.

Lept. El Paso, Tex" to homenunt no $\sqrt{3}$. (7. Waymer)

"

“

16
"

If

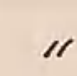


$9 \% 4$ Verbeiva

$$
985
$$

$9 \% 6$

$9 \%$

$9 \% 5$ bontlea tridentata

999 Solaumm

980

981 bevallia

982 Nicotiána

983 Ocuothira

984 Eyphortina

985

Lypt. El Paso. Tex., to hoomunt no 53. (F Waynu coll)

11

《

"1

"

"

"

/

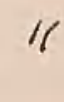

"I

4

ic
,

"

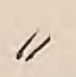

4 
986

$98 \%$

989

989

990

991 Eriogrumm

992 Malvacopeis

993

994

995 Pecti

996

997

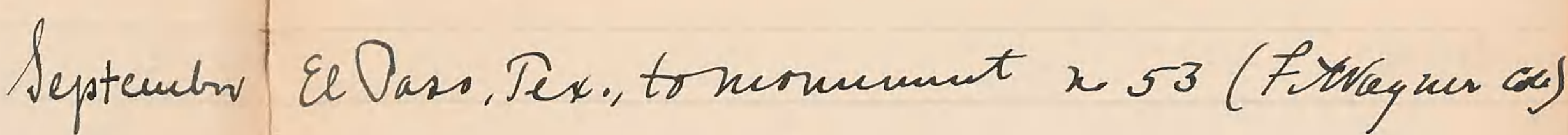

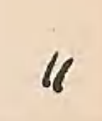

,

II

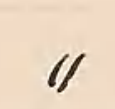

II

"I

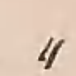

OCT I

San fose' mot, Lonora, Hex. 


\begin{tabular}{|c|c|c|}
\hline 999 & OCT 1 & San foei Hto, Sonora, Hex. \\
\hline 999 & OCT 1 & "I \\
\hline 1000 & OCT I & "I \\
\hline 1001 Querens & OCT 1 & " \\
\hline 1002 & OCT 1 & "I \\
\hline 1003 Henchera & OCT & " \\
\hline 1004 & OCT 1 & " \\
\hline 1005 & Oerz & Monumut to 18. (F. Wagnen coll.) \\
\hline 1006 & $\mathrm{Cer}_{2}$ & Hex. bount. leir, sauch of Bis bec, anj \\
\hline $100 \%$ & OCT 3 & Brom, ariz \\
\hline 1009 & OCT 3 & " \\
\hline 1009 & & " \\
\hline
\end{tabular}


1010 bassia Snichemicrimualchule OCT 3 Bibu, Arizona

1011 Brickellia

1012

1013

1014

1015

1016

1018

1015

1019

1020

1021

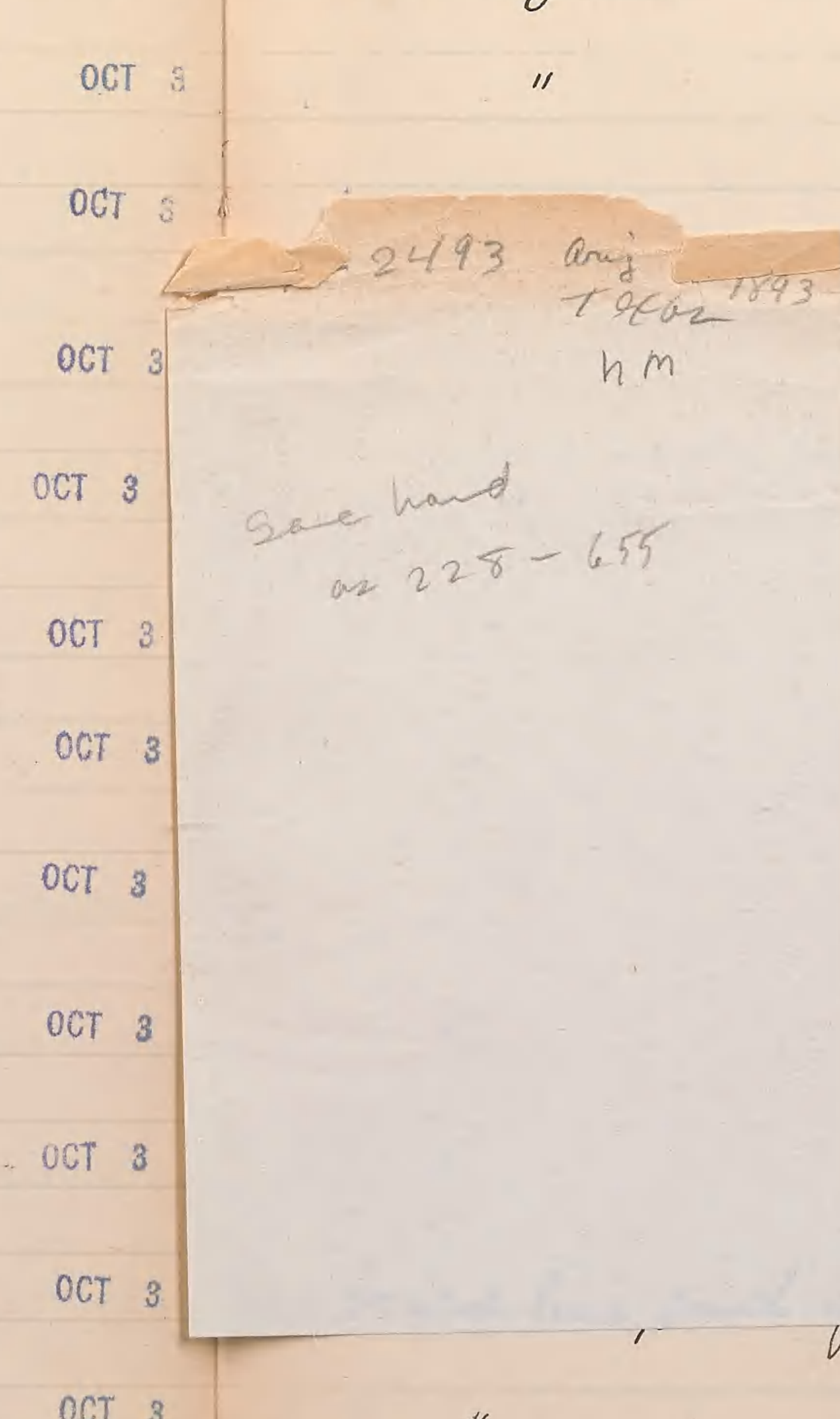
Wistue Ariz. 
$q-2493$ arisitipgz h $m$

Soce hand as $228-655$ 
1010 barsia Lnichemierimua Schule 1011 Brickellia

1012

1013

1014

1015

1016

$101 \%$

1015

1019

1020

1021
OCT 3

Bisbu, Aryona

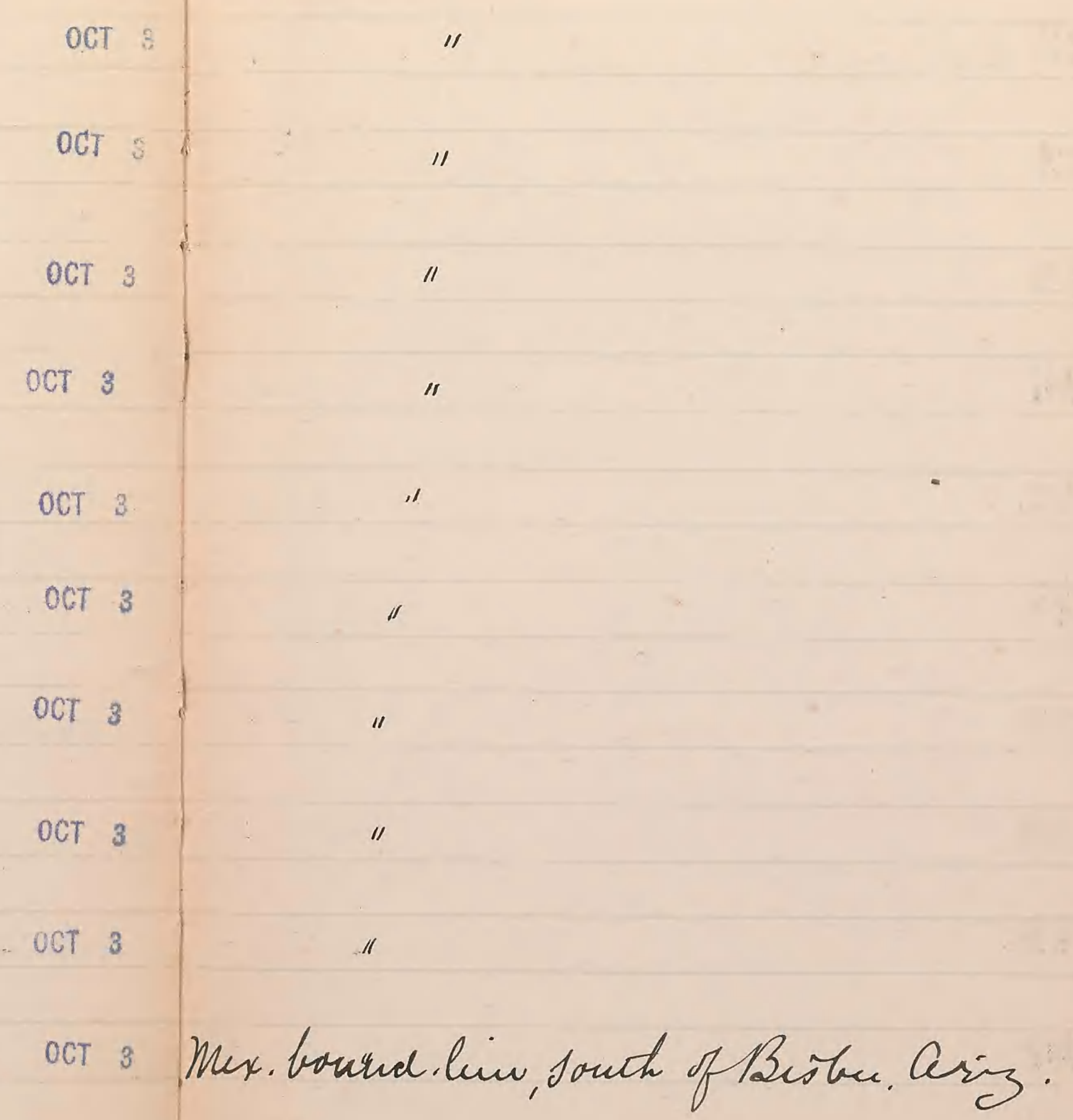




\section{2 \\ 1023 Artenisia}

1024

1025

1026

$102 \%$

1029

1029

1030

1031

1032

1033
OCT 3

OCT 3

OCT 3.

OCT 5

OCT 5

OCT 5

OCT 5

OCT 5

OCT 5
Wex. bound. Ain, douth of Bisbu, Ariz.

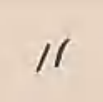

II

"I

11 


\section{4}

1035

1036

1037

1039 Lefidium Thurteri Hnotm OCT : 1039

1010

1011

1012 Jatropha 1013 Euphorbia

1044 Eypatorimu 1045
OCT 5

OCT 5

OCT 5

OCT 6

OCT 6

OCT 6

OCT 6

ocT 6

OCT 6

OCT $\&$

OCT 6 mex. bound. hiw south of Bisku, Ainz -

$$
\text { "I }
$$

" *

"

$\rightarrow$

,

San fosé huts, Sonora, Nex.

Mex. bounolim, souct of To iskes, Aring "I

San fosé heto. Sonora. Next. 


\section{6}

101: Rhus

1045 funjurm pachyphitace Torr.

1019 Juglans nupestris

1050

1051

105\% Rhus radicaus L

1053 Vitis

1058 Enogonum

1055 Artunicia

1056

105: Robinia
uCT 6

OCT 6

OCT 6

OCT 6

UCT 6

OCT 6

OCT 6

OCT B

OCT 6

OCT 6

OCT

OCT 6
San José Huts, Sonora, thex.

Mex. bound. Kine, aonch of Bisber, ArizIan fose' hits, Sonora, hex. 


\section{5}

1059 Populus

1060 Erigeron Canadensis $\mathcal{L}$

1061 Cister

$106:$ Eniocarpum

1063

1064

1065

1066 Eragrostis

$106 \%$

1065

1069
UCT 6

OCT

OCT 6

oct 8 .

$\cos 8$

OCT 9

OCT 9

OCT 9

OCT 9

OCT 9

OCT 9

OCT 9
San Gosi huts, Sonora, Muxico.

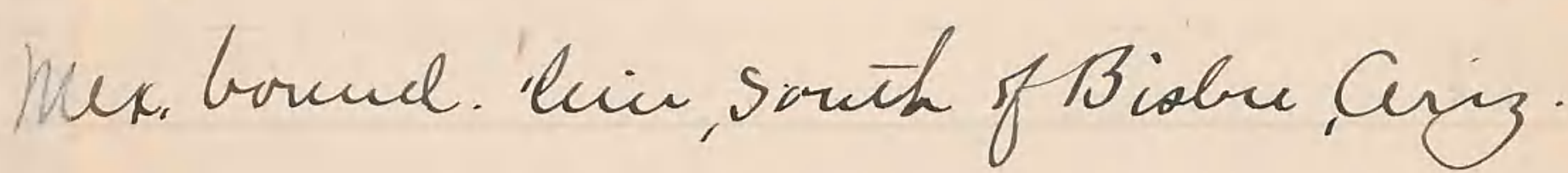

Putomas, nexx nar nion. no 2z. (7. Wagme) Bistu, Arizona.

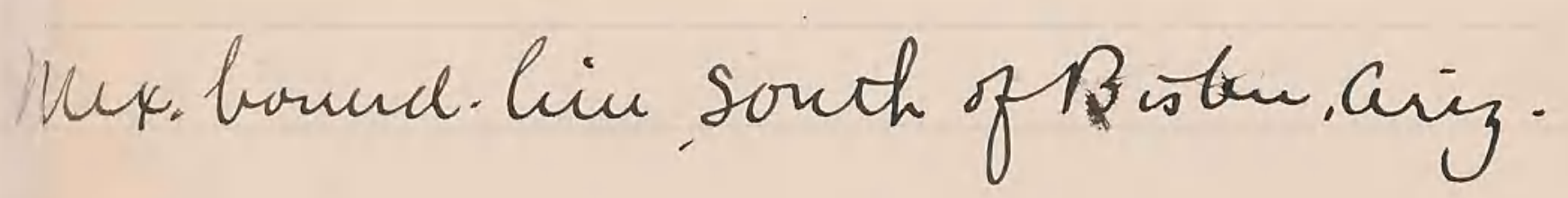


$10 \% 0$

$10 \% 1$ Eragrostis

10.2 Pancinn

$10 \% 3$ Eriogonum

10 's Mitrac aysus

$10 \% 5$

$10 \% 6$ Eyphorbia

$10 \%$ Mollugonenticillat a $\mathcal{L}$

$10 \% 9$ Dponoca

10:9 Priloria

1080 Hymunothris Wislizui C. Iry 1081 Prosoping julifara
Arin-

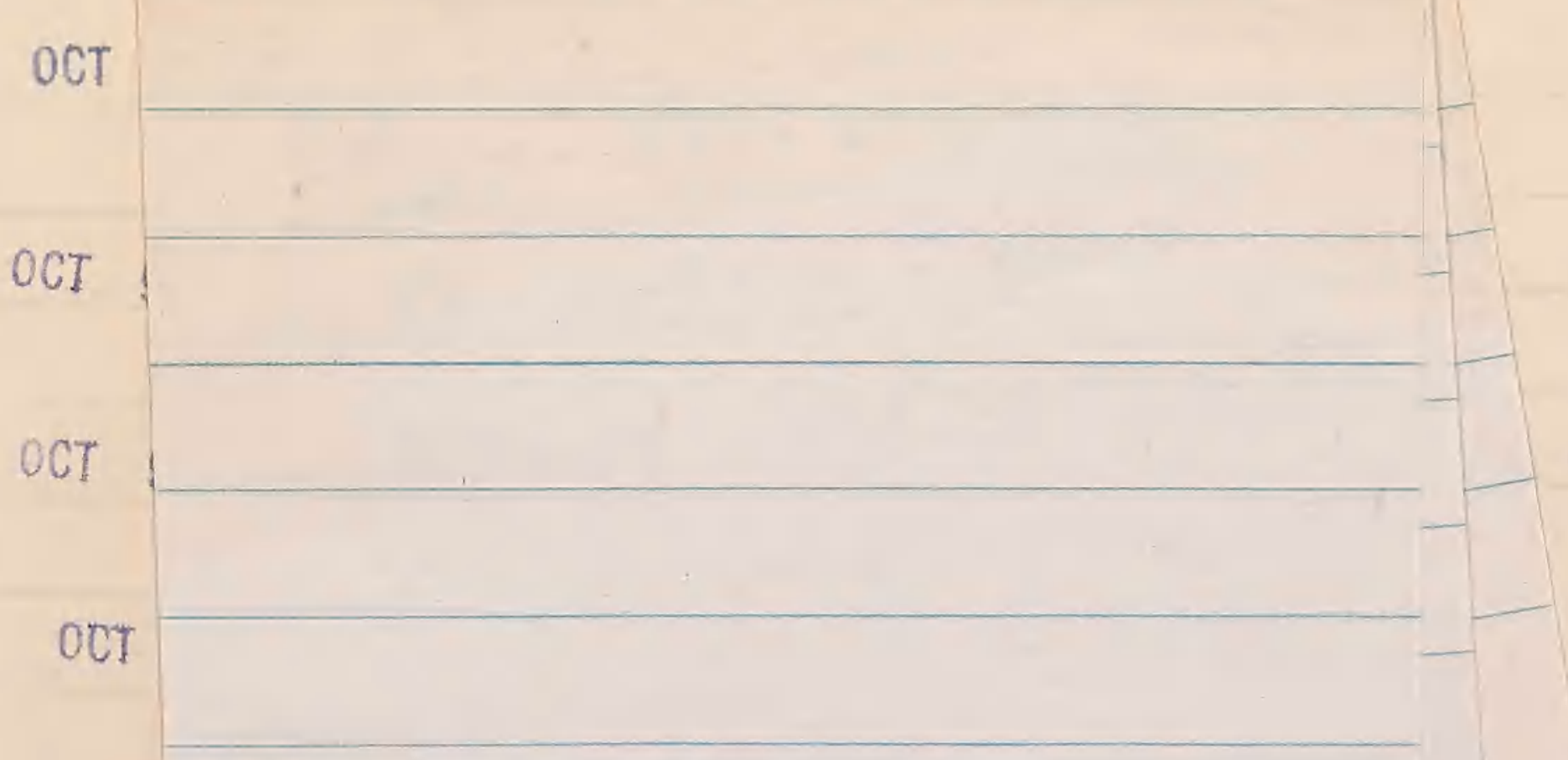

OCT

COT

OCT

OCT

oct

OCT

OCT 9

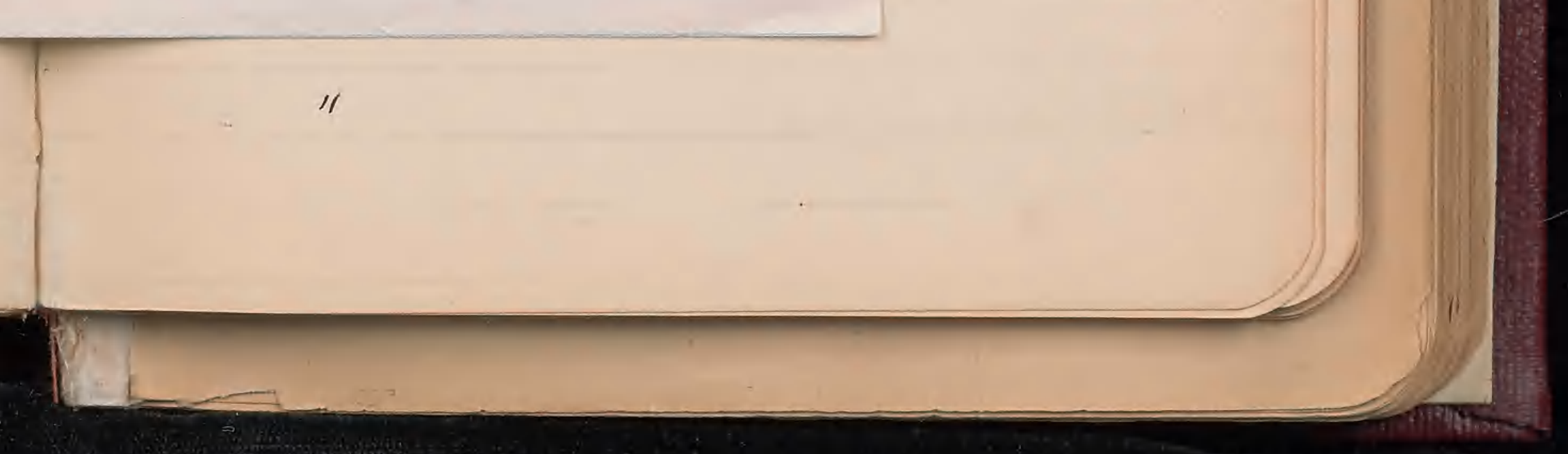




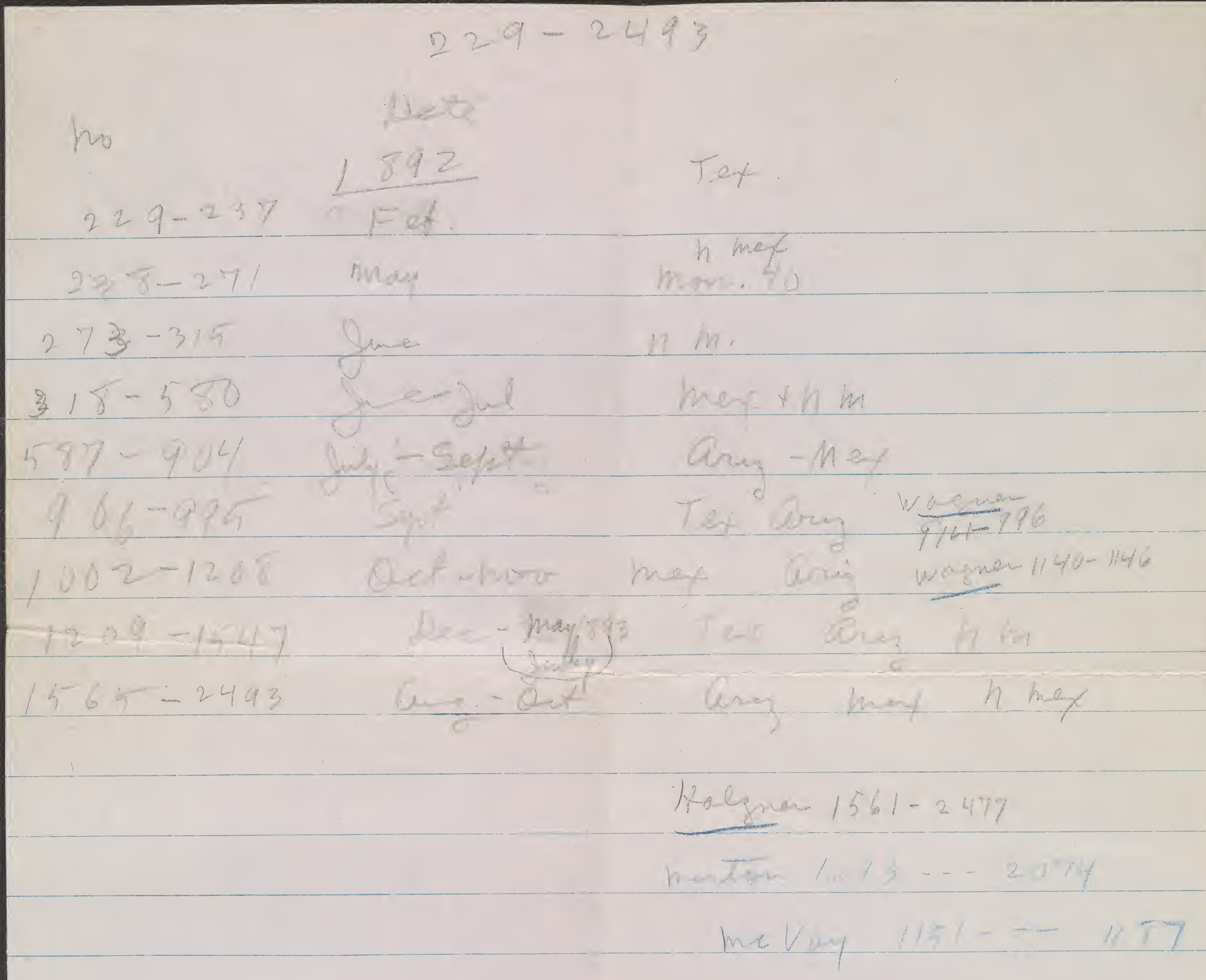




\section{$10 \% 0$}

$10 \% 1$ Eragrostis

$10 \% 2$ Panicinn

$10 \% 3$ Eriogorum

$10 \% 4$ mitrac aryens

$10 \% 5$

10:6 Eyphorbia

$10 \% 8$ Mollugonerticillat a $\mathcal{L}$.

$10 \% 5$ Dponoca

$10 \% 9$ Priloria

1080 Hymunothri Wislizui C. Irn 1081. Prosopin julifura
OCT 9

OCT 9

OCT 9

OCT 9

OCT 9

COT 9

OCT 9

oet 9

oct 9

OCT 9

OCT 9
Mex. bounid. him, Donth of Bibu, Ariz-

"

"

br

n
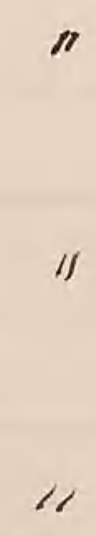

,

" 
$1108 \%$

1083 Esphorlia

11081 Joonoca

1085 Eriogonum

1086 Enigion

$108 \%$ Ciristolachica

1089 Hymuocha

1089 Colevanthus

1090 Syminolomia nulbiflora

1091 Yosspianthm

1092

1093 Aeculypha mex.bound. lim, South of Bisbu, Arig.

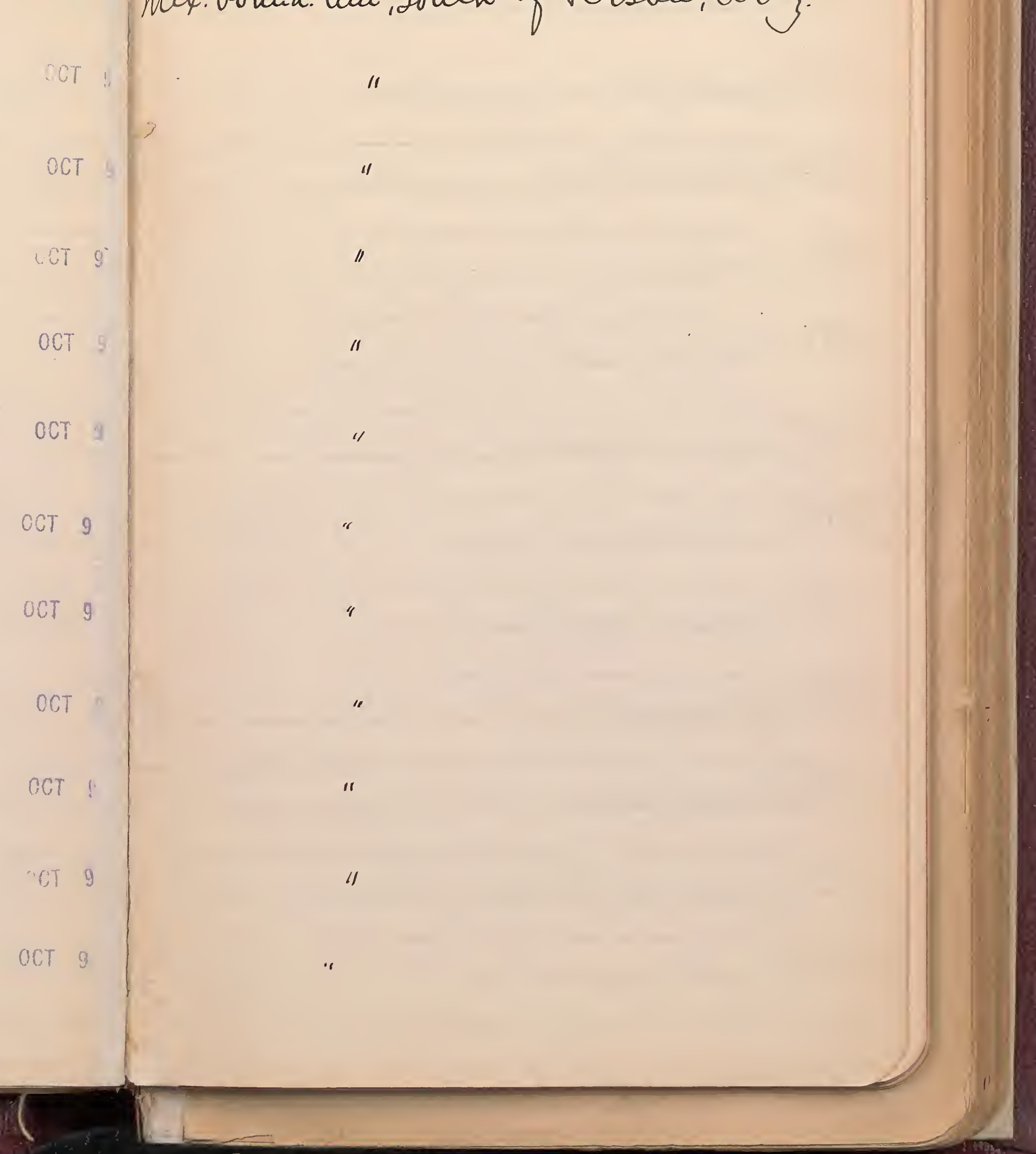




\section{Sida}

1095 Anbrosia

\section{Qmaranithms}

$109 \%$ Cettio

1099 Eriveargum

1099 Juglans rupistur

1100 barduns

1101 Guglaus Mupestris

1102 Yymuocha

1103 Bacchario

1104 Enpatorium Lreggii A Iray

1105 Aster

OOT \& Nex.bound. hin, South of Bistu, Ariz

\section{DCT 9 \\ DCT 9}

OCT 9

OCT

OCT 9

Oar.10.

$\mathrm{OCT} \quad 12$

OGT 12

OCT

OCT

nCT
Sam Pedro nivr Nuxu. bound lim

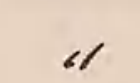

"I 
1106 Erio-caspum 110 ' Ater spmosus Buth.

1109 Datura neteloides DC.

1109 Lolidago

1110 Salix nigra 2.

111 Salix

1112 Oxalis coniculata L.

1113 aster

1114 Solidago

1115 Aster

1116 Yerbena

111 Anbrosia
Oor 12 San Pedro Revir, Hux, bound line -

$\cot 12$

007

OCT 12

ont 12

OCT

OCT

OCT : 
1119

1119 iragrostis

1120 Parienm

1121

1122

1123

1124

1125

1126 Eragrostis

$112 \%$

1125

1129 Cheoris
OCT 12

San Pedro Skivr, Mnxican bonudary him.

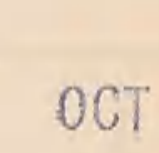

OCT

OCT 12

OCT 12

OCT

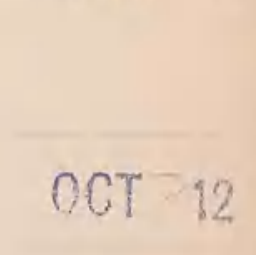

ocr 12

OCT 12

OCT 12

OCT 12

तCT 12

OCT 12

OCT 12
"

“

,

"

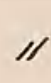

"I

"I

"I

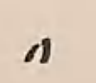

"s 
1130 Panienm

1131 Lossypianthus lanugniosus (Poir) mogy OCT

1132 Hymuothrix Wislizeni A-Iray.

1133 Jolaunm

1134 Lencio

1135 Eyminatomia multiflora

1130 Equisetimu

1139 Eryngium

1135 Verbura

1139 Eupatorium Srygui a Sray.

1140
OCT i2

San Pedro River, Hex. bonnd. Line.
I/

OCT 12

OCT 12

OCT 12

OCT 12

OCT 12

DCT 12

Oet. 15

Oct. 15

Or.15 Hear mommunt ho.26 (7. Nagme.)

Oct.15

1111 boleosauthus. 


\section{8}

1113 Potanizia

1111 bardmus

1145 Eriogromm

1116

1148 Enagrosti:

1115

1119 Solidago

1150 Prim

1151 Querens

1152

1153
Oot 15

$00 \mathrm{TT}$

Det is

OCT 15

OCT 15

OCT 15

OCT

OCT 16

Act.21

Oct. 26

nor. 4 .

1078

hov 3
Near Monumut no.26. (F. Waymer)

"

"

"

"

Sau Pearo Ruirr, Sonora, Huex.

"

"Kux. brond his .

Huachuen nus, Ariz-

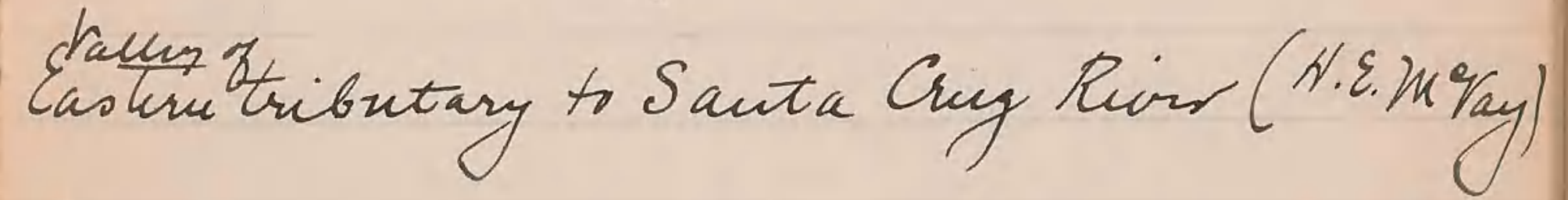
Sauta Cuz Rion at La Noria. 
1153 Castalia

1155

1156

1158

$115 \mathrm{~s}$

1159 Lepachys

1160

1161

1162 Cuchrus

1163 Argeuove

1164 Eniogrumun

1165
Sauta Cnuz Riorr at La Noria.

1118

$\operatorname{lin} 8$

Init \&

mot 8

Hon $y$

$\because$ in

ivit 8

$\therefore$ ․․ 3

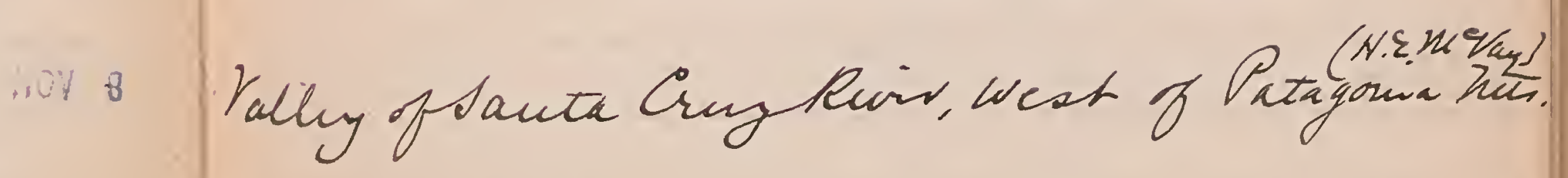

Nor.10. Forshius of Putagrmia nto. (nevay)

Nor.10

$/$ 
1166

$116 \%$

1165

1169

1180

$11 \% 1$

$11 \% 2$

1183

1184

1185

1186

$11 \%$ wis 12 Sauta Cruz River at La Noria

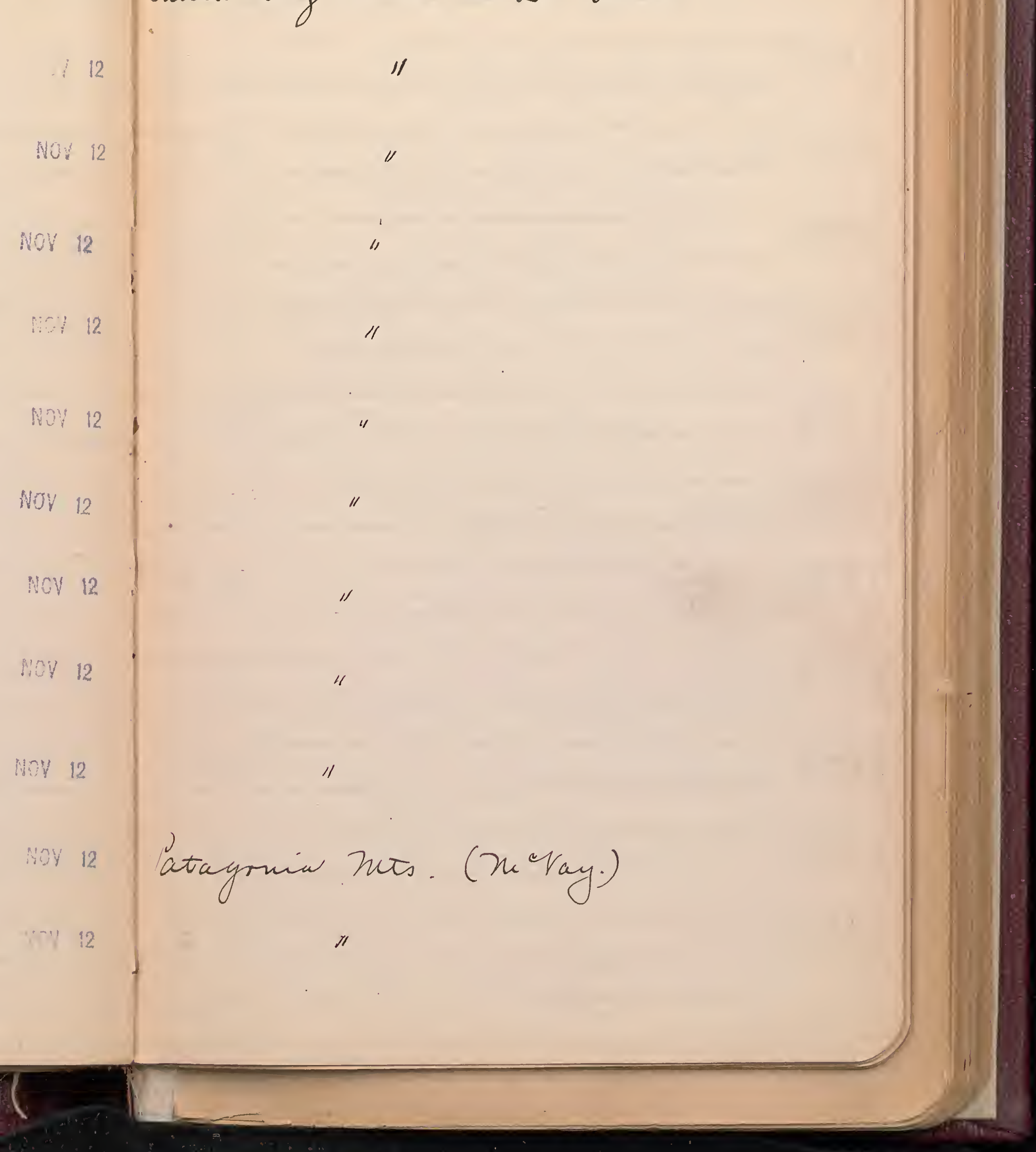


$11 \% 5$

$11: 9$ Juglaus

1180 Querens -

1181

1182

1183

1184 salix

1185

1186 Juglans

$118 \%$ Senecio

$11.8 \mathrm{~s}$

1189
$10 \times 12$

Valley of Saut a Cny River (Xucray.)

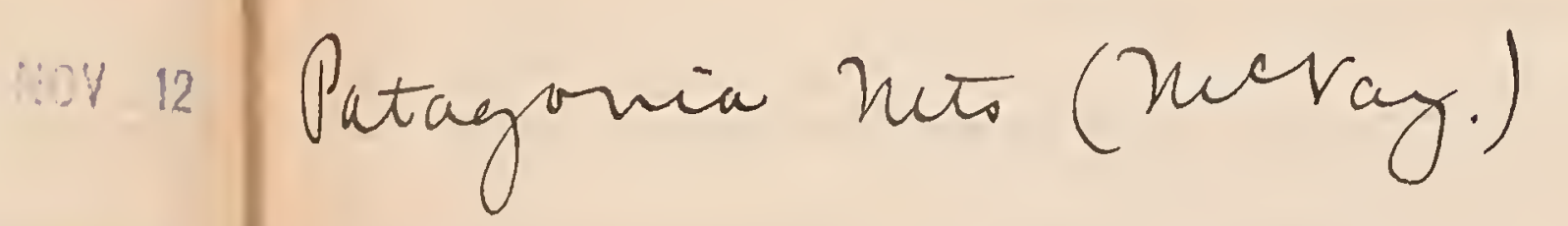

Noy 12

Nor 12

HoV 12

Nov 12

Nov 45

Sacita Guy tally (nerray)

NOV 15

Noy 15

NOY 15

Nov 15

Hov 15

woy is

- Sauta Cruz River as La Naria.

boy 18

" 
1190

1191

1192

1193 Cassia pumilio a. Fray

1194

1195

1196 Casria Raemeniaua Schulv

$119 \%$

$119 \mathrm{~s}$

1199

1200

1201
Nov 18 Sauta Cruz Rivr as La Koria.

Wov 18

Nor 18

Noy 18

noy 18

NOV 18

Nov 18

Nov 18

Nov 18

NoV 18

nor 18

NoV 18 
1202

1203

1204

1205

1206

$120 \%$

$120 \mathrm{~s}$

1209

1210

1211

1212

1213.
NoV 18

Nov 18

Nov 26

Nov. 26.

nor. 26

Yor.26

Nor. 26

DEC a Fort Clark, Minny Co, Texae.

LEC

DEC 3

DEC 3

DEC 3

Sauta Cruzhiors at La Noria.

11

'

"

/

")

" 


\section{4}

\section{5}

1216

\section{1: Cuchrus}

1

1215 Solaunum

1

1213

1

1220

11221

1

1222 Sida

11223 Castalia

1 .

$12 \%$

$1 \stackrel{4}{4}$

$1 \% 25$
Fort blark, Mininy Co, Texaer.

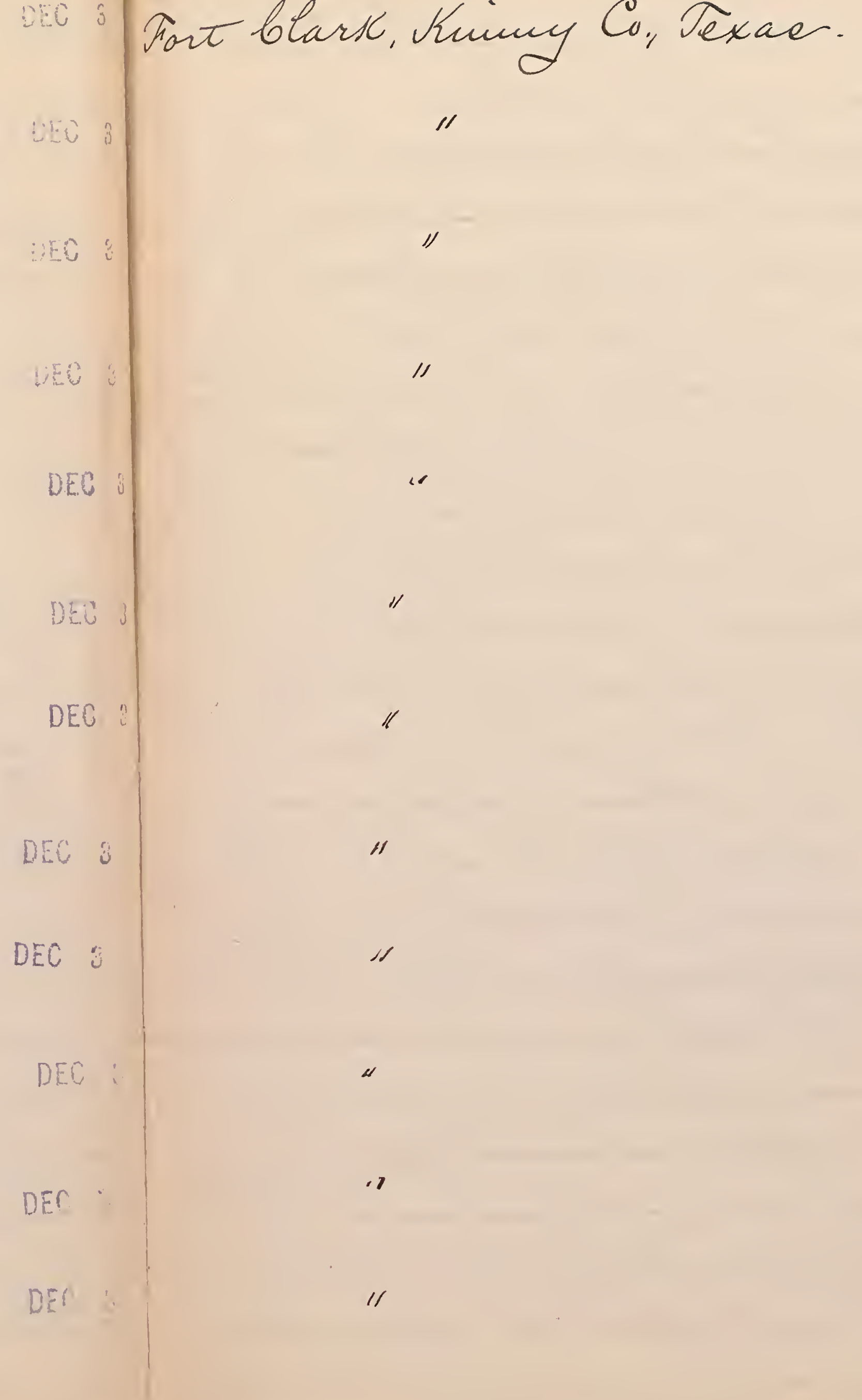




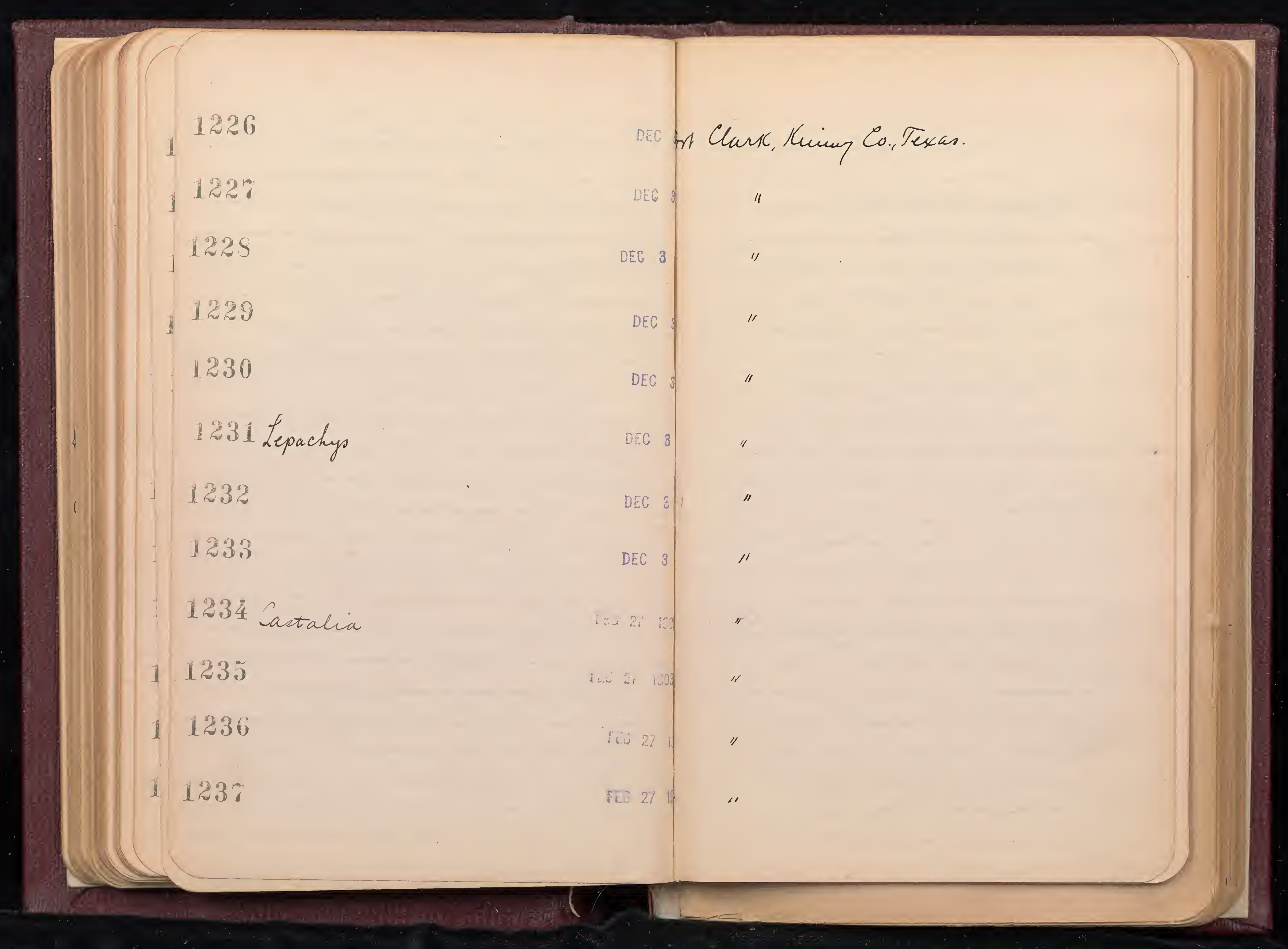


1235

1239 Anemone decafetala ard.

1210

1211

1212 Berberi

1213 Annuvectionm

123 ricia

1245

1216

1218 Erigunan

1245

1219 Samolus
Ins 27 in. Tont blark, Nininy Co, Texas.

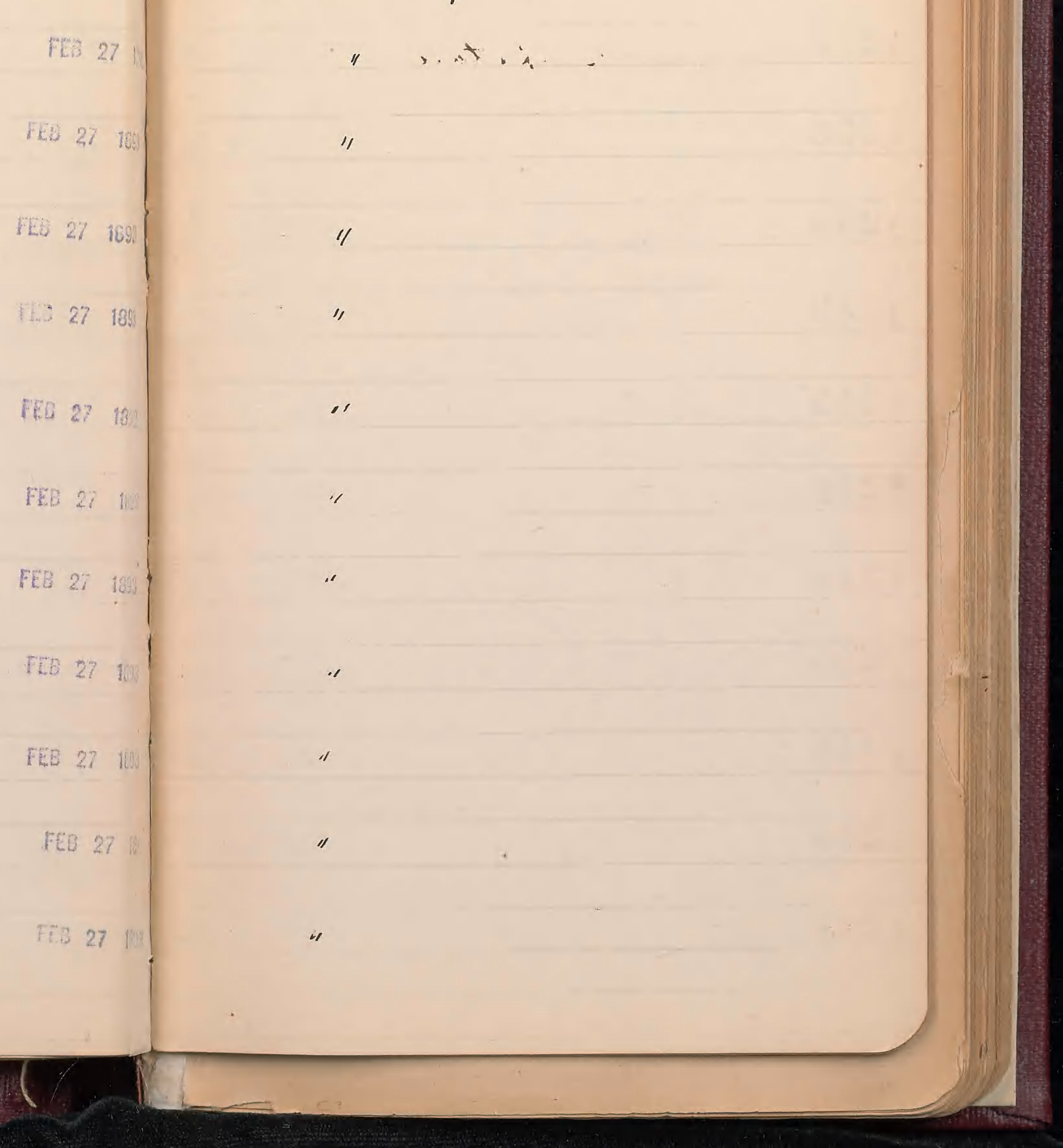


1250 Oeuothira

1251 Eenactura

1252 Vubua

1253 venoctira

1654

1255

1256

$125 \%$

1255

1259 bercis

1260 /Berberio

1261 Oxalio
FEB 27 1980 Fort Clark, Kuiny Co, Texar.

- FE] $27 \quad 1893$

Near. 3, 18\%3

$-18.5 \quad 5 \quad 1893$

$\not$

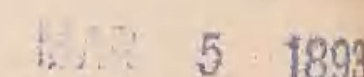

mas. 7.1893

Wมล $10 \quad 1893$

Wha $10 \quad 1893$

44. $10 \quad 1893$

Whas $10 \quad 1993$

MAR $10 \quad 1893$

Mrab $10 \quad 1893$ 
1262

1263

1264

1265

1266

$126 \%$

1265

1269

$12 \% 0$

12:1 Scutellaria

1272 Annusshimmo

1283
With 101893

Font lelark, Muning leo, Texas.

MAh $10 \quad 180$

MARs $10 \quad 189$

MAR $10 \quad 180$

MAP $10 \quad 1893$

MAR $10 \quad 189$

WAA $10 \quad 1898$

MAS 101893

WA $10 \quad 189$

WAR $10 \quad 189$

What 30700

Whn $10 \quad 1893$
/I

11

"I

,

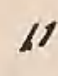

II

11

"

"

"I

" 
$12 \% 4$ Yerbena

1285

$12 \% 6$ Jencio

$12 \%$

$12 \% 5$

$12 \% 9$ Anemone decapetala and. 101898 1280 Drabu flatycarfa Int hry. Mas. 101893 1281

1282 Verbeua 1283

1284

1285
MAR 101093 . Fort lelark, Muiny Co, Texas.

\section{MAR $10 \quad 180$}

What $10 \quad 1893$

MAIR $10 \quad 189$

MAR $10 \quad 1893$

MAR $10 \quad 1893$

WAA? $10 \quad 1893$

20101003

$M P: 10 \quad 1809$

$R=10 \quad 1933$

Whas $10 \quad 18$ X
"

"

.

"I

" 
1286 morns

128\% Prosopis juliffora

1285

1283

1290 Uncea

1291 Castillua

1292 Leranimm

1293 Plautago

1294Parki

1295

1296

$129 \%$ Delphiminn
Mareh 11,1893 Fort blank, Kümy Co., Texare.

harch $\mathbb{R} / 893$

Mauch 12,184/3

hauch 13,1843

$\therefore \quad \therefore \quad 1393$

16: 131893

Mst is 1993

Wh? is 1893

Hen $15 \quad 1099$

math the 180

(1).... 13:3 4

Whit is 1893

(P.
/I

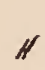

"

‘

" 
1295

1299 Clacia

1300

1301 Lupinus

1302 Castalia

1303 Kynuphaw

1304

1305 Rabua

1306 Phus

1307

$130 \mathrm{~s}$

1309
151993 Trot blark, Kuiming Co., Texas.

MAB $\quad 15 \quad 1893$

WAR $15 \quad 1893$

MAR $15 \quad 1893$

in 1890

MAR $15 \quad 1893$

MAR $\mathbf{6} 1893$

What if 1893

When 191893

$562 \quad 19 \quad 1893$

MSA $19 \quad 1898$

MA8 19189

MAP $19 \quad 180$

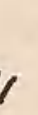

(n)

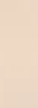

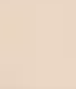


1310

1311

1312

1313

1314

1315

1316

$131 \%$

1315

1319

1320

1321
H: 19 180i Fort blurk. Kuiny Co, Texas.

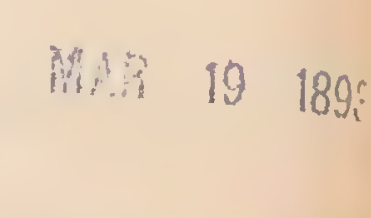

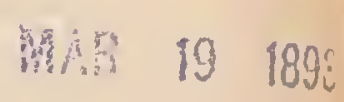

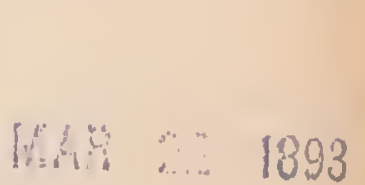

thit. .... 1893

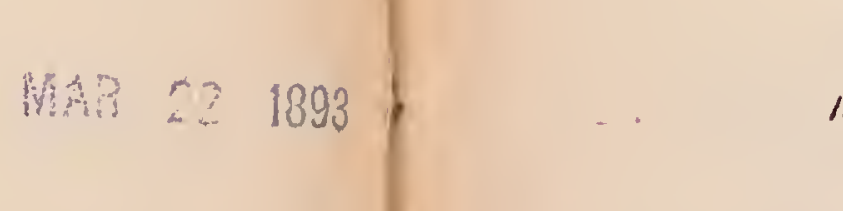

MAR $22 \quad 100$

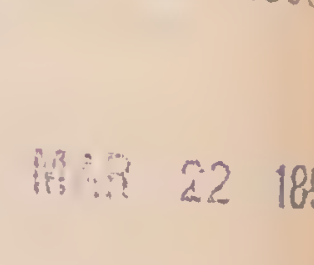

ins 22

MAS $22 \quad 1093$

18,3

Wha $22 \quad 1893$

48221893

(1) $22 \quad 1893$
11

1

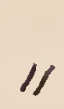




\section{2}

1323

1324

1325

1326 Querens

1327 Phacelia

1325

1329 Plantugo

1330 Metir

1331

1332

1333 barlowrightin
221893 Trot lelark, Muniny Co, Texas.

MAM $22 \quad 1893$

MAR 221893

MAP $22 \quad 1893$

(4) $22 \quad 1898$

MAP 221893

MA? 221893

MAI $22 \quad 1898$

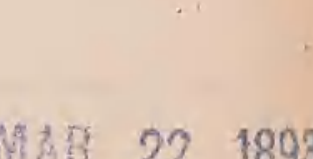

MAn 221893

MAก $22 \quad 1893$

MAR 22. 1895

MAस 221898

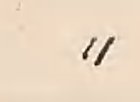

II

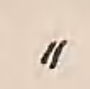

k

II 
1334 Sentellaria

1335 Verbux

1336

1337 Prosopin juliflora

1335

1339

1340

1311

1312

1343

1344 Clenatis

1345

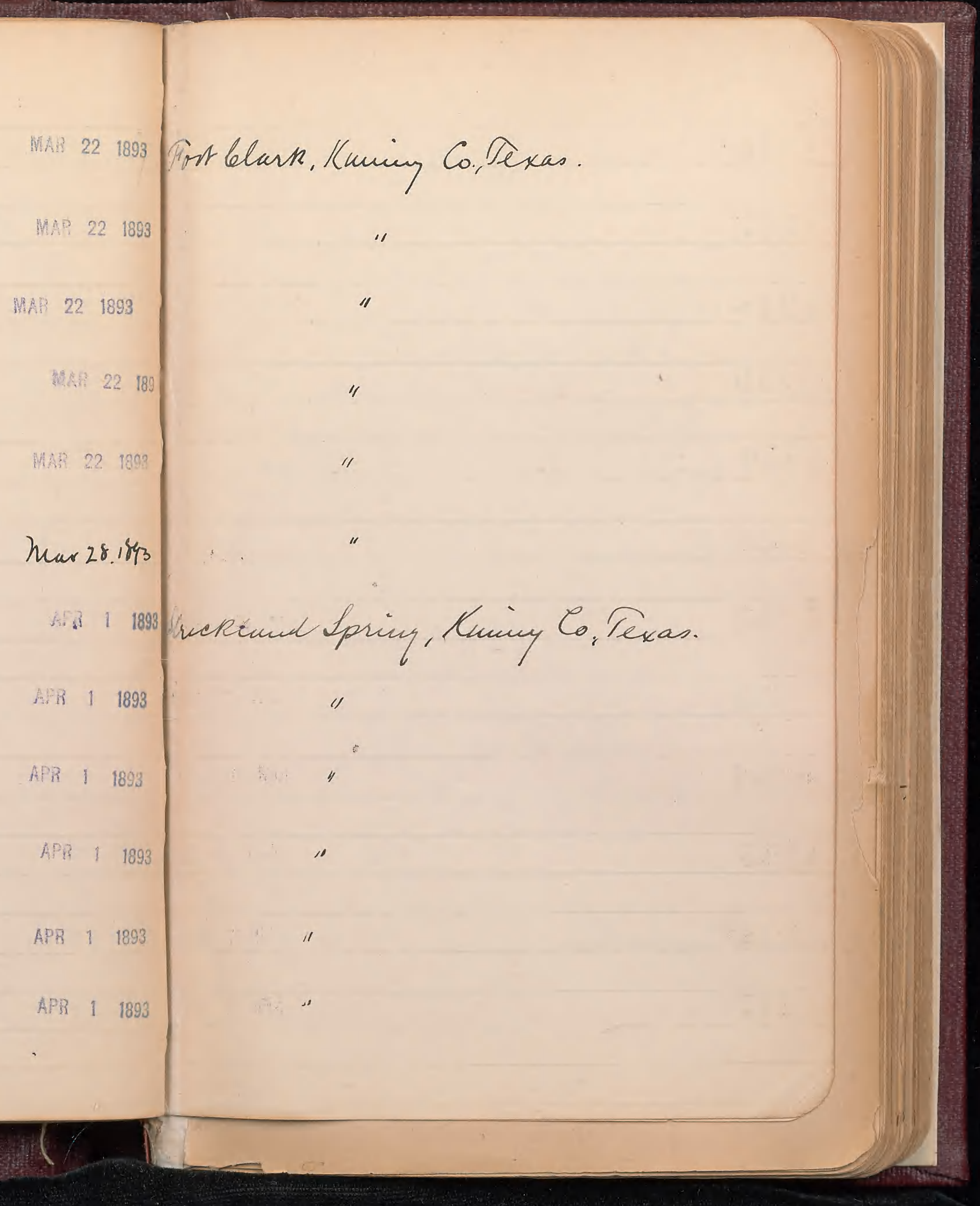




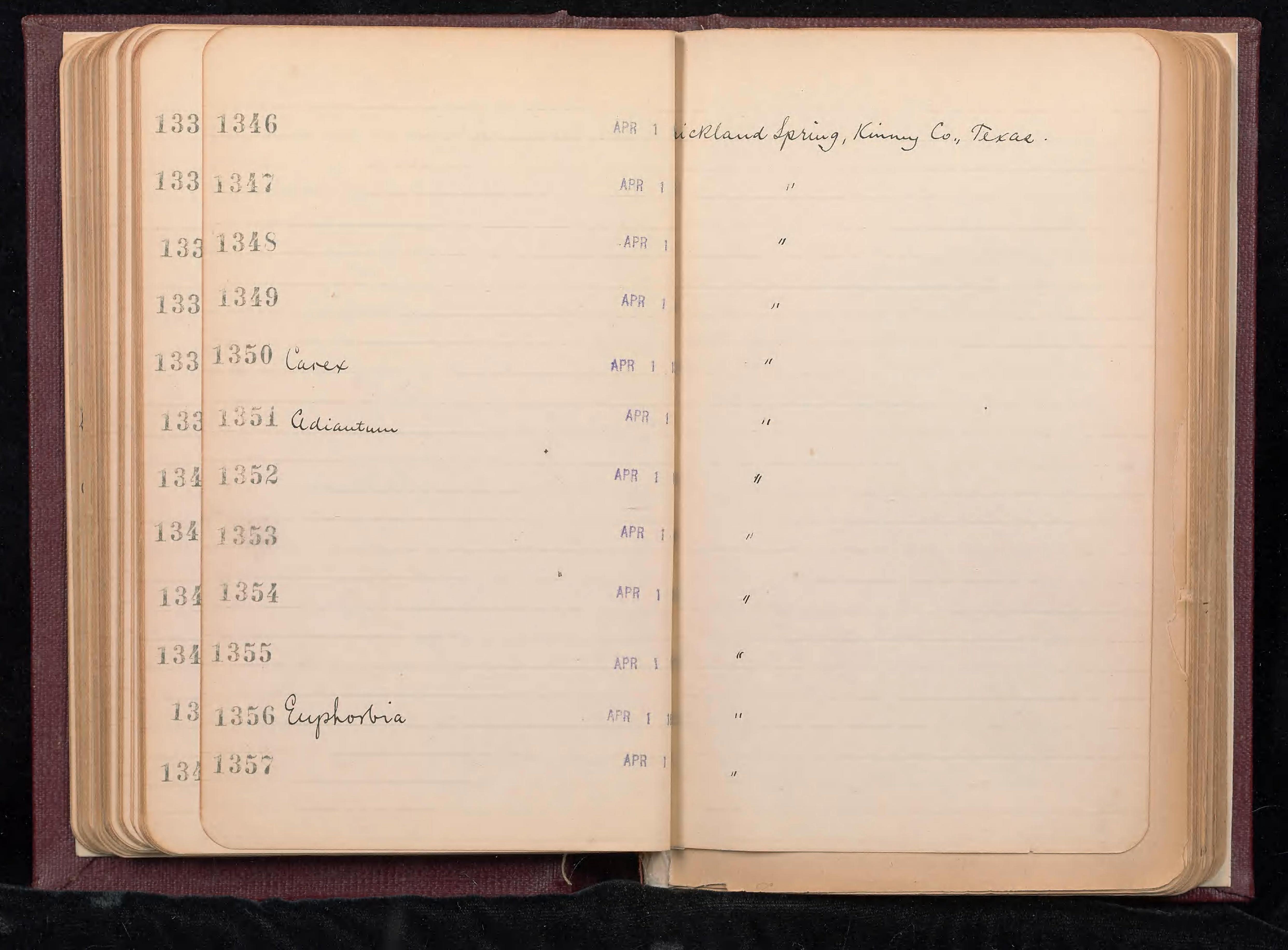




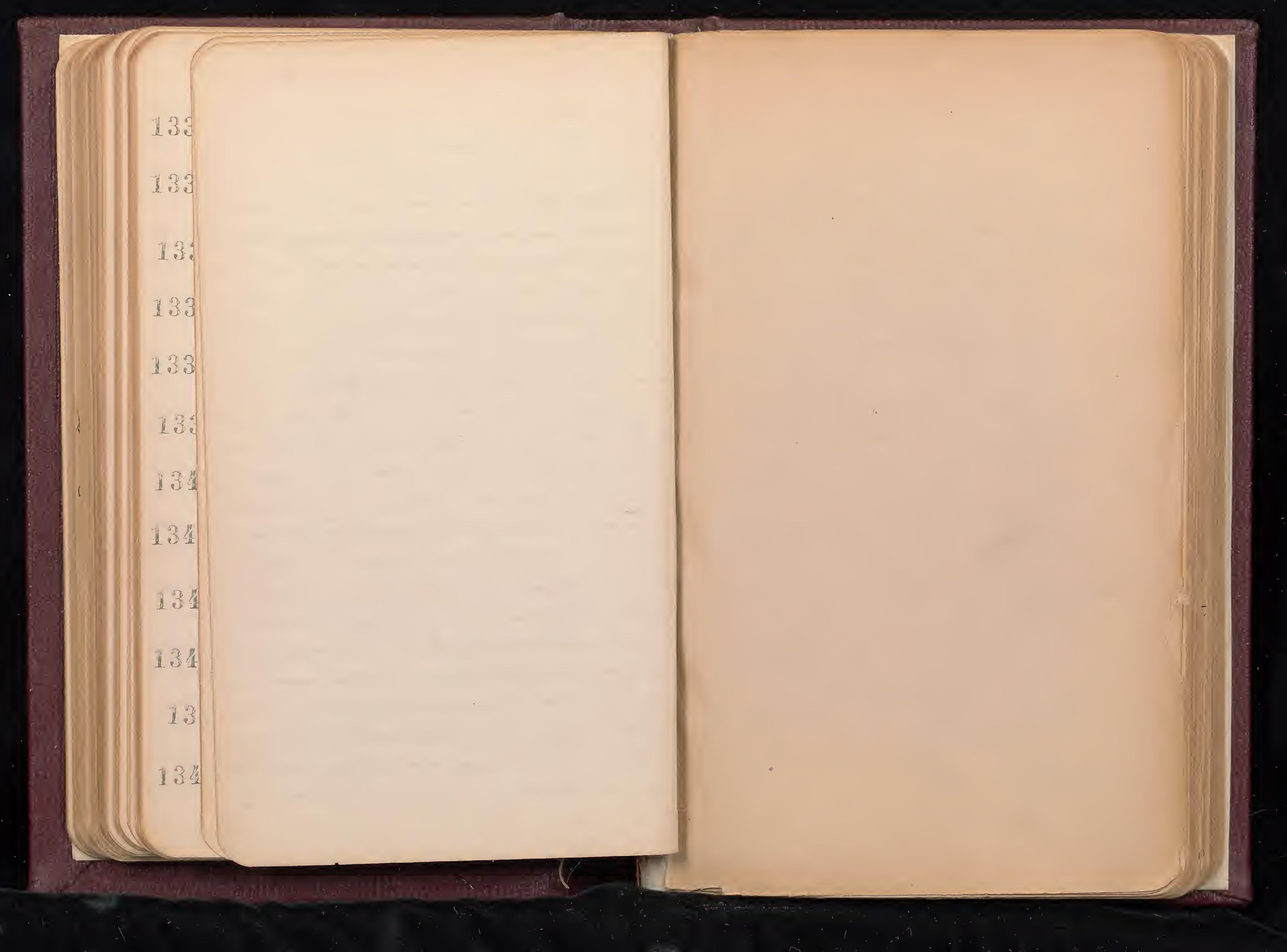




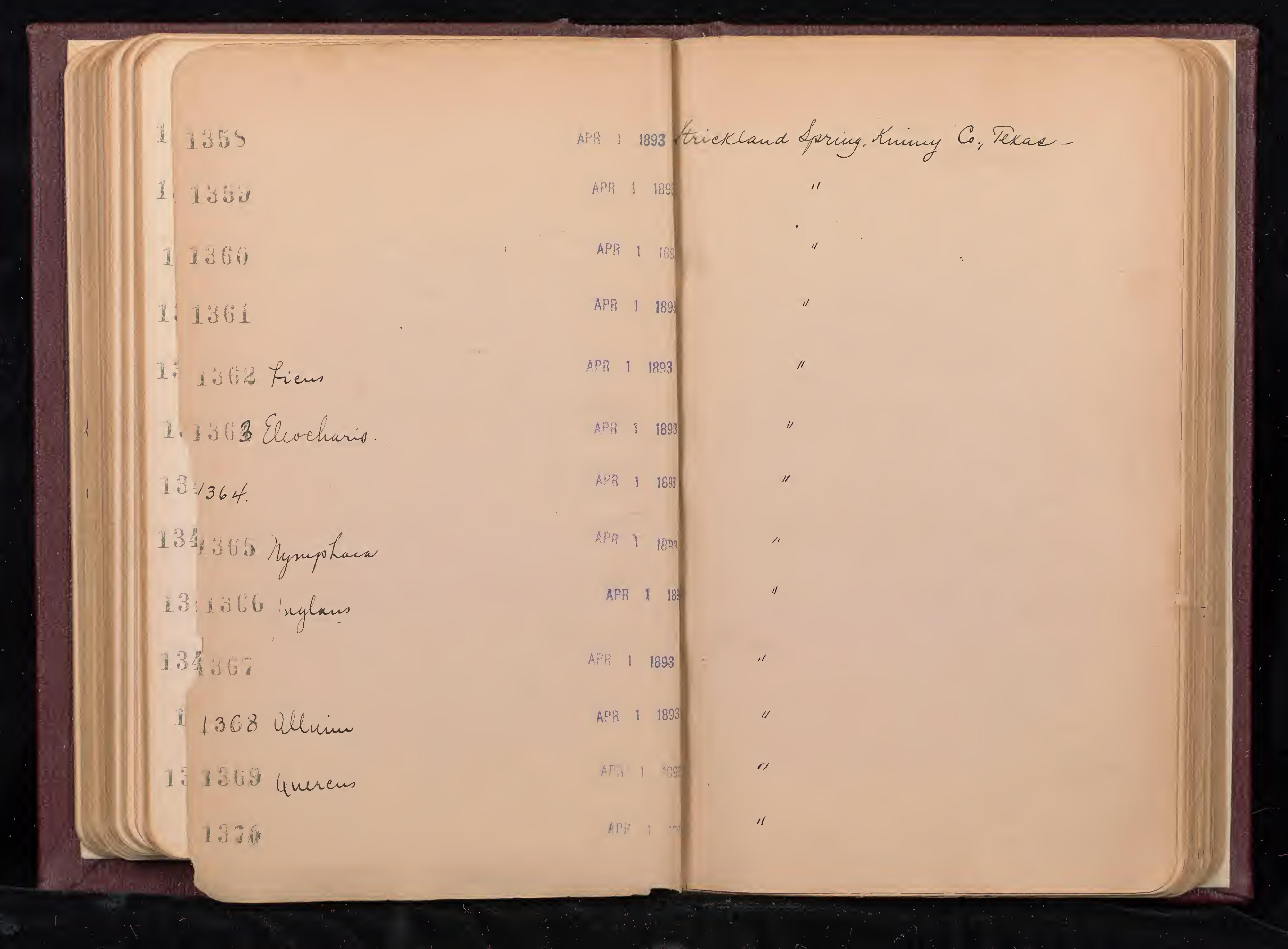


$13 \% 1$

1) कै

\section{3}

$113 \% 1$ Oenothon

1.1060

1386

$1: 13 \%$

131335 hietia Gadaract 2.

131392 Licm

131580 velphinim

1381 Parosula

1: 1 :8?

1883
APR I 1893 Strickeand Lpring, Nhing Co, Txxas.
APR I 1898

APR I 1893

AFR $1 \quad 1893$

APR $\quad 1 \quad 1893$

APR $\quad 1 \quad 189$

APR $1 \quad 1893$

APQ 11893

$A P R$ i to

$A P R+T Q D Q$

$A P R \quad 1 \quad 139$

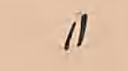

'

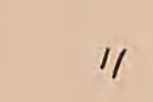

16

"

11

i

11

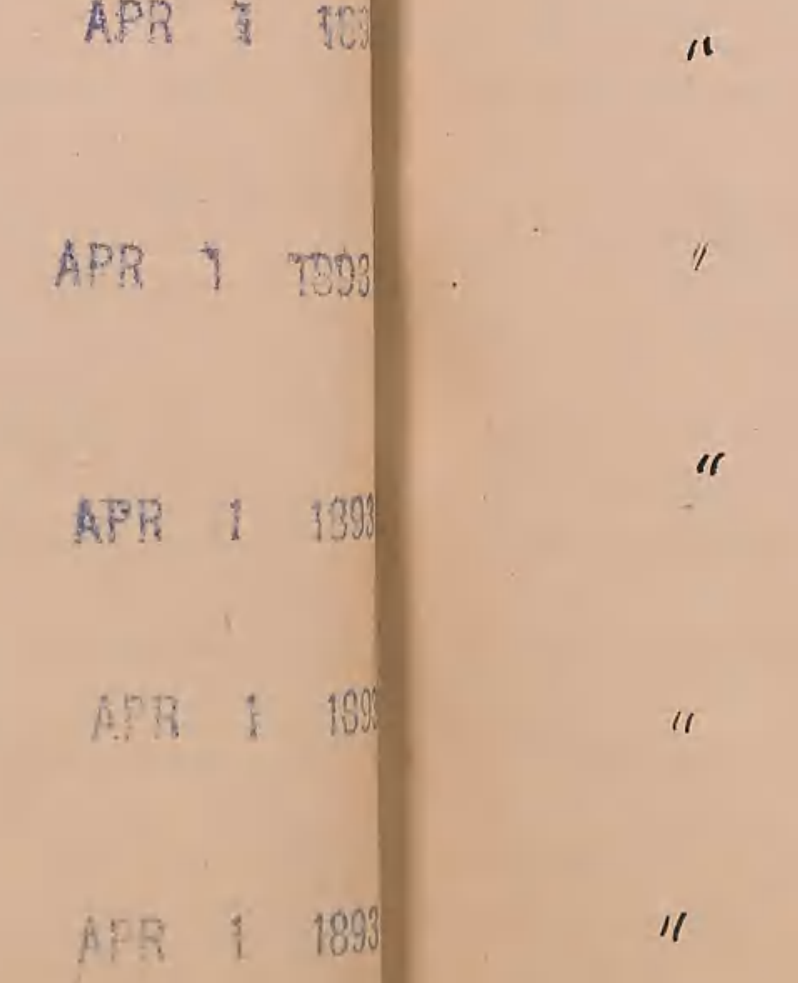


1384

1395 Prosopis julifcora.

1386 Cassia pumilio G. Sray.

$135 \%$

1285

1399

131390 Plantago

131391 Sencio

1392

131393

1394 Tirbua.

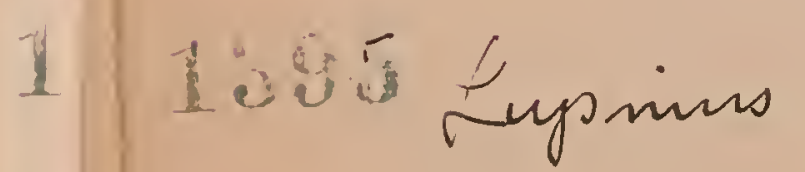

1396 Thus trilobata
ADR I S993 Stricklaund Spring, Kinury Co, Texas

APp $\quad \uparrow \quad 1393$

Asis Fortblark, Kunny Co., Sexas.

$43 \quad 3 \quad 1893$

APR S 189

APR $3 \quad 1998$

APP

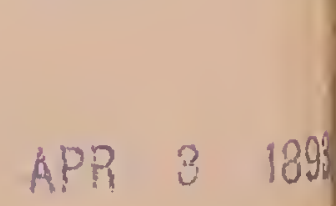

APF 31893

(4) 318

APR $: 1893$

APR - "2033 


\section{Maloa}

1411 Anoda

\section{$141 \%$}

1413 Inclia Azudaruch 2.

1414 Lepidinum

1415 Cuercus

1416

$141 \%$

11415 Physalis

\section{9}

$142 ?$

1421

1422

\section{ARR 31893 Fort Clark, Knimy Co, Texas.}

Af? $3 \quad 1893$

APR 31893

APR $3 \quad 1899$

APR $3 \quad 1898$

APR $8 \quad 1893$

APR $22 \quad 1893$

APR $22 \quad 1893$

APR 221893

APR 2? 1993

APR $22 \quad 1893$

APR 221893

APR $22 \quad 180$ 


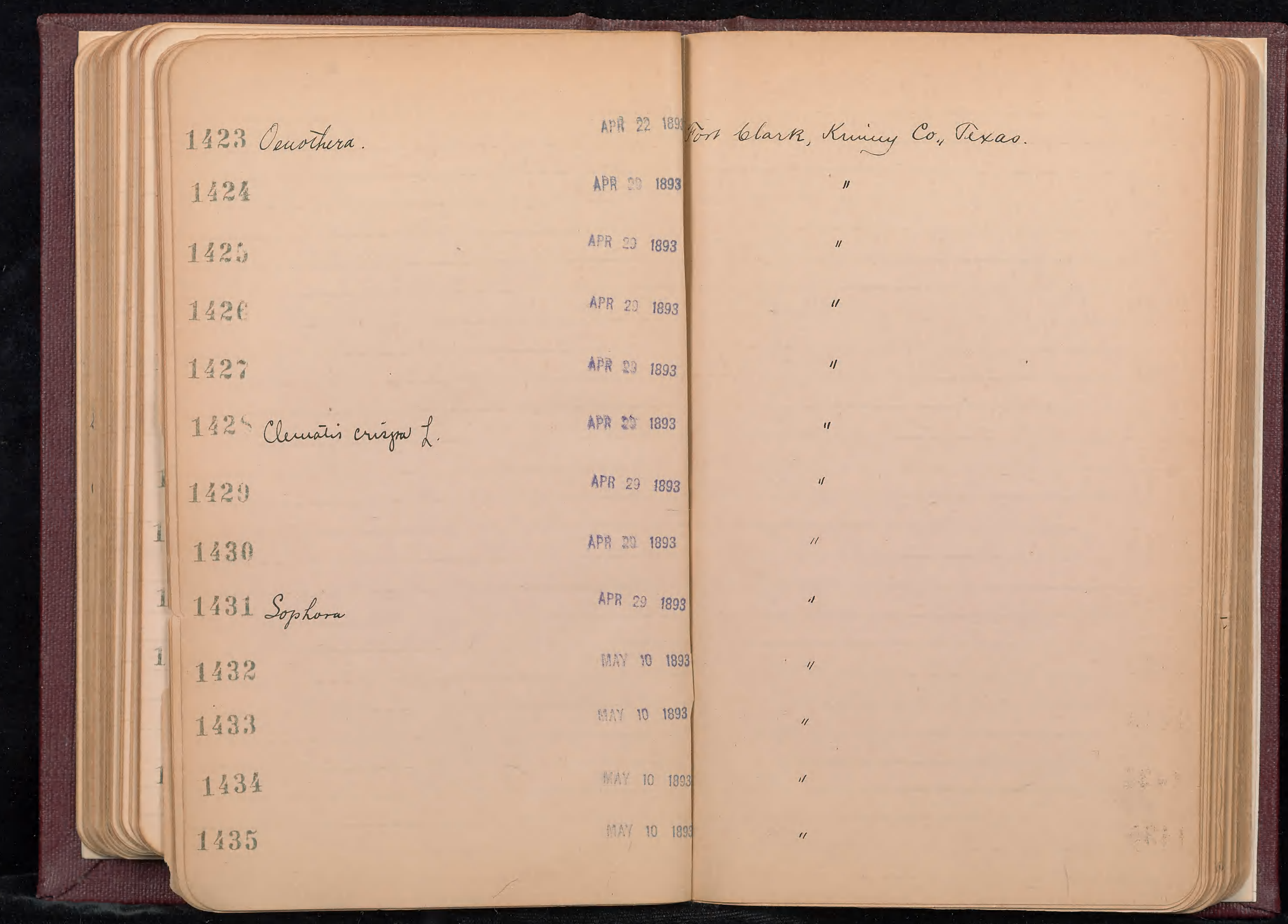


1449

1450

14 Parosula

$145 \%$

1453 Carsia Roemerianal Schurs

1421 barlowrightia

136

1456 Vubua

\section{8}

1459 acacia

1459

1160 Sisyriuchum

1461

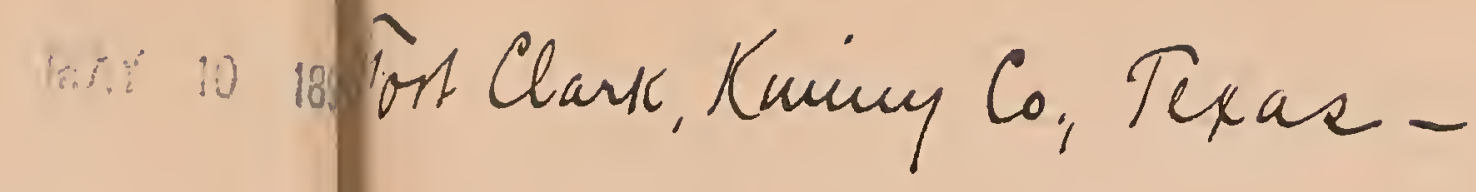

MAY 1018

WA: $10 \quad 1893$

Wy 10 189 11

19:A 10 189

HA $10 \quad 1893$

Wy: 10 1893 "

Mat $10 \quad 1893$

MEY $10 \quad 1893$

MEX $10 \quad 18$

: 1909

Ne 101893 
$14 \dot{6} 2$

1463

1461 Snilax

1465 Prosopis juliftora

1466 Juglans

1467 Inpachys

1465

1469

1420

1481

$142 \%$ Reliotrapinn

1483 Bacchanis

148
Mar 10 In Clark, Kunung Co, Texas.

MAY 10

MaY

MAY 10

MAY 10

MAY 10

MAY 10

MAY 10

MAY in "

- Paro, Texas. 
1485

146 Cansalpinia

$11 \%$

$11 \% 5$

$14 \%$

1480

1481

148?

$1 \div 83$

1354

1495

1.486

1488
Paso, Texas.

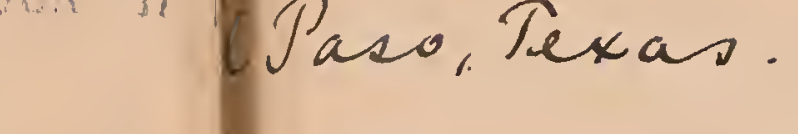

$11 . ; 17$

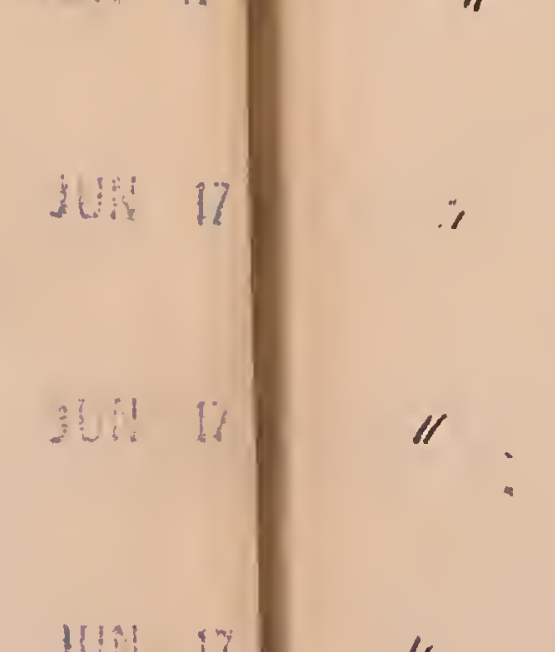

Wuin

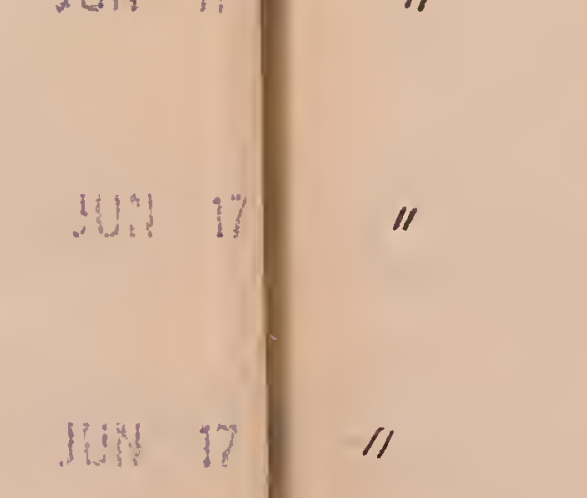

Jin 17

$3 .+3 i$

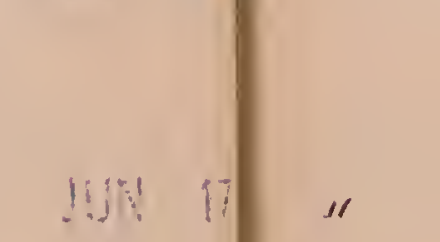

IIf 10

19017

WW "

was

(4) 
1485 Prospin puberceno

1489 Prosopis julíffora

1490

1491

1492

1493

\section{Sabbatia}

\section{5}

1496

$149 \%$

1495

1499 Covillea tridentata

1500
Jux 17 El Paso, Texas.

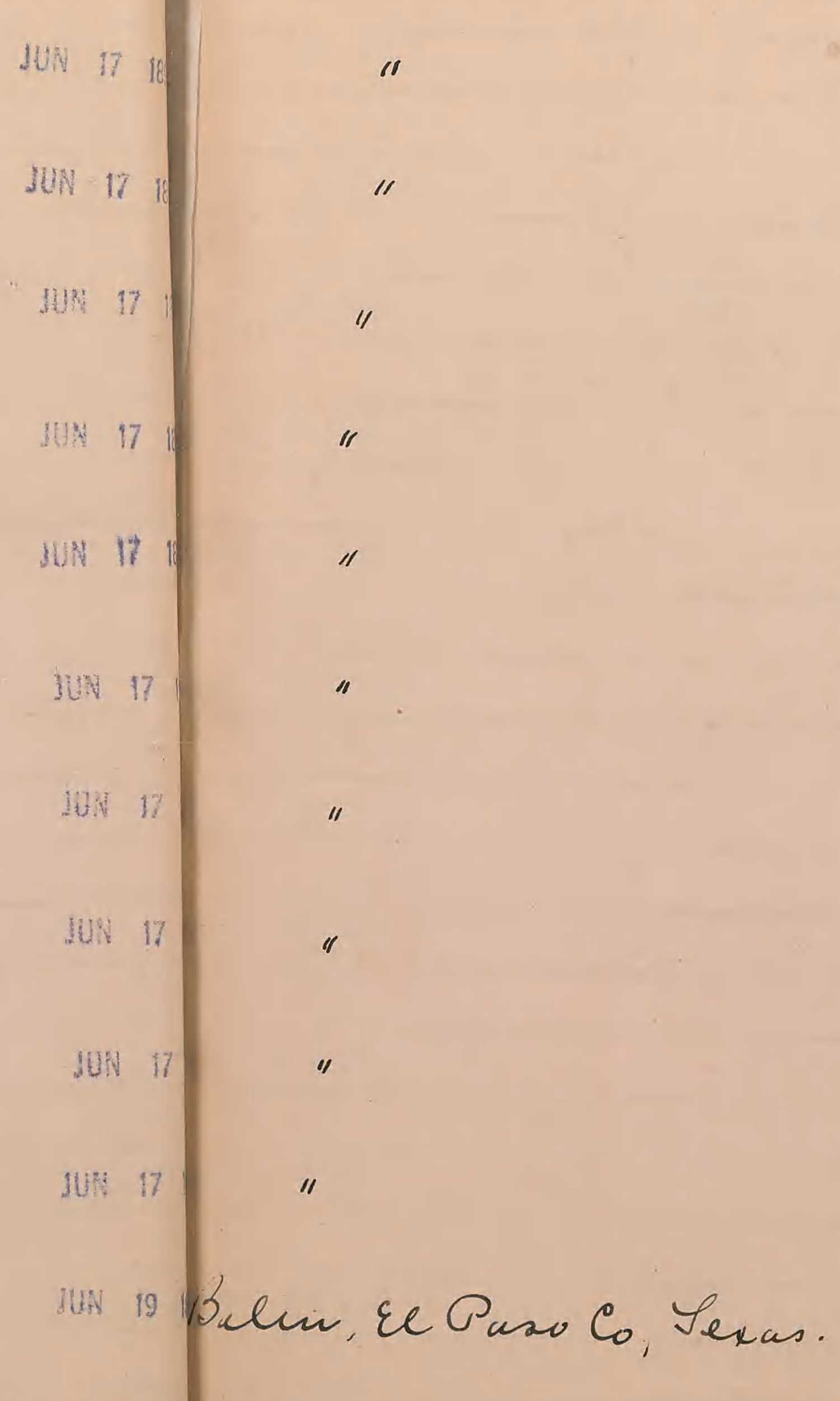


1501 bavalpinia

1502

1010.3 Anvaranctus

1501 Anearanthus

1283

1106 Prosopis pubescens

1507 Prosopir juliflora

1505 Lippia

1009 Bacekani

1 1.10 Solanum

1. 1. Salix

1 12. Papulus.

13li Anclepias
In: 1993 Belu, El Pars Co Texas.

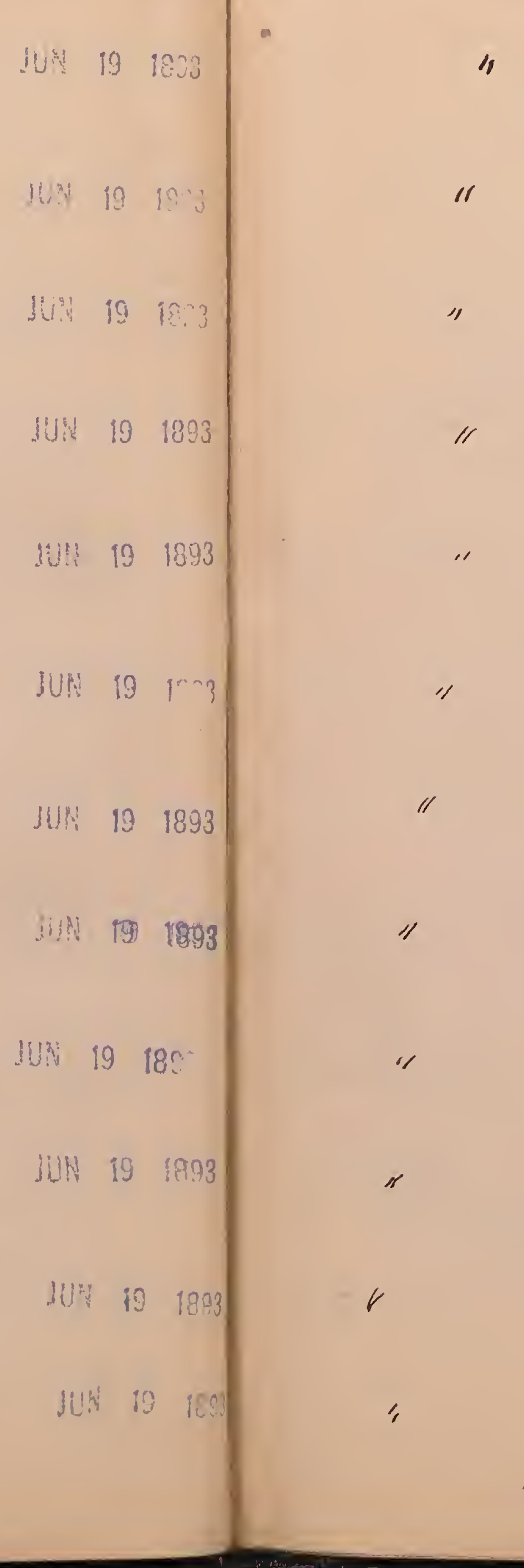


1511

1515 bardune

1511 Sepidium aly ssoides A. Any Jun 191893

1.51: Lepactys

1515 Solacums elacagnifolinis Car.

1519 Delphuivin

1520

1521. Prosopis puberens

$152 \%$

1523

1524

1525 Zinue

$52 \%$ Jenchura

JUN $19 \quad 1893$

JUN $19 \quad 1893$

JUP $19 \quad 1893$
JUN $19 \quad 1693$

J414 $\quad 19 \quad 1893$
Beleu, El Pass Co Texar.

Inue 25,1843

$\operatorname{gmen} 251893$

Znum 30.1893

nly'.1893. Sierra Blauca, Dexas.

July 151893

Jul 15,843 Strans' Station, New mexics

Jiny 26.1893 Huachuea Nits, Aryona. 
152:

1529

$$
\text { Lily } 26,18 \% 3
$$

Fort Huactuca, Aryzina

JUL 27 189

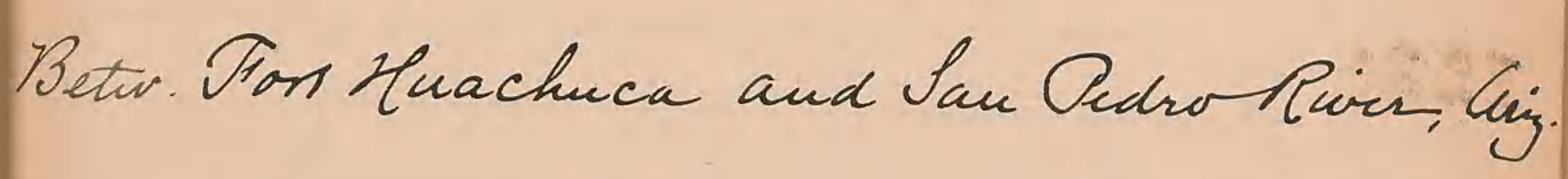

1529 Actaca viridi flora Bnem JUL 271893

1530

1531 Potuticla

1532

1533 Yucea

1534 Solaumm

1535 Rhus

1536

1537

1539

1539 Castillyo
JUL $27 \quad 189$

JUL $27 \quad 1890$

JUL $27 \quad 18$

JUL 27189

JUL $27 \quad 189$

JUL $27 \quad 1893$

JuL. $27 \quad 189$

JUL $27-190$

JUL 27 i8

JUL. 27 
1540

1541 Draba petrophila Enime.

1522 Luranim

1583

1514 Ayume

$15 \$ 5$ Populuo

1546 Frasera

1548 Pnenmaria

1549 Querens

1549

1550 Asclepias

1551

1552
WUL $27 \quad 1893$

Betwr. Fort Herachuea and Sau Osdro Rim, Ariz

JUL $27 \quad 1893$

JUL $27 \quad 189$

JUL $27 \quad 189$

JuL $27 \quad 189$

JUL $27 \quad 1893$

HUL $27 \quad 1893$

JUL $27 \quad 1893$

JUL. $27 \quad 1893$

Trachuca Into Ariz

JUL 30189

San Oedro Rivin, Mux. bonner liur.

JUL $30 \quad 189$

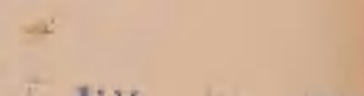

(1) 104, $3: 18$

Huachuca Nut, Arizima

\section{2}


1553 Leranim

\section{4}

1555 Peutstumin

1556

1557 Villa Canadunantis

1559 Lymphoriearpos.

1559 Sambuans

1560 Prudotruga taxifolia

1561 íclepias

1562

1563

1564

1565
[ JUL 31 1893

JUL $31 \quad 1893$

JUL $31 \quad 1893$

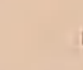

JUL $31 \quad 1893$

JUL. $31 \quad 1893$

JUL $31 \quad 1893$

JUL $31 \quad 1893$

JUL 319893

AUG I 1893

AUG $\quad 1 \quad 1898$

AUG $1 \quad 1893$

AUG 1 189:

AUG 11892

Huachuca huts, Arigona.

1"

I

$$
\text { Lighest seak }
$$

second Lighut peak.

milleris Caî̀n. Jluachuca not.

Huachuea huts - Leeond highst pearc.

"

second Lighest peak.

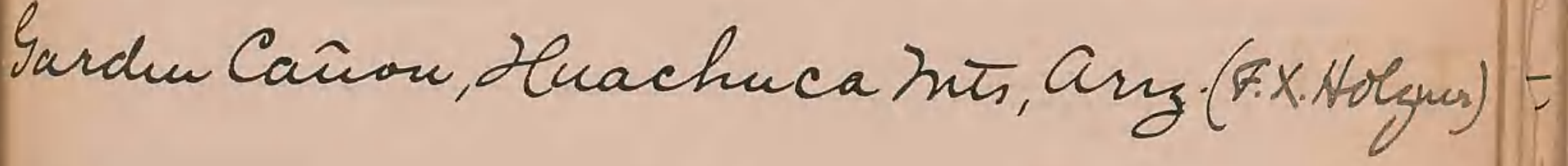
Tauner: Cañon

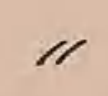




\section{6}

$156 \%$

1569

1569

$15 \%$ Asclupias

1581

1592 Palygala

\section{3}

1581

1585

1276 acacia

$159 \%$ Heuchera

1598 Solanum
AUG I 189: Samuer's bauon, H(uachurca hita- Arig. (F. X Holgun)

AUG $\perp 189$

AUG $2 \quad 189$

AUG 21893

AUG $2 \quad 1898$

AUG $2 \quad 189$

AUG 21893

AUG $2 \quad 1893$

AUG $2 \quad 18$

AUG $3 \quad 18$

AUG $3 \quad 1893$

ALG 3

AUG $3 \quad 1893$
II

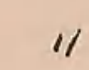

"I

11

" 


\section{$15 \% 9$}

1580 Bouvardia triphylla

1581 . ris

1582 Prumus

1583 Rhus

1584 Quercus hypolenca

1585

$1584 i$

$158 \%$ Ptelea

1584 Sapmuphoricaupes

$158 \%$ Berbers Wilcoxï Kenmy

1590 Prims Arizonica

1591. Prim
AUG 51893

Alto 31893

AUC 31893

AUG $3 \quad 1893$

Ang 31893

Ave 31893

AUG $3 \quad 1893$

AUG $3 \quad 1893$

AUG $3 \quad 1893$

AUG $3 \quad 1893$

AUG 31893

H.x is 1893

AUG $3 \quad 1893$

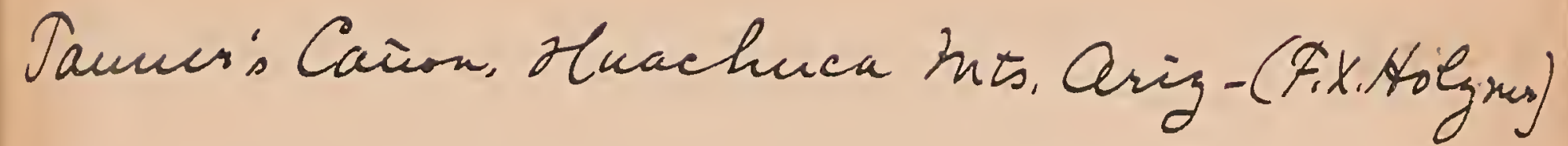
!!

Sau gosé Into, Sonora, Mux -

15iling 
1592

1593

1591 Pppulus tremuloidus DC $159 \%$

1596

1598

1589

1599 Solanmm

1600

1601 Oxalis

1602 Heuchira

1603 Seranim

$160 \%$
AUG 31893

AUG 31893

AUG $3 \quad 1893$

AUG $3 \quad 1093$

AUG 31893

AUG $3 \quad 1893$

AUG $3 \quad 1893$

Aur 31893

AUG 31893

Aiv ? 1893

AlG $3 \quad 1893$

MG 21893

Allo 31893
San Jose' Mts, Sonora, Muxico.

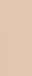

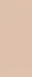

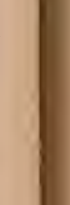

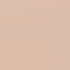

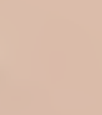

at smmit.

at sumuit 
1605 Thalictrum

1606 Oenothera

$160 \%$ Brwardia

1608 Lychrum

1609 Comundina diauthefolia

1610

1611

1612 Psendoajmpterur

1613 Psendocynepterm

1614

1615 Thammes

1616 Lraba

$161 \mathrm{~g}$
AUG $3 \quad 1893$

AUG $3 \quad 1893$

AUG $3 \quad 1893$

AUG $\quad 3 \quad 1893$

AUG $3 \quad 1893$

AUG $3 \quad 1893$

AUG $3 \quad 1893$

Aug $3 \quad 1893$

AUG $\quad 3 \quad 1893$

AUG $3 \quad 1893$

AbE $3 \quad 1893$

AUG $3 \quad 1893$

AUG $3 \quad 1893$
Sau foré mto, Sonora, Wuxics at Sumnit.

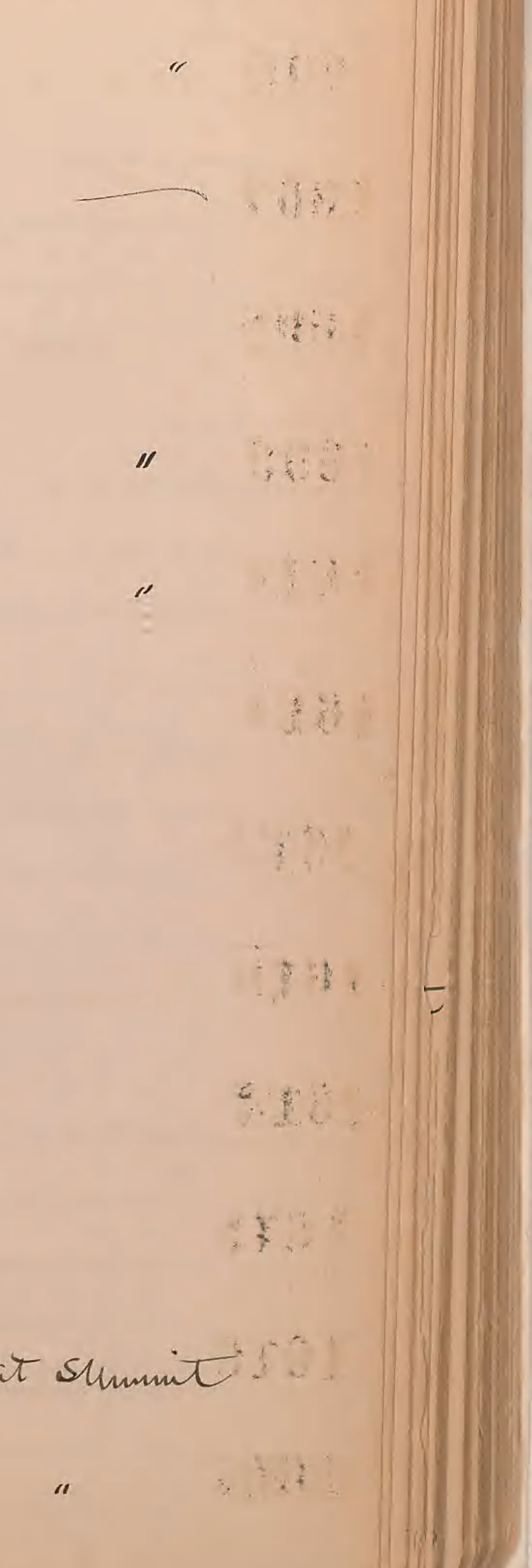


1619 Anpinus

1619

1620

1621

103 Anupirus pachypheoen

1623 Prius

1621 zuglans

$16 \%$

1626

162\% Poza

1625

1629 Querens

1630 Quercus
Alla 3 10: Sim Gose' het, Sonora Mexico.

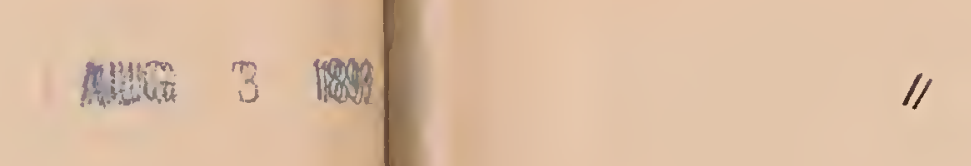

AUE is 1893

AUG 31893

at Scunnit

Auc $3 \quad 1893$

AUG $3 \quad 1893$

Aug 3 i893

at sumit

AUG $3 \quad 1893$

AUG $3 \quad 1893$

AUG $3 \quad 189$

AUt 3 189

就 
1631. Querens

1632 Quercus

1633 a. Querens

$1633 \mathrm{~b}$. Quercus

1634 Phoradudion

1635 Solamum

1636

$163^{2}$

1639

1639 Limum

1610

1681

1682

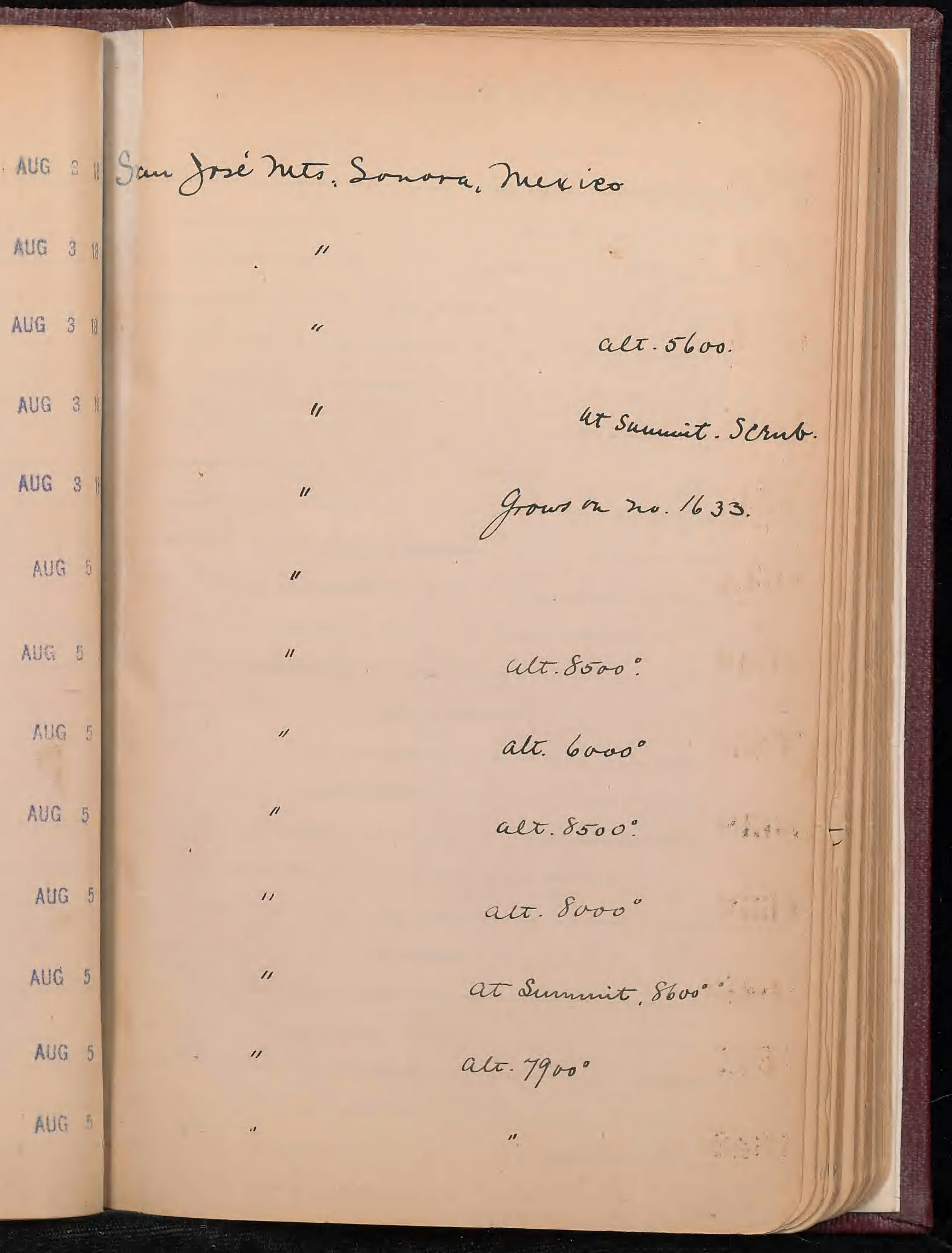


1613 Symphoricarpos

164

1615 Eraminm

1616

1619 Cyperus

1615 Robinia

1649

1650

1651. Seutstemon

1652

1653 Sambucus

1651

1655 denchira
AUG 5189

AUG $5 \quad 1893$

AUG $5 \quad 18$

AUG $5 \quad 18$

AUG $6 \quad 18$

AUG $6 \quad 18$

AUG 6 is

AHG 6

AUG

Aug 6

AUG 6

AUG 6
Surfosie hus, fonora, neex. ale. $8500^{\circ}$ :

at sumuit

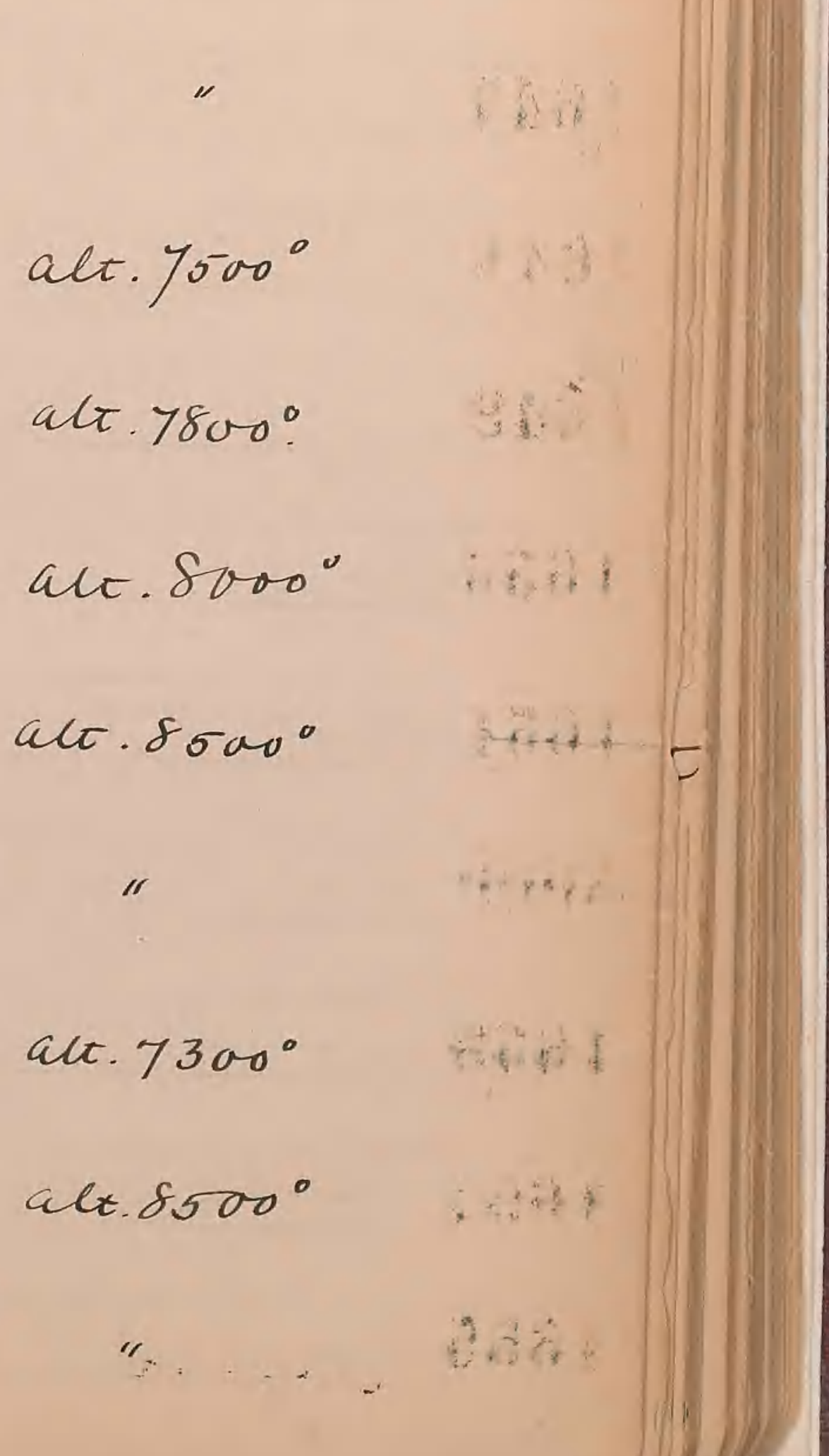


18060

$165 \%$ Lupinus

1659

1659

1860

1661

$166 ?$

$180 ?$ Attian

1 (1) 4 Pettaca

1 Gingerms

Iifif bonunctina

$4 \frac{12}{8}$

76 \& \& ingown

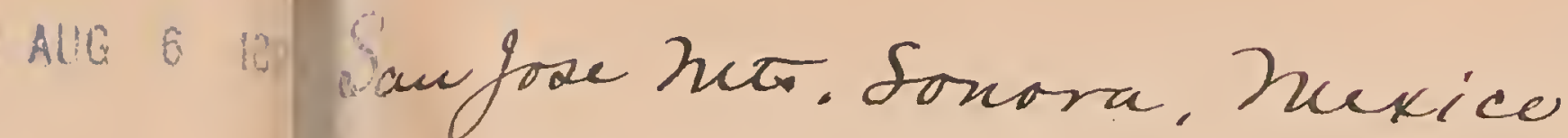

AUG 618

AUG $6 \quad 189$

AUG $6 \quad 1893$

AUG $6 \quad 189$

AHG 6 ig

Alv 6

Ais of 100

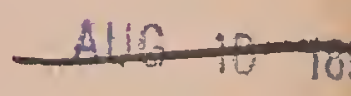

AUG $10+1000$

$-416+180$

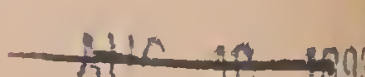

"f
Heachuca Ho, Ariz. (F.X.Aolgner) 
1663 Castillya

1664

1693 Iradescantio

1666

1669 Oxybuphis

1685

1669 Carduns

$1680 \mathrm{gnglam}$

$16 \% 1$ Paximus

16.8. Salium

1683

16\% Pins

$16 \% 5$
LU: 18: Sau Gosé Into, Sonora, Mexico. So00

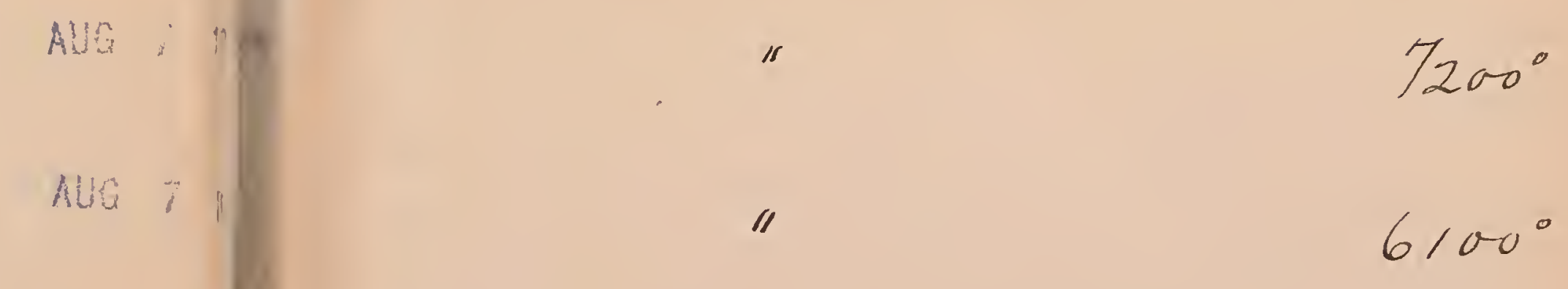

AUG : 18:

0000

Aus i 10

AUG 7 10

HUG 7 18: " 1400

AUG 7

MUG 7

AUG 7

AUG 7

AUG

Aแล 
1686

\section{$16 \%$}

1695 Epctobium

1699 Aquilegia

1680 Leramin

1681 Eriguro

1682 Potuntiera

1683

1684 Penteteruon

1685

1686 Cenchra

165 .

1685
AUG 7 10. Smin fose' mins, Sonora, mexico

AUG $7 \quad 180$

AUG ₹ 1808

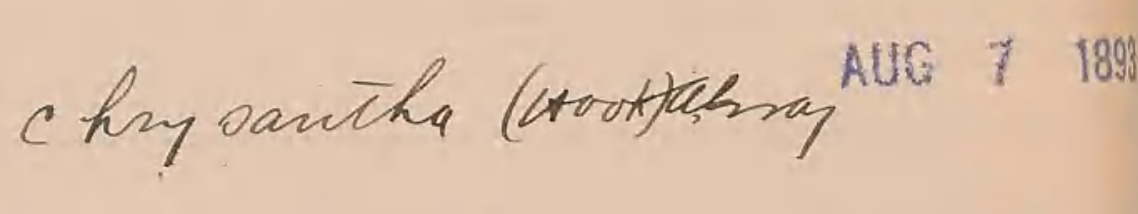

Aug $7 \quad 18$

AUG 7 180

RUG 7

AUG $7 \quad 18$

AUG 7

AUG 7

AUG 7

AUG 7

AUG ? alt. 8000:

$8600^{\circ}$

$6000^{\circ}$

$7250^{\circ}$

$8500^{\circ}$

Sumit $8600^{\circ}$

$8500^{\circ}$

Amanex $8600 \ldots$

7oov ${ }^{\circ}+t$

So00

8500.

$8500^{\circ}$

$8500^{\circ} \mathrm{ph}$ 
1689 Conuuliua

1690

1691. Genothera

1692 Allimn

1693

1691 Panienum

1695

1096

$169 \%$ Croton

1699 Solanum elacagnifolin 1699 Martynia

1800

1801
All 18 San roé Ints. Sonora, Hexicu.

alt. $8550^{\circ}$

Als ; if

"I

$6600^{\circ}$

$6800^{\circ}$

8300

tof San Pedrothevir cace. E.C. nerton 
Solanum elecagmfole

$$
\text { h } 1698
$$

ahs save handwit of labere

$$
\text { Fan } 273
$$

What hilderit 
1689 Conuuliua

1690

1691. Genothera

1692 Allimn

1093

1639 Panienn

1695

1096

$169 \%$ Croton

1698 Solanum elacagnifohim 1699 Martynia

1800

$1 \% 01$
San ise' Mts. Sonora, Hexien.

alt.8550:

Ane $; 18$

Aूध 718

ABu 7

AUS 7

Pohuston's Rauch, "su. East of Sau Pedrothevir cace. E.C. Herton

$6600^{\circ}$

$6800^{\circ}$

8300
AUG

AUG ?

AUG

AUS ?

AUG

AUS i

Aw

Anc 
1802

1803 Ueacia

1801 Tribulus

120 S Parienn

$1 \% 06$

$190 \%$ Amaraintices

$1 \% 09$

1309 a'sfidium Thurberi zuotm. Aus?

1 10 Jacropha

1711

1.1.2 Thasulus

$1 \% 13$ Potutilla

1314

$A !$

AUS 7

Aliti \&
Aus , 18

AUC 71893

AUG ? 1893

AUG 1899

AUE 7

AUG

Aug 3

AUG 8

nachuca Mts, Arzona - (A.X. Holgair)
Shuston's Rauch, IIm. east of San Pedwo Rhior coll.E.C. Herton.

11

"

$1 /$

/I

T 


\section{Qunas}

1816

$171 \%$

$1 \% 14$

1019 fuglans

19.1) Qurens

1821 curbutus

1822

$1 \% 23$

1\%28

1725

$1 \% 26$

$1 \% 2 \%$
AUg $8 \quad 188$

AUG 8 180

AUG 8

AUG 8

AUG 8

AUG 8

AUG 8

AUG 8

AU⿴ 8

AUG 8

AUG 8

AUG 8

AUS 8

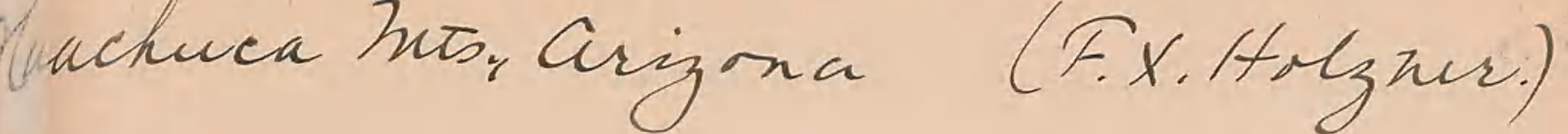




\section{9}

1829

1730

1731

1832

1733 Rhus

1834

1735

1736

1737

1739 Pandocymoptems nontamiso

1739 Oenothera

$1: 30$ Leracium

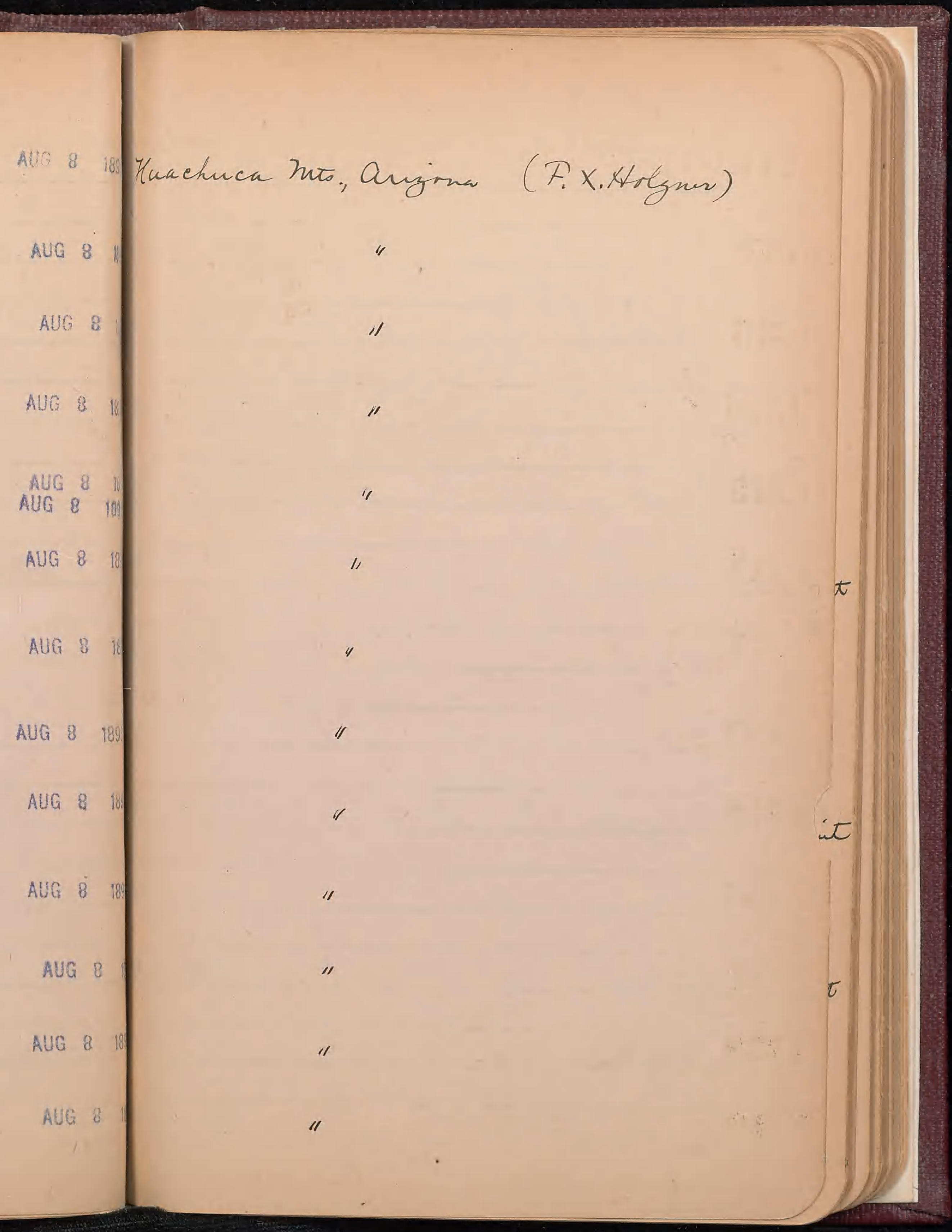


$1 \% 1$ Euphorbia

$1 \% 8$

1743 Commulina

\section{4}

1885 Pulaca

$1 \%$ Cyperus

1789 Comunctima

1895

1819 Eriogrmm

1750

1851 Xenchura

1752 Solamm

1853 Castilleja
AUG 31893

Huachuca Mnt, Arizion - (F.X.Holgur)

AUG $8 \quad$ 189

AUG $8 \quad 1893$

AUG $8.188^{\circ}$

A.lG 10 1898

Sau tosi Wts, Sonora, Hexieo.

AUG $10 \quad 109$

"

AUG $10 \quad 184$

AUG 10 10:

AUG 10

"I

AUG 10

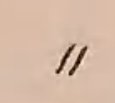

AUG 10

,

ALG 10

AUG II alt. 8600. Sumit

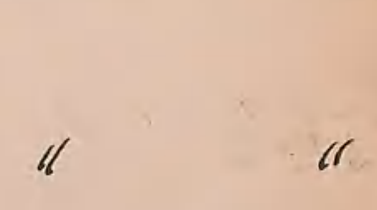

alt. $8500^{\circ}$

alt.8600 - Sunumit

alt-sovo

alt. $86000^{\circ}-$ at sumit

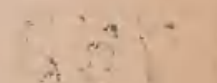


1854 Uschpiar

1755

1856 Qneras

$175 \%$

1755

1759

1760 Phoradindron

1761 Oxybughns

1762 Sefidium

1863 Saunitalia

1864

1765 Enignon

1766
AUG $11 \quad 1893$

AUG II 1893

Augi $11 \quad 1893$

AUG if 1893

AUG $11 \quad 1893$

AUG $11 \quad 1898$

AUG $11 \quad 1893$

AUG i1 189 Thurteri untin Aur 11 is Aur. $11 \quad 1093$ Aus II 1893 AdG 11 AUG 12 108
San fosi Muts, Sonora, Mexico.

alt. $7600^{\circ}$. $a t \tau .6000^{\circ}$ alt. 5550

alt. 5750

alt. Gavo"

att. $6000^{\circ}$

alt. $6000^{\circ}$

alt. $6100^{\circ}$

Galluin fpring act. 6390 .

lear Monmunet 91, huk borud bier. 
196. Aquilegia chryoantha (1torst)alngye 121823 1769

1969 Castillya

1890 Valeriana

$18 \% 1$

$1 \% 2$ Cechillea millefolimu 2

$1 \% 183$ Delphinum

$1 \% 98$

1985 Draba petrophila Grene AUG 12189 1896 ürominum

\section{9}

$18 \%$

$18 \% 9$

\section{ALC 121893}

AUG $12 \quad 1893$

AUG $12 \quad 189$

AUG $12 \quad 189 ?$

Autu 12180

Aur: 12 18:

AUG $12 \quad 18$

AUG 12

Auf 12
AUG $12 \quad 18$

AUG 12189
Tohutowis Rauch, hear how 88. Mux. Bonut lim

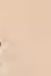


1880 Vicia

1881

1782

1783

1784

1885 Razounof sha

1786 Yiva

$188 \%$ Dotintilla

1785 Yhulypodimu

1.89 Kabuaria

$1 \% 90$

1791

1792 Rhus radicaur th
AUG $12 \quad 1893$

AUG 12 189:

AUG $12 \quad 1893$

AUG $12 \quad 1893$

AUG 121893

AUG $12 \quad 1893$

AUG $12 \quad 189$

AUG $12 \quad 1899$

AUG $12 \quad 189$

Aug 12 18

AUG 12

AUG 12

Aun 12

Whustois Rauch, nar Kiomunut 88, Hux, Bonus

" 


\section{3}

\section{4}

1895 Aerr nugundo $\mathcal{L}$.

1896 Qurens

180: Rubus

1998 Pthea trifoliata 2.

1799 fuglans

1800) Ceauother

1801 Minmelus

1502 Quereus

1803

1804

1805 frucus
AUG $12 \quad 183$ Phuston's Rauch, near nonumut no 88.
AUG $12 \quad 1892$

AUG $12 \quad 1893$

AUG $12 \quad 1893$

Auf $12 \quad 1993$

AUG $12 \quad 1893$

AUC 12 1898

AUG $12 \quad 1899$

AUG 12

AUG 12

AUG $12 \quad 189$

AliG 12

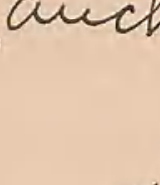

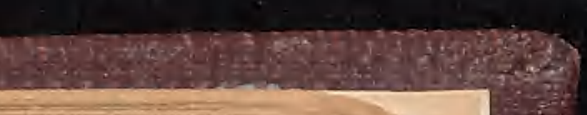




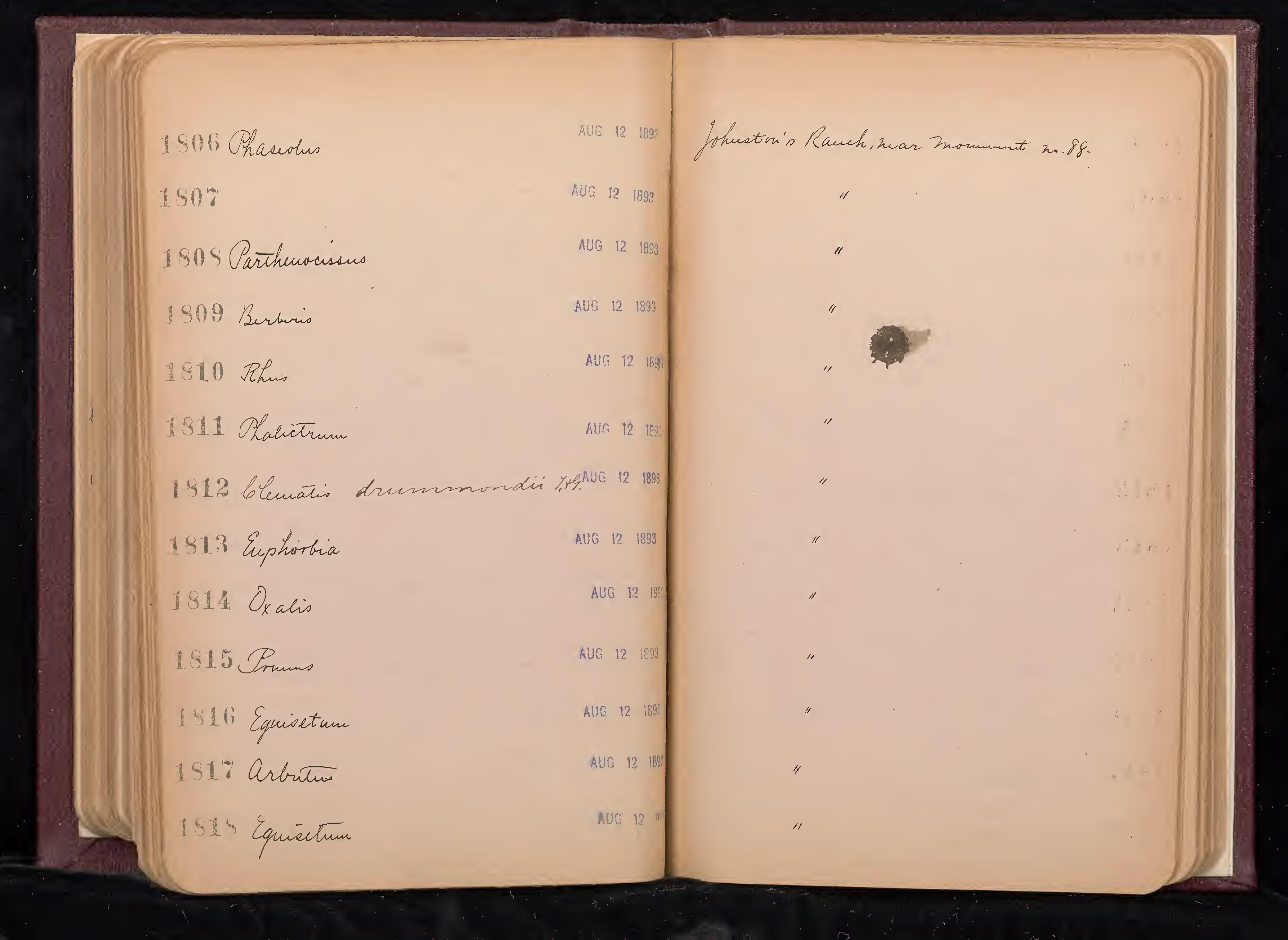


1819 acer

1890

1821. Zeranim

19\%2 Lelematis liquaticifolia tuñ, ANG 12 1893 1825 Mibonia

\section{$19 \%$}

1825 Bouvardia

$15: 6$

$18 \% \%$ Yitio

1325 Solannm

1929 Rentsturon

193 Oenothera

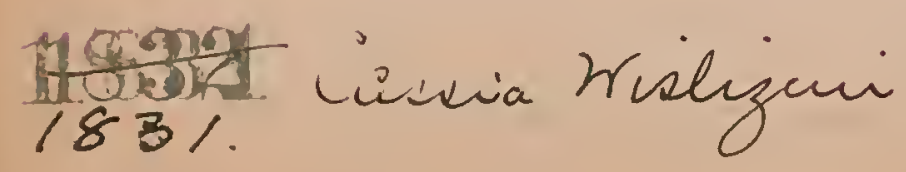

AUG 125283 Aus 12 i8?

4!) $12 \quad 1893$ AUG 12

Aug $12 \quad 1893$

A.J $12 \quad 1893$ AUE 12.889 AUG 12

AUG $12 \quad 1893$

AUE $12 \quad 189$

RUG 12

AUล 13

\section{Shust on's Rauch, zear homunut no. Sg}

"

$"$

Nutch Charly's Pauch suar novmunt \&8. 
1332 iassia

1833

1831 Sanvitalia

1835 Solamun elacagnifolumin

1836 Cyperm

1837 Tribulue

1835 Physalis

1839 Helionia

1840 Dellawa

1841

1812 Acacia

1843

1844 Chunis
ANG 131893 Dutch Charly's Rauch near hoounumut no.88.

AUG 131893

Aus $13 \quad 18$

AUG 13189

AUG 13 189

AUG $13 \quad 18$

AUG $13 \quad 18$

AUG $13 \quad 18$

AUG 13.189

AUG 13

AUG $13 \quad 18$

AUG 13

AUG 13 


\section{5}

1816 Asclepias

\section{1:}

1815

1919

1850

1851 Kotholauna

1852

1953 Cypodanthera

$195 \%$

195.) Portulaca jillosa

1956

$185 \%$

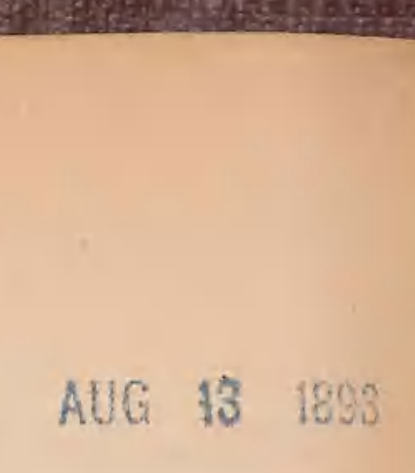

Jutch Chardy's Rauch, muar Homumut in 88 


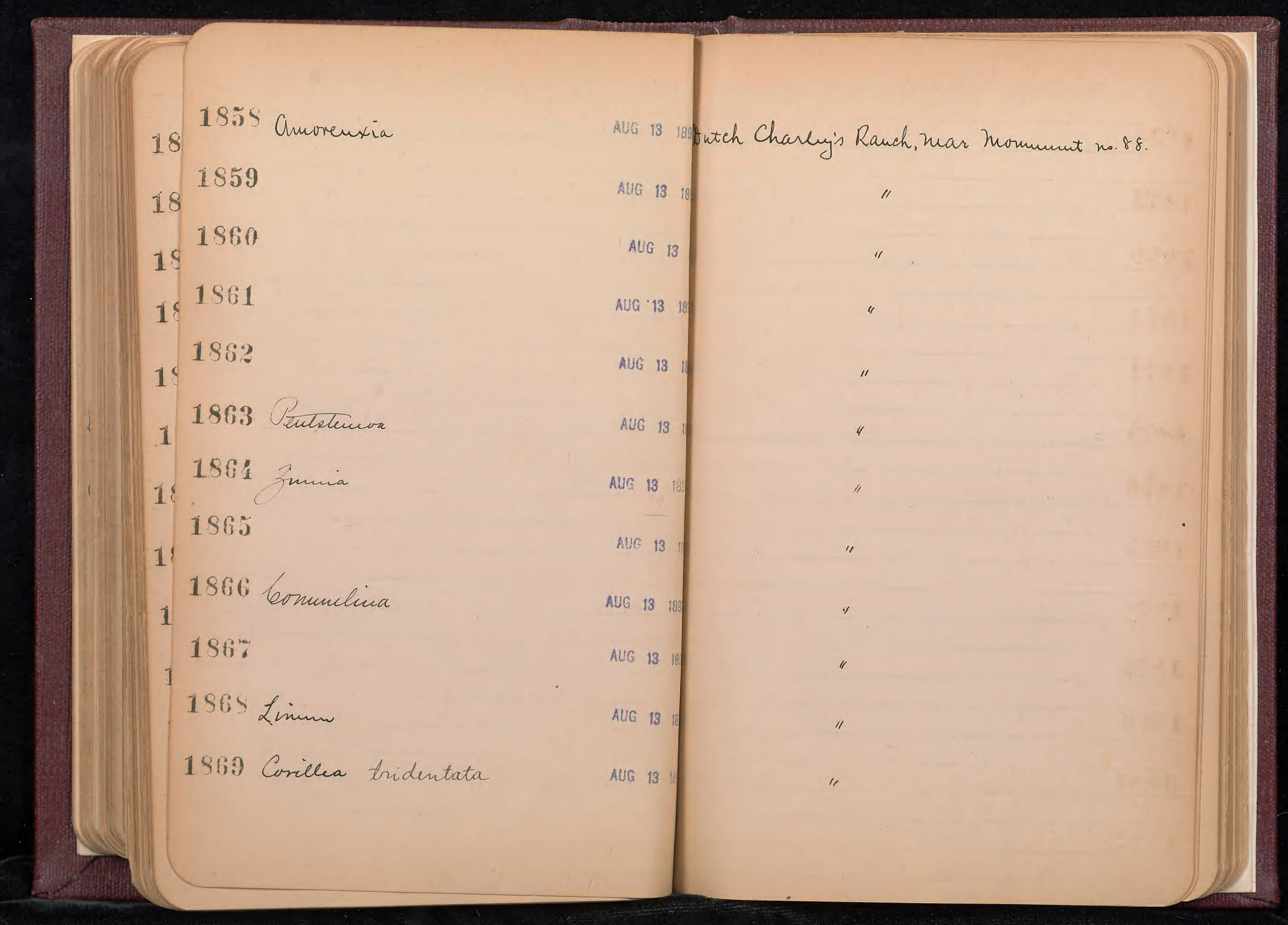




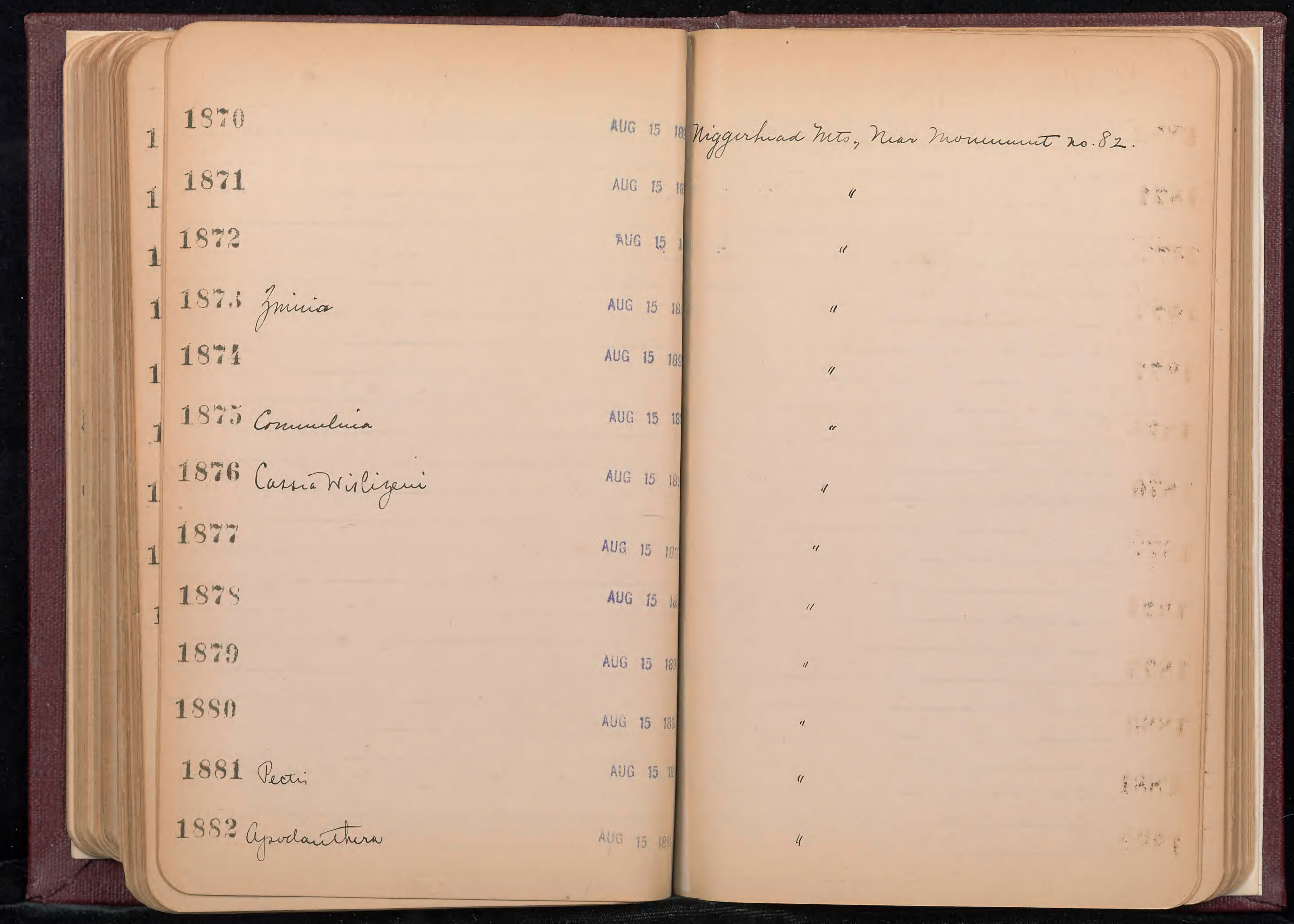


$1996 / 20$ ourdia

$1599^{\circ}$

1995

1999

1909 Peutoteruon

1901

$190 \%$

1903

1901 Yerbeua

1905

1906

$190 \%$

1905
AUG $15 \quad 1893$ Nigguhead Wuts. Wear Hounumut no 82

AUG $15 \quad 1893$

hug $15 \quad 1893$

A.te $15 \quad 1893$

AUG 15 isi

Alic $\quad 15$ i893

AUG $15 \quad 18$

Alle $151890^{\circ}$

AUG 15.19

ADG $15 \quad 191$

AUG $15 \quad 18$

430 if 18

AUG 
1909

1910

1911

$191 \%$

1913

$191 \%$ Virbena

1915 Sanvitalia

1916

$191 \%$

1919

1919

1920

1921
ALG IT 1993

A $6317 \quad 1893$

Aug $17 \quad 1893$

43017

AUG $17 \quad 1893$

NuG $17 \quad 1893$

AUG $17 \quad 1893$

AUG if 1893

AUG $17 \quad 1893$

$.40 G \quad 171803$

Aug $17 \quad 103$

AUG $17 \quad 1993$

Rutio if 1893 Niggrhead Nuts, hear Konumut no.83.
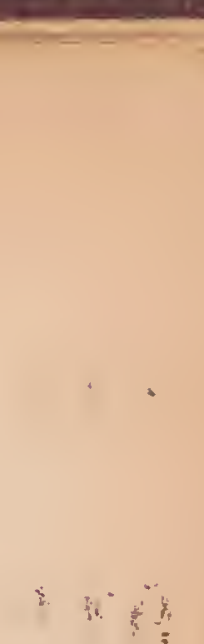
1922 Pentotumon

$19 ? 3$

1921 Eniogormun

192573 oururdia

1926 Lponvea

$19 \%$

1935 Querens

1929 Solanmu

1930

1931

1932

1933 Solarum

1931
Alg $17 \quad 1893$

$806 \quad 17 \quad 1893$

AUG $\quad 17 \quad 1893$

AUG $17 \quad 1893$

AUG $1 T \quad 1893$

AUG i7 1893

AUG $17 \quad 1893$

AUG 171893

Nug $17 \quad$ is?

AUS $17 \quad 1893$

AUG $17 \quad 1893$

AUG $17 \quad$ 180

AUG $17 \quad 1893$
Tiegurhad Tuto, hear Monumut ns.82 Tigerlo

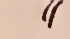

4

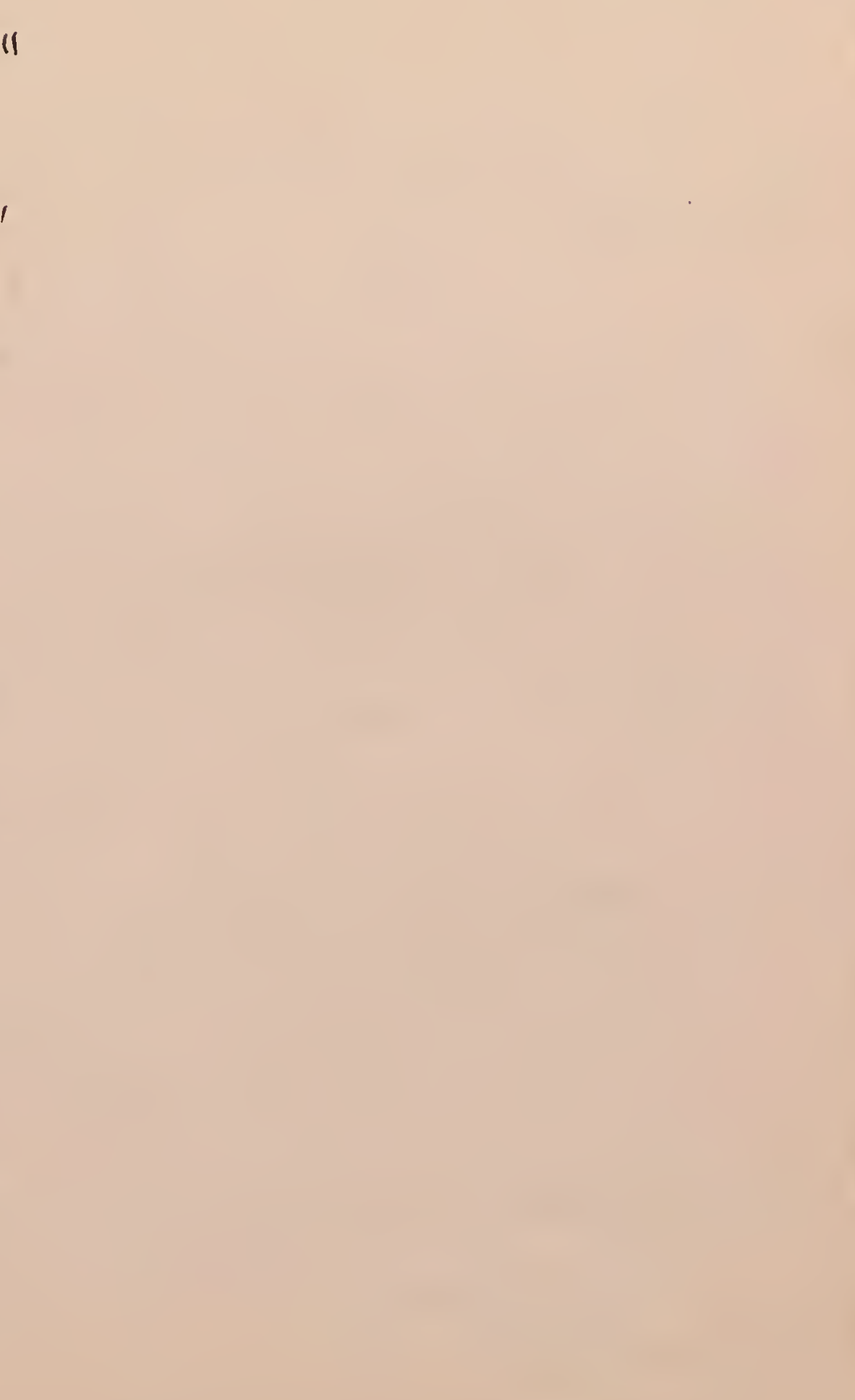




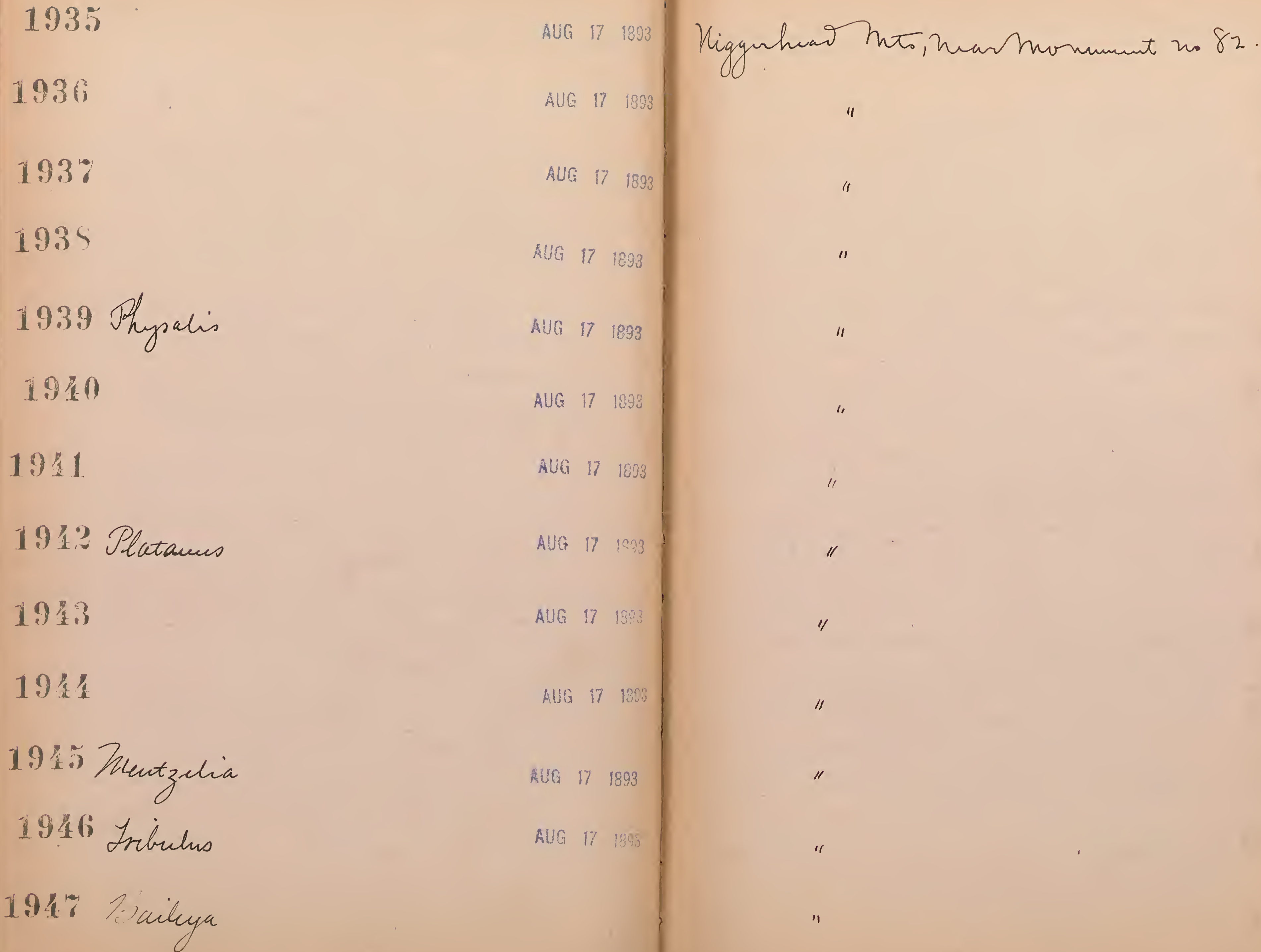

1935

1936

$193 \%$

1935

1939 Physalis

1910

191.

1912 Platanes

1939

191

1915) Dentzelia

1916 Iribuhs

194 Tiaileya

AUG $17 \quad 1893$

AUG $17 \quad 1893$

AUs: $17 \quad 1893$

AUG $17 \quad 1893$

AUG it 1893

AUG $17 \quad 1893$

AUG IT 1893

AUG $17 \quad 1003$

AUE $17 \quad 1803$

AUc 17 1000

स⿺𠃊 $17 \quad 1893$

AUG I7 9005

Kigguhuar thto, hav Monmunt no 82 .

$"$

$" 1$

11

'"

"

"I

is 
19积

1919

1950 Tifolciun

$195 \%$

19.5\% Iponvea

1953 Euphorbia

1954

1955 Eryngnim

1956 Salnia

$195 \%$

195s Delphimin

1959 Oenoctura

1960
AUG $20 \quad 1893$

AUG 201898

AUG $20 \quad 1893$

ALG 201893

AUG $20 \quad 1093$

ALS $20 \quad 1893$

AUG $20 \quad 1893$

AuG $20 \quad 1803$

AUG $20 \quad 1893$

AUG 20,1893

sevfularimaltray AUG 211893

AUG 211893

AUG 2ब1 Thog

Huachuca hits, Ariz-(coll.F.X.Holgner) 


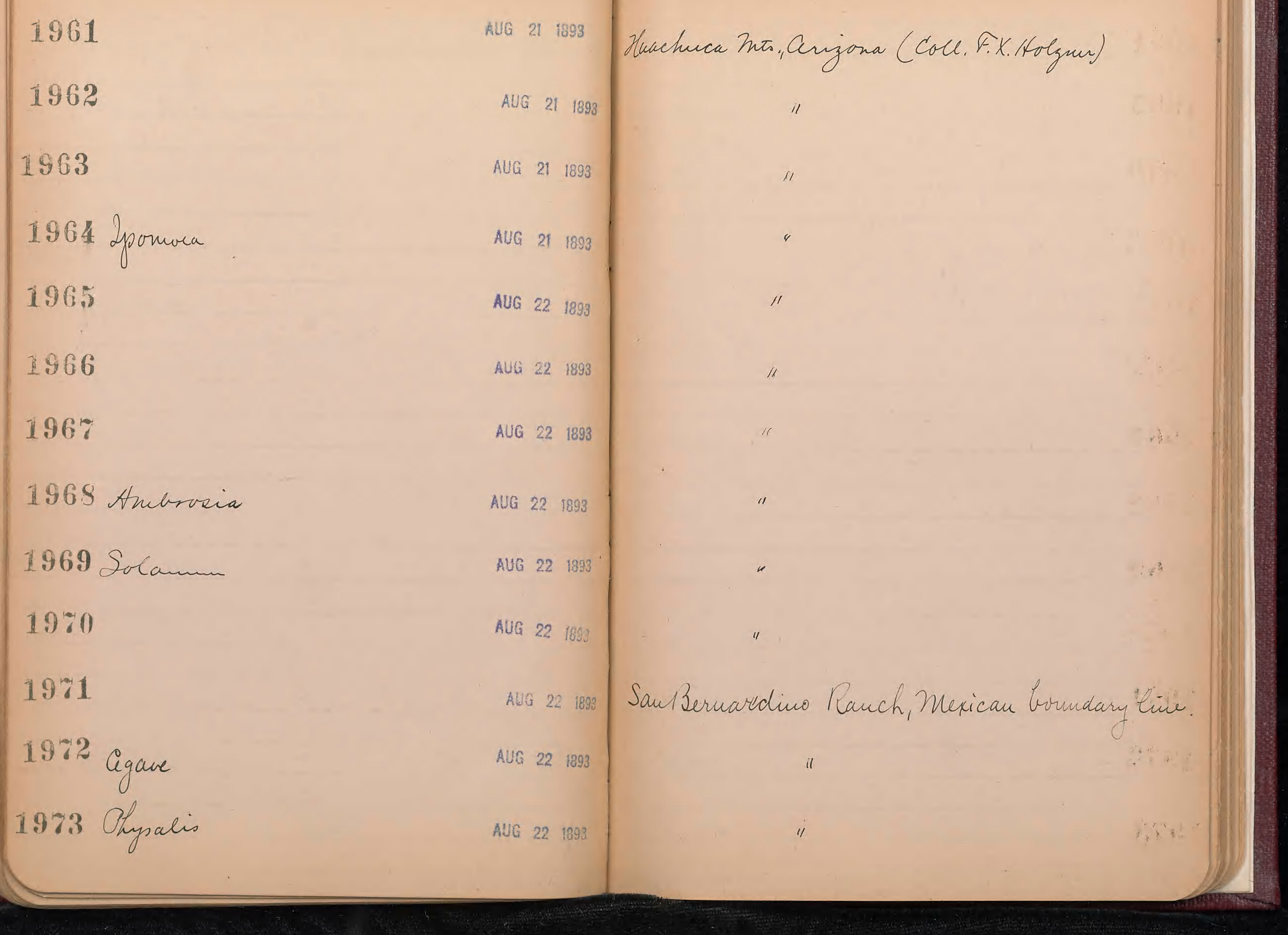


1984

1985

1996 Cypan

$19 \%$

19 's Eleochain

1989

1980

1981 Euphorbia

1982 Tribulus

1993

1954 Snbulus

195.

1886 Salis
AUG 221899

AUG $22 \quad 1893$

AUG $22 \quad 189$

AUG $22 \quad 1893$

AUG 221893

AUG $22 \quad 1893$

AUG $22 \quad 1893$

ALC 221898

AlJG $22 \quad 189$

Allo $22 \quad 1803$

AUG $22 \quad 1898$

AUG 221803

AUG 22 189:
San Bernardino Rauch, Muxic an boundarytim 


\section{$198 \%$}

1985 Cucurbita digitata

1989 2ponoca

1990

1991 Cacsulpinia

1992 balix

1993 Carduns

1994 2ponola

199.) fomoca

1996: Cassia fyptarpa Bunth.

$1999^{\circ}$

1995

1999
AUG $22 \quad 1893$

Sau Beruardino Rauch, Muxicau boundary lim

AlG $22 \quad 1893$

AUG $22 \quad 1893$

AUG 221898

AUG $22 \quad 1893$

AUG 22, 1893

AUG $22 \quad 1893$

AUG $22 \quad 1893$

AU6 $22 \quad 1893$

AUG $22 \quad 1893$

AUG $22 \quad 1898$

AUG $22 \quad 1898$

AมG $22 \quad 1893$
$9 n$

W

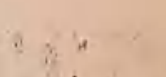

${ }^{7}, 0$ 
2000

2001 Argluon

2002

2003

2001 Bailya

2005 Datura neteloides

2006 Amarawehs

200' beyperus

2004

2009

2010

2011

201:
AlG $22 \quad 1893$

AMG 221893

Aง 221893

AUG 221893

$40622 \quad 1893$

AU⿴囗十 221833

AUG 221893

Aug 221895

Alu $23 \quad 1993$

(1)

AUS 20

AUG $23 \quad 1893$

A U⿺ 23 :

AL $230=25$
Saw Bermardino Pauch, Muxican boundary lim.

"

lir

, 1

"

"

Unachuca Wits, Arizona (coll.F. X. Avolgnes) 
22013 2ponvea

22014

2 2015 Erymginum

2016 Tradescantia

2 201: Comunelina

22015

2019 Enphorbia

$.202 n$

2021 Cuncucarpus

2022

2023

2024 Arturisia

2025
AU: 23180 Wuachuca Whts, Arizona (F.X. Holgur)

AUG 23

AUG 27

AUG 24

AUG 24

AUG $25 \quad 18$

AUG $25 \quad 189$

AUG $25 \quad 189$

AUG $25 \quad 189$

AUG $C B \quad 1893$

AUG $20 \quad 189$

AUG $26 \quad 180$

AdG $26 \quad 18$
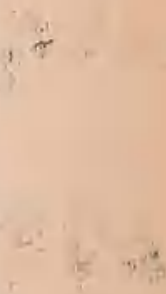
2. 2026

2 202i Rhus

22025

2. 2023

: 2030

2031

203: Physalis

2033

2031 Pctis

2035

2036

$203 \%$

2035 hibomia
AVG 261093 Huachuca Wuts, Arizina (F.X. Holgmir) AUG $26 \quad 19$

AUG $26 \quad 1893$

AUG $27 \quad 1893$

AUG $27 \quad 1893$

AUG 271899 Ynadulugse Cacion, Sonora (EC.Murton)

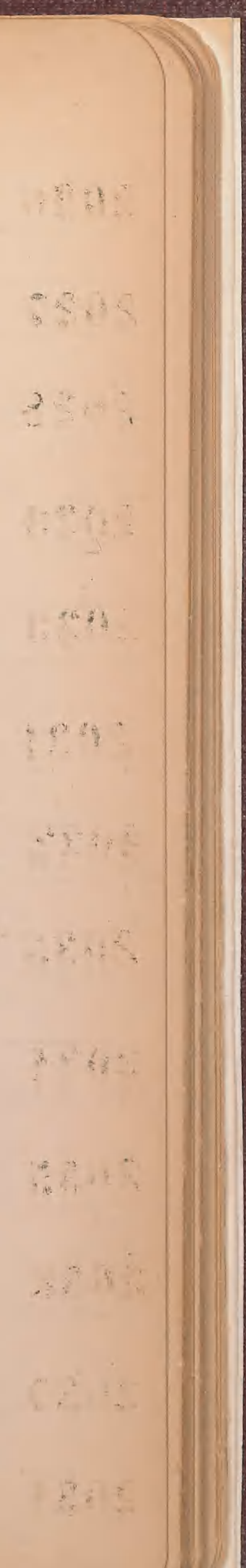


- 2039 Nuciboniá

: : 2010

2 2011 Sanvitalia

2012 Yerbuna

2013 Salvia

2031

: 2015 Bverhavia

2016 Eriogoum

$20 \%$

2015 Pecti

2019

2050 Cyperms

2051 2pomoer

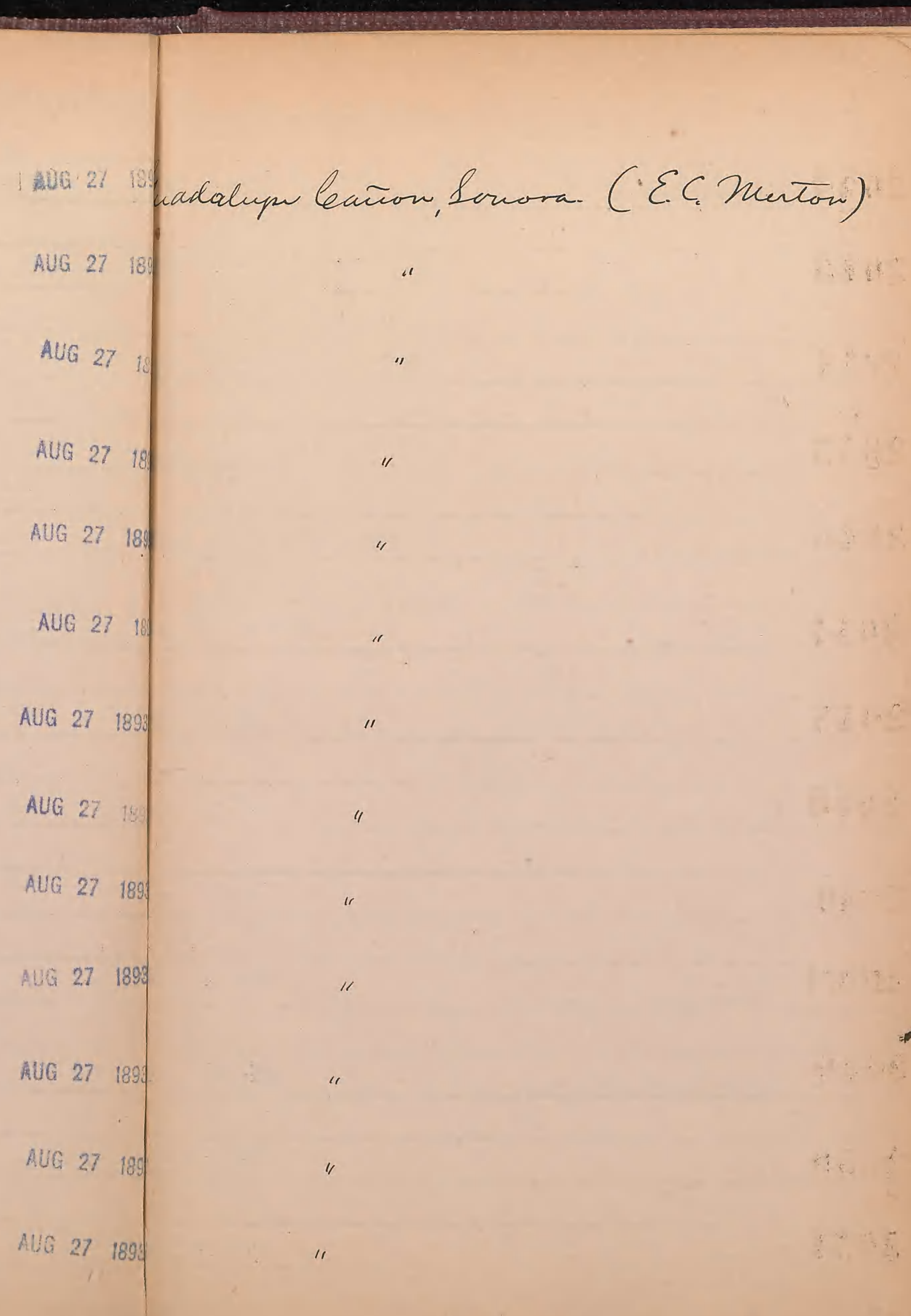


2052 Mirabilis

2053

2054

2055

$2050^{\circ}$ Querens

$205:$ Platann

2055

2059 Lathyrus

2060

2061. Phasulus

2062 Mafniowizia

2063

2064 Salmi
AUG $27 \quad 189$

AUG $27 \quad 189$

AUG $27 \quad 1893$

AUG 2.71893

AUG $27 \quad 1893$

AUG 271898

AUE $2218 \%$

AUG $23 \quad$ 193

ALG $23 \quad 100$

AUG $23 \quad 1980$

AUG 231093

AUG 23 in

AUG $28 \quad 10$
Inadoluyse Cacion, Sonora (E.C. Merton)

"

"1

Huachuca net. Arrzina (F.X. Aolgur)

Zuadahpe Cauon, Sonora (ECDurtoa) 


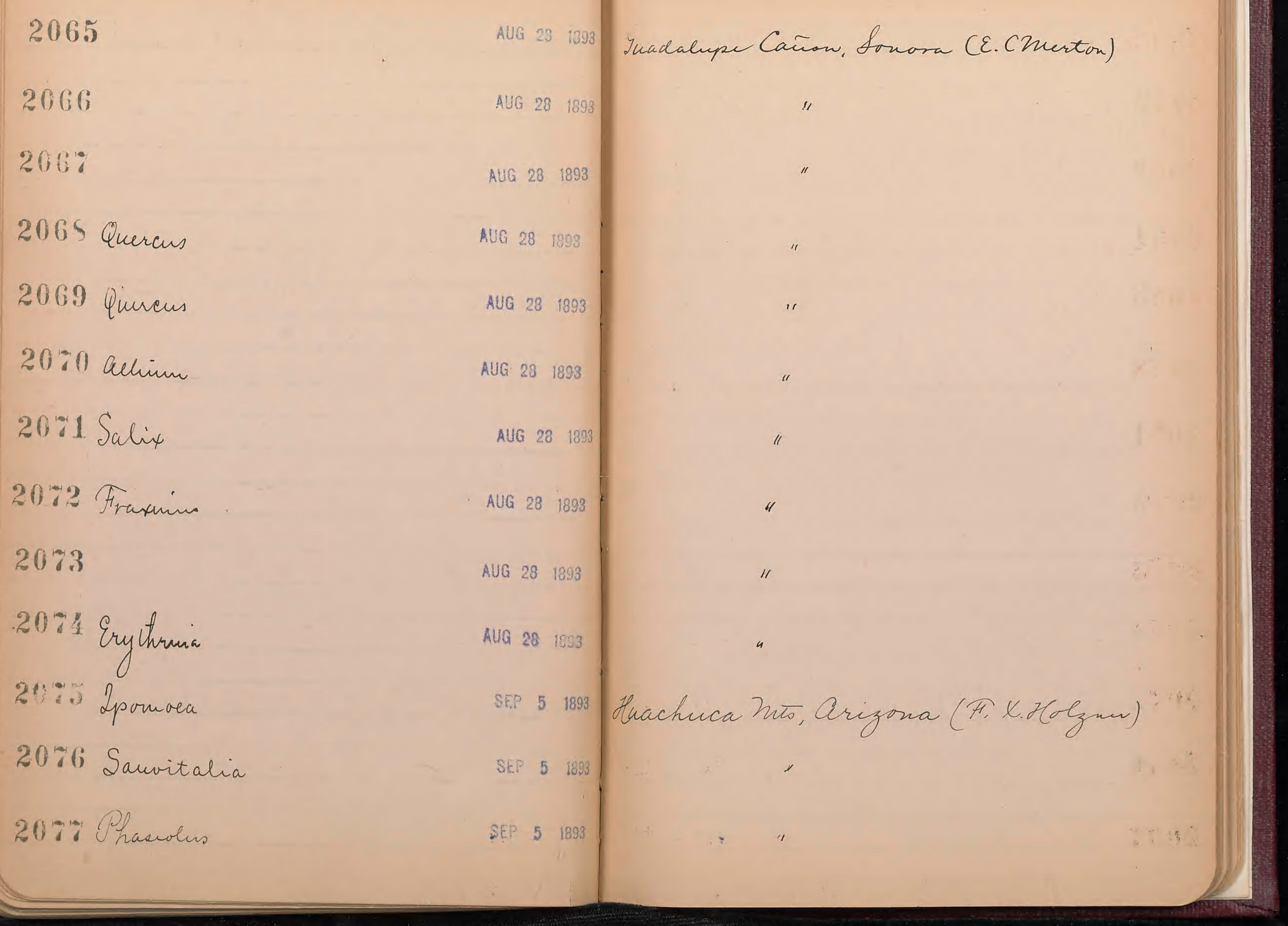




\section{0 is Mibonia}

2079 Pavicun

2080

2081

2082

2083

2081 Erigounm

205.) Eriocarpun

2086

$208 \%$

20:S Mollugoberticillata 2 2089

2090
$508 \quad 51893$

StP 51898

SEP $5 \quad 1893$

SEP $5 \quad 1893$

SEP 51893

SEP $5 \quad 1893$

$547 \quad 5 \quad 1898$

SEP $\quad 5 \quad 1893$

SE $5 \quad 1893$

SEP 51898

$\operatorname{sen}^{2} 51893$

Sts $-5 \quad+1843$

SEP 51893
Xnachues hot, Arigona (F.X. Holgners)

Base fo tandinis nits, up $\$$ bovofy. 


\section{1}

2092 Carduns

2093 Panicinn

2094

2095

2096 Erugrostis

$209 \%$ Eragrostis

2095

2099

2100

2101 2xophorm

2102

2103 Lida Base of Saw hus hets. up to 6000 .

5 1893

SEP $\quad 5 \quad 1893$

SEP $5 \quad 1893$

SEP 5 160

2 $5 \quad 1893$

SEP $5 \quad 1998$

SEP $5 \quad 1893$

SEP $5 \quad 1893$

SEP 51893

SEP $5 \quad 1893$

SEP $5 \quad 1293$

SEP $\quad 5 \quad 1893$ 
2104

2105) Bonteloua

2106

$210 \%$ Aritida

2105 Amarauther

2109

2110

2111

2112 Peutotenon

2113

2114 Autirrhimum mauraudioider 2115

2116
SE? $5 \quad 1893$ "3anu of San Luis his, up to loovo:

SE $5 \quad 189$

SEP $5 \quad 1993$

SEP $\quad 5 \quad 1893$

SEP $5 \quad 1893$

SEP $5 \quad 1893$

$\begin{array}{lll}S E P & 5 & 1893\end{array}$

SE 51893

$865 \quad 5 \quad 1893$

Ste? $5 \quad 1893$

Jep $5 \quad 1893$

SEP $\quad 5 \quad 1893$

SEP $\quad 5 \quad 1893$
4

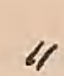

,

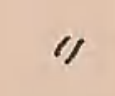

/,
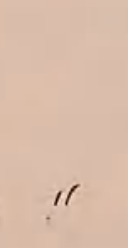
211:

2115

2119 Muibonia

2120

2121

2122

2123

2121 Phaseolus

21:5 Monarda

2126 Filia

21?:

2129

2129
- 51993 Base of Sau Leis Wuts, up to 6 ovofr

$65 \quad 5 \quad 1893$

$\operatorname{sen} \quad 5 \quad 1893$

SEP 51893

$S E 51893$

$\operatorname{sep} 51893$

$\operatorname{sen} \quad 5 \quad 1893$

889898

$S L P \quad 5 \quad 1893$

SE $5 \quad 1893$

$3 E^{2} \quad 5 \quad 1893$

SEP 51893

SeP $5 \quad 582$
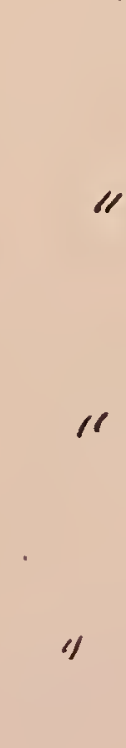

"

"

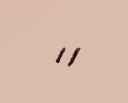


2130

2131

2132

2133

2135 Aenothera
2134 beperus

$50 \quad 5 \quad 1893$

SE: 51093

SEP 5 - 180

SE 5

$56 \quad 5 \quad 1893$

SEP $\quad 5 \quad 1393$

2136 Celematis

$213:$ vastillya

2135

2139

2110

2111 Eleocharis

21:3 Eranium

\section{liquaticifolin nuty, SEP 5}

$3 P^{2} 51893$

SEP $5 \quad 1893$

$S^{3} \quad 5 \quad 1893$

$\begin{array}{lll}S E P & 5 & 1893\end{array}$

SEP 51893

St: 51893
Base of Sam Lair huts up to $6000 \mathrm{fth}$. 
2113

2111 Cyperus

2115 vtascolus

2146

2118 Janchunia

2115 learduns

2119

2150

2151 Peutstemon

215: Inaphation

2153

2151 Crotaluria

2155 Firainlís
Sep 51893

SEP $5 \quad 1893$

Base of aw Lin the up theovoft.

SFP $5 \quad 1893$

SEP $5 \quad 1893$

SEP $\quad \begin{array}{ll}5 & 1893\end{array}$

SEF $\quad 5 \quad 1993$

SEP $5 \quad 1893$

SEP 51893

SEP $\quad 5 \quad 1893$

$\begin{array}{lll}\text { SEP } & 5 & 1893\end{array}$

SEP 51893

SEP $5 \quad 1893$

SEP $5 \quad 1893$ 
2156

$215 \%$

2159

2159 Eriquon

2160

2101. Drlphinim

216? Pentitemon

2163

2164

2165 ricia

2166

21.:

2165 allium ase f Sanduis hito up to boorft.

SEP 51893

SEP $7 \quad 1898$

SEP 71893

SEP 71893

machuca hits, Ariz -(F.X.Holgner) 
22169

2 $21 \% 0$

$221 \%$

2218 Mebus

2183 Thalictrum

2213

22195 Heuchera

$221 \% 6$ Salix

$221 \%$

$21 \% 5$ Sedme

2189

2.2150

22191

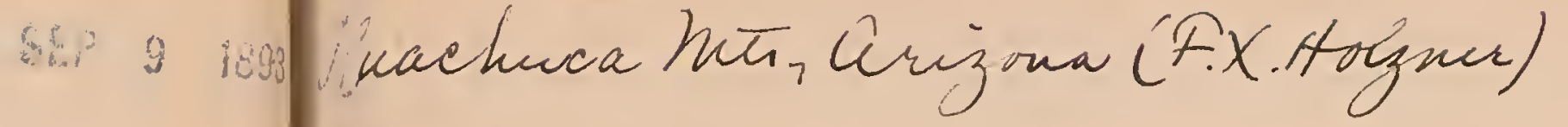

$\sec 9$

SEP 9

SEF $9 \quad 18$

$\operatorname{sep} \quad 9 \quad 189$

SEP $9 \quad 1899$

SEP $9 \quad 1893$

S5P \& 189

$\sec 9 \quad 983$

$\operatorname{sen} 1018$

9010

$\operatorname{sep} 10$

$\operatorname{SeP} 10$

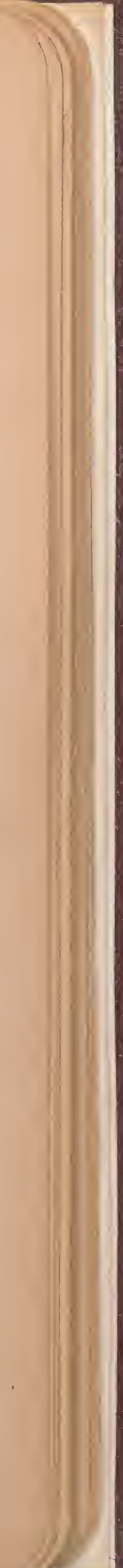


2.8182

- 2183

2184

2. 2185 Pinus

22156 Gimuia

2 $215 \%$

22155

22189 alis

\& 2190 Pellava

2 2191 bheilanthes

219?

2 2193 Sahiun

क 2191 Jeramin

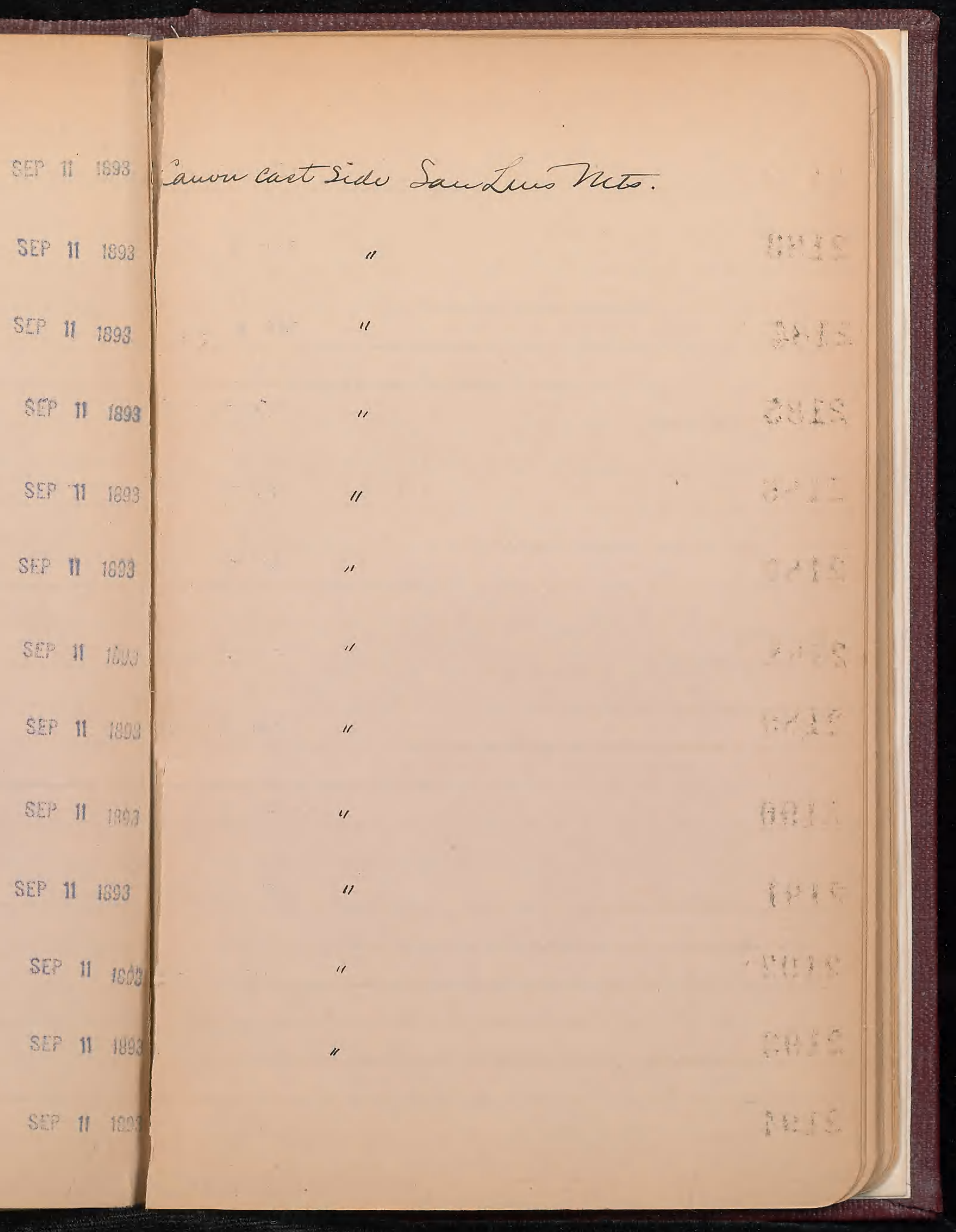


2 2195 Vivila

2196

219: Zaunchuria

2195 Lpomoca

2199

2200

2201 2ponvera

2 2202

2203

22201 Ohyalis

2205 Salvia

2 2206 Drata petrophila Trune $220 \%$

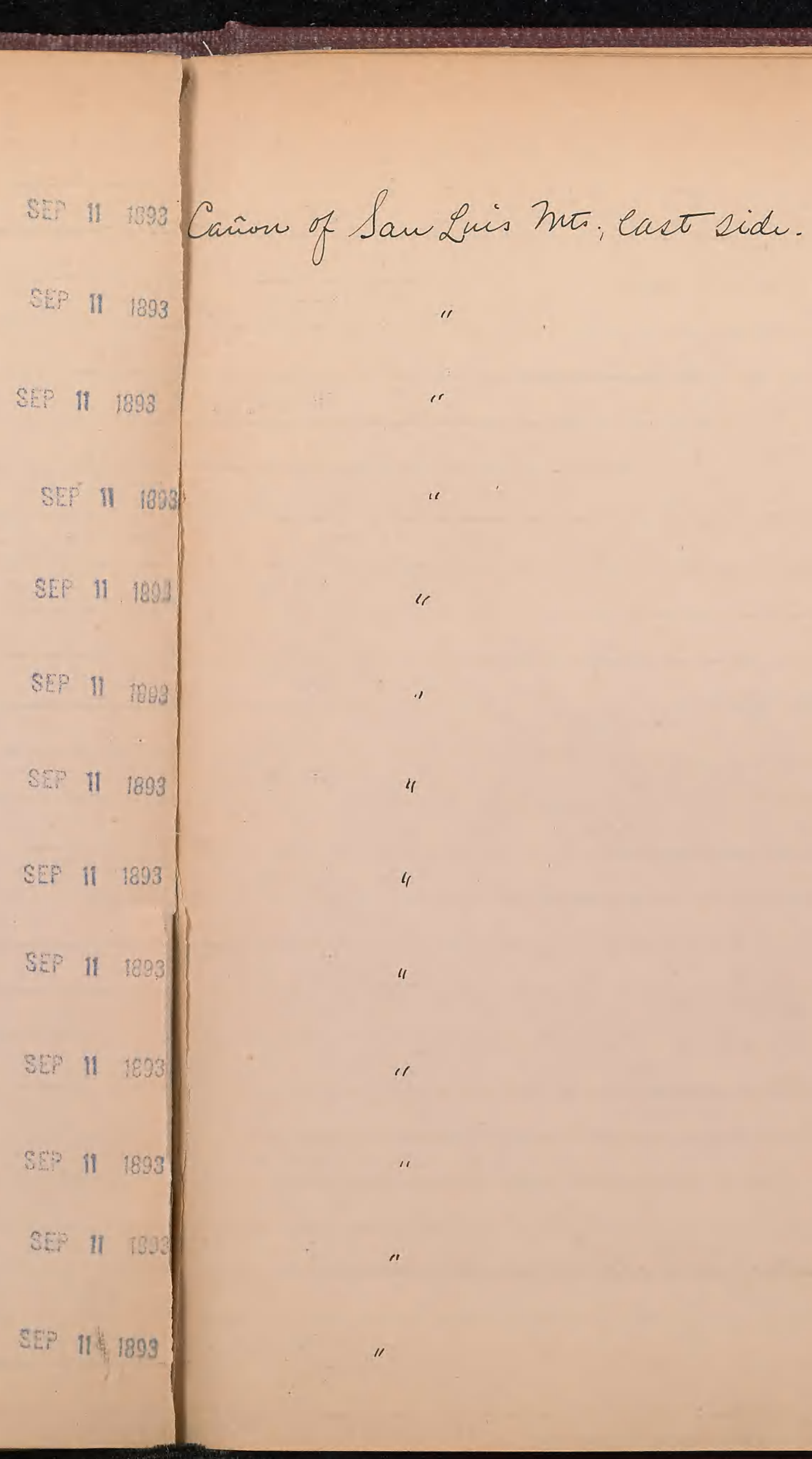


2005 Castillya

2203

2210

2211

$221 ?$

2.213 Cercocaypus

2211 Qunens

2215. Rubus

2016

221\% 2 minu

2215

2.219

2220
11 1893 baũm east sidu Sou Luis Wut.

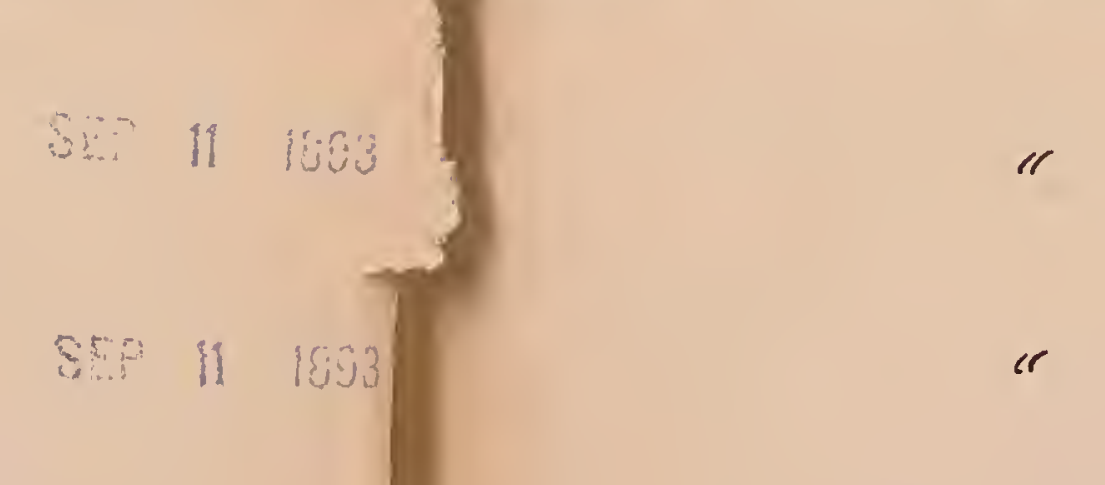

Sef $11 \quad 1000$

$8981:$

Sep 11

85 in 1903

$8 n ; n$

ser it hou

$S 0^{3} 11$

set 11 in

"

$5+11 \cdots$,

sec 1110 


\section{1}

\subsection{Pentrtunow}

2223 Cheuopodiun

2224 Iradencautio

2225 bonumlina

2226

222:

2225

2229

2230 Quercus

2231 Eniggonum

2232

2233
111893 Gaîon east side San Luis Into,

SE 111053

S:? $11 \quad 1393$

SEP II 1893

SEP $11 \quad 1893$

SEP II 1803

SEP II 1893

G.. $11 \quad 1893$

SEF II 1993

SEF $11 \quad 189$

SEP II 189

SEP II 1893

SEP II 1893 


\section{3}

2835 Oxalis

\section{6}

2389

2234

zozs9 Iileue

289

221

2.28

223

2214

$22 \%$

23:10 Quercus
5251893

baĩon east sidu Jau Luir Huts.
SE? II 1893

$\operatorname{sen} \quad 11 \quad 1893$

:SEP II 1393

58 11 1893

88 il 1003

$485 \quad 11 \quad 30$

S. $11 \quad 1003$

SEF $11 \quad 1803$

sep $11 \quad 1003$

SEP $11 \quad 823$

$\sin 11 \sin$

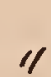

"

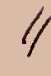

II 


\section{$224 \%$}

\section{5}

2219 Arctostaphyloo

2250

2.2:1

2252

2253

2254

2255 Pantsteurom

2256 Pinus

225:

2255

2259
SEP II 1893 baĩon cact side San Luis heto.

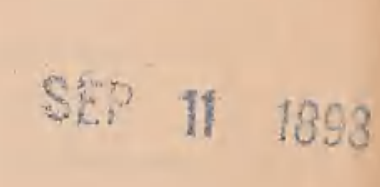

SEP $11-1993$

SEF $11 \quad 1893$

SEP II 1893

sef $11 \quad 1803$

SEP 111390

SEP II 18

SEP II

- SEP If 1893

जE? $11 \quad 1893$

Mexicair boundary liue near White Water 


\section{0}

22131

2262

2263 beyperus

2284

2265

2260

$226 \%$

2265

2269 Croton

22.91

$22 \% 1$

$282:$
(1) 1803 Wexicau boundary lime war Whit Water.

$\begin{array}{lll}\text { SE: } & \text { I1 } & 1393 \\ \text { sep } & \text { I1 } & 1393\end{array}$

85111498

SE? $\| 11893$

ses 11 icos

Sin $11 \quad 1393$

Shy $11 \quad 1993$

$85+11 \quad 303$

Sher $11 \quad 1893$

SE $11 \quad 883$

aEY $11 \quad$ i893

at: 11893

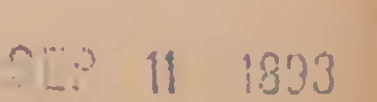


2273 Erugrostis

2284

2285

$22: 6$ Cucurbita factidisimia

$228 \%$ Carduns

$22 \%$ rerbua

2279 Eriogonum

2250 Anoda

2281

2252 Cyperms

2283

2254 Lejidium

2295
SEP II 1898 Mexican boundary live near White Water

SEP II 1893

ste 111893

Sep II 1898

Set $11 \quad 1823$

SEP ก1 1893

SE.P $11 \quad 1893$

SEF $11 \quad 1893$

setp II 1893

SEP $11 \quad 1893$

SEP II 1993

SE? II 189?

SEF $11 \quad 1890$ 
2256 Chunpodium

$229 \%$ Exiogonum

2285

2289 Cyperus

2290 Berlaudira

2.291 Eurotia lauata

2292 Crotalaria

2293 Eriquron

2294

229.) Erivcarpun

2296 cister

229: aster

2299
58. II 1893 Mexicauboundary him near White Water

SEP $11 \quad 1893$

SEP 11

SEP $11 \quad 189$

Exp If 1893

Sto II

SE? $11 \quad 1003$

sop $11 \quad 1893$

SEP if 1093

कहसे II 1993

SEP $11 \quad 1893$

SEP II 1893

St? 11
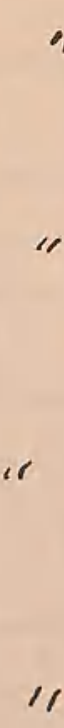

II

II

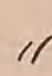

"

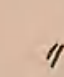




2299
2300 Erynguin
2301
2302 Rumex
2303
2304
2308
2306
$230 \%$
2309
2309 Eragrosti
2310
2311 Eragrootis

SEP If 1893 Mexican bonudany hin, mear White Wath. 


\section{2}

2313

2314 Silen

2315

2316

231 Plantago

2318

2313

2320

2321

2327 Quercus

2323

2321
3EP II 1893

SEP II 1893

Lept .12.1893

Eyp.12.189

$\operatorname{sep} 13.1893$

Lep.14.1893

$\operatorname{bep} .14 .187^{3}$

SEP 101898

Dog Springs, Dog Whts hew Mux. Muxican borudary him near White Water-

Huachuca Ints, Ariz-(F.X.Holgner) $\sec 16 \quad 189$

SEP $16 \quad 1893$

ifp $16 \quad 1993$

SEP $16 \quad \operatorname{lgg}$

ste 16 198 
2 2325 Proposis juliftora

22326

2 $232 \%$

2.232S lorillua tridentata

22329

2330

2. 2331

2 2332

2333

22334 Daibun

22335 Eriocounum

2. 2336 Croton

2 233 ?

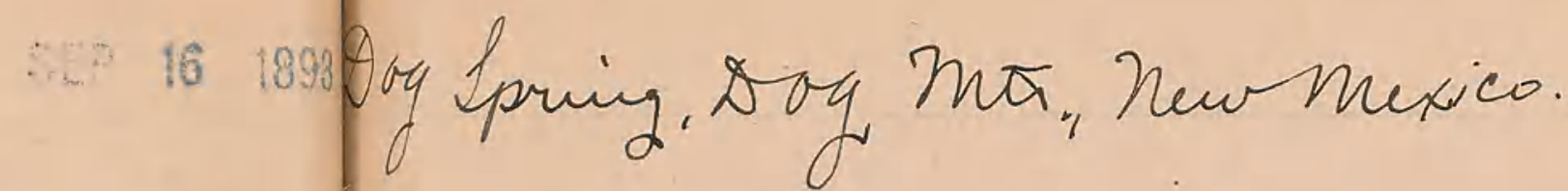

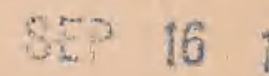

SEP 16

sip 16

Se? 16

$8+9 \quad 16$

SEP 16

$8=0 \quad 16$

S5? 16

SEP 16

SEP 16

SEF $16 \quad 18$

SEP $16 \cdot 18$

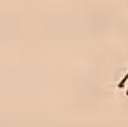

"I 
¿335 Pectis

2339

2310

2311 Eragrostio

2332 Eriogoumn

$: 2333$

2314

2335

2336 Erayrostio

2319

2349

2389 Comminina

2350

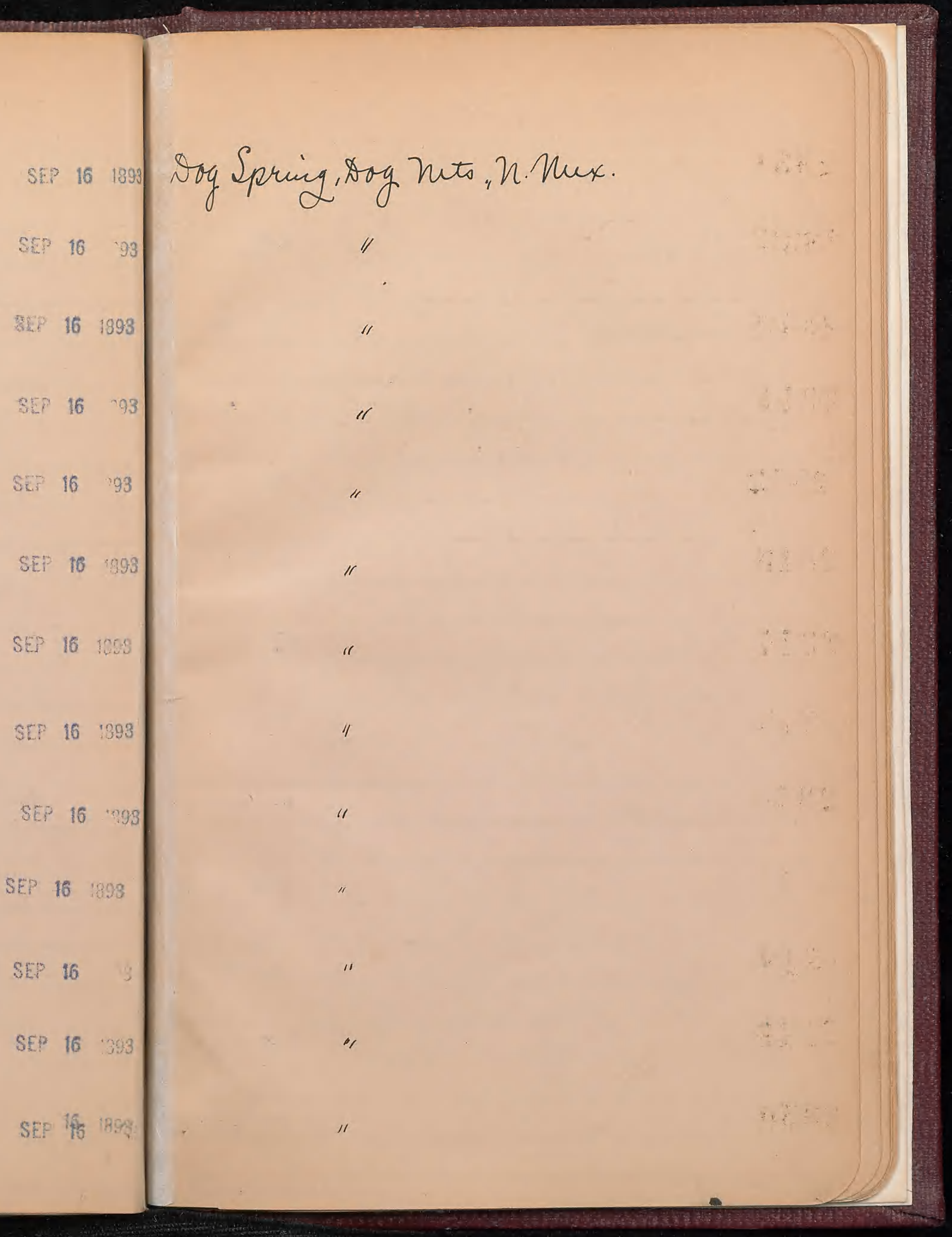


2351

2852 Physalis

235\% Peutstunon

2354

2355

2356

$235 \%$ Carduns

2359

2359 Mirabilis

2360

2361

2362

2363 $\operatorname{stp} \quad 16 \cdot 93$

SEP $16 \quad 1899$

SEP $\quad 16 \quad 1098$

$\begin{array}{lll}\text { SEP } & 16 \quad 1899\end{array}$

SEP 16

$\begin{array}{lll}S E P & 16 & 993\end{array}$

SEP $16 \quad 299$

SEP $16 \quad 1399$

SFP $16 \quad 799$

SEP I6 1899

$=$
SEF

SEP $16 \quad 1898$

SEP $16 \quad 18$
Dog Spring, Dog nets, Nur nuxico. 
2364

2365 Inaphalium

2366

2368

2365 Hedeorua

2369

$23 \%$

$23 \% 1$

2312 Eriogonum

283

$23 \% 1$ Peacicia

$23 \% 5$

2386 tog Spring, sog mi, n. Mex. 
$237 \%$

$23 \% 9$

2389

2380

2381

2382 bastilleya

2383

2384

2385

7286

$238 \%$

2389

2389 Maxmoviczia
SEP 17 Dog Lpring, Dog Mnts, Neur Mnexico.

sep $17 \quad 189$

if

at? $17 \quad 13$

SE $17 \quad 1899$

SEP 17189

SEP $17 \quad 189$

Sep $^{\circ} 17 \quad 893$

SEP 17 189:

SEP 17

sep $17 \quad 199$

stip $17 \quad 893$

SEP 17 is

SEP $17 \quad 1893$ (2sheets nomuta) 
2390

2391

2392 Nuhiustura

2393

2391

2395 Phoradeudron

2396 Quercus

$239 \%$

2395

2399

8100 Pectis

2401

2102
Dog Lpring, Dog Huts, Hew Merico. 
2403

2404

2805 Cestrugalus

2406 Sauvitalia

$210 \%$

2409

2409

2110 Amarauchus

211

2113 Polygonum

2113 Solidago

2111

2115
SEP 17,923

Dog foring, Dog Hot, Nus Mexico. sep 17

SEP $17 \quad 189$

SEP 17

SEP 189

$\sec 27189$

Supt. 22.189

Sep 22. $189^{\circ}$

SEP $22 \quad 180$

SEP 22. 1893

\begin{tabular}{lll}
\hline 5 & 22 & 1893
\end{tabular}

as? $22109 \%$

SEP 22
"

"

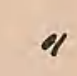

")

" 
2110 Eriguron

2:1: Populus

215 Xantium

2119 Salix

2120

2121

2220 bonopholis

2.23 Aiter

$2: 21$ Solidago

212\%Salix

2156 Araclichia

2:2' Phoradindron

2134 Sotidago voy Spring, Sog Nit, Nuxido

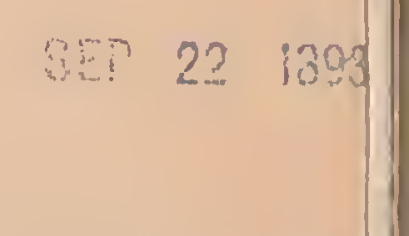

36227893

22.1803

$\operatorname{sen} 22.1803$

80122.1992

3221893

SEP $22 \quad \operatorname{lng} 3$

set 221699

215 2?

SEF 22

SEP 22183

SF 23180

Eash. Bise San Luir Ints. 
2129

2430

2131 Solidago

2132 Quercus

2133 Quereus

2434 Salizi

2135 Pentsteunon

2436 Solidago

$243 \%$ Solidago

2435

2339 Quercur hypolenca

2410 Erigeron

2111 Quercus
SEF $24 \quad 1893$

SEP $24 \quad 189$

SEP $24 \quad 1893$

SEP 241893

West side San Luis Mts
$6150 \mathrm{fs}$

SEP $20 \quad 189$

SEP $20 \quad 1893$

SEP $26 \cdot 189$

SEP 26

SEP 26

SEP $25 \quad 1893$

Sก. $26 \quad 1899$

SEP 261893

SEP $26 \quad 1093$ sh side Sau Lais Ht.

.

西 


\section{$21 \%$}

2133 Gerainm

2111 Inaphalim

2135 Bidus

2116

2418

2115 Euthanina

2119 jauschuria

2450 Phus

2151 Monarda

2452

2453

2151 Dinabilio
SEP $26 \quad 189$

SEF $26 \quad 189$

Set $26 \quad 1995$

sep

ste $26 \quad 1899$

SEP $26 \quad 189$ !

SEF 26 189:

$\operatorname{SeP} 25 \quad 189$

$8526 \quad 1898$

SEP $26 \quad 1898$

SEP $20 \quad 18$

Seक $26 \quad 189$

$\operatorname{sep} 26 \quad 1899$

SeP $25 \quad 18 !$ lest side San Lun Mts. 
215.) Celenatir liquaticifolin Muts.

2156 Pentitemon

24.5

205 Garduns

2159 bastillya

2100 Jilia

2461

2182 Fallugia

2463 Froclichia

210 Eriocarpun

$2 \%(0.5)$ Lithospermun

2106 A A ter

2467

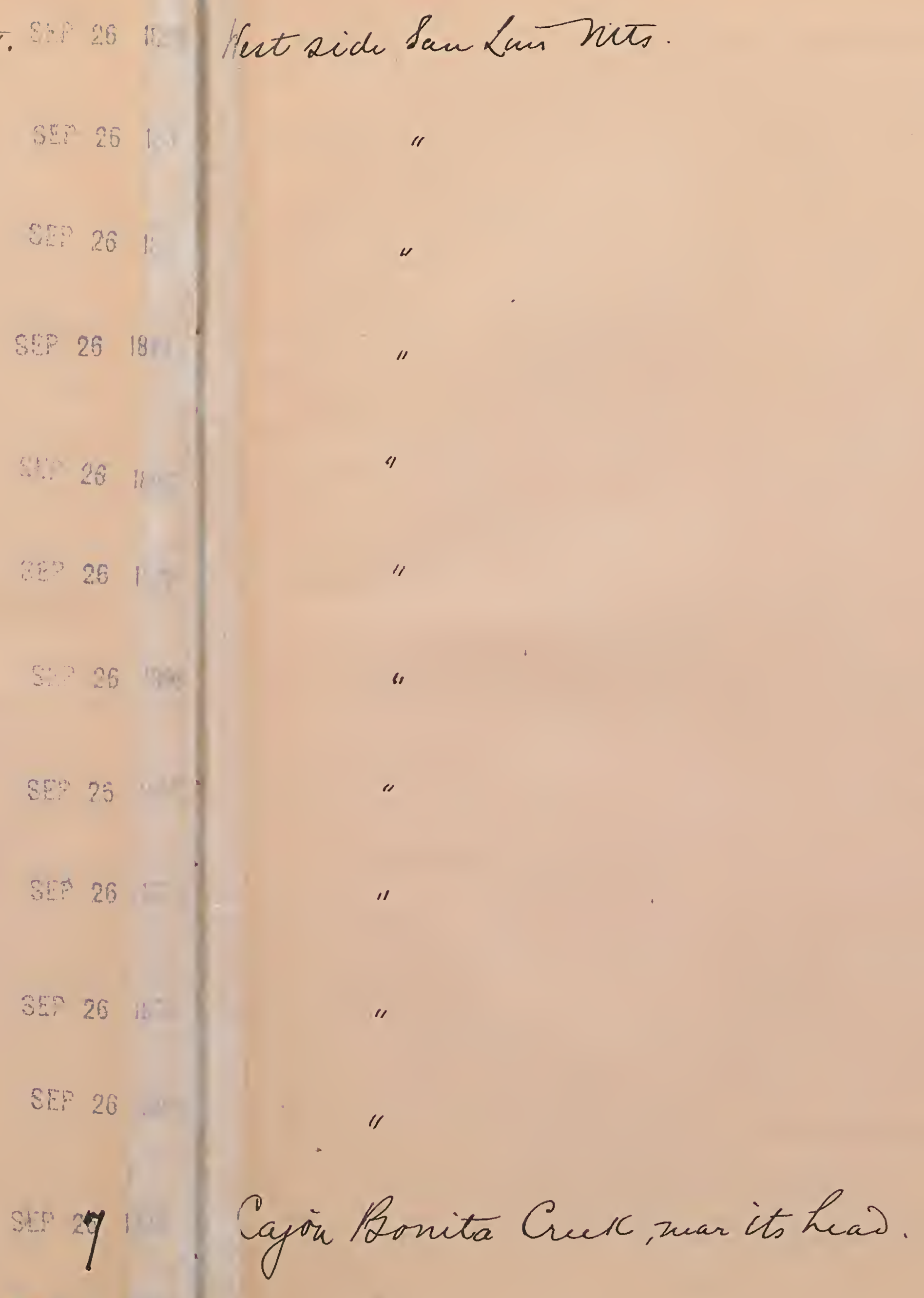


2465

$2 \% 69$

24:0

$21 \% 1$

2172 Lolanum vostratum

2483

2181 Artenisia

2185 Grotalaria

2486

217:

2135

2479 Phoradudron

2880 Quercus
SEP 27 Cajou Bonita lonek mar it head

SEP 27

SEP 27

SEP $27 \quad 189$

$\operatorname{seg} 27$ thas

SeP 271895

SEP 27 189:

$\operatorname{Lep} .29 .1893$

West side Lau Luis Nuts.

Lep. 30,18

OCT +18

Hachuca hat. Ariz.

(F.X. Holgner) 8500fr. or 1199

DOT 1 189S

$\cot 1 \tan$ 
$2491 \mathrm{Aar}$

248? Quercus

2483 Qurcus hypolunca

2454

2455 Phoradendror

2156 Verbena

245\% Berlaudiera

21SS Eniogoumur

2459 Pectis

2390 Sporobolus

2191 Saura

2492

2393
CoT 1. 1893

COT 1 igs

oct 1 18:

oct 1908

OCT 1 TB:H

OCT 2 HBS

OCT $2 \quad 1803$

OCT 2 189:

OCT 2 II

OCT 2 18:

DCT 2

$\cot 2$ ynas

UCt 2 IE
Vest side JauLuir Ho - alt 5675 fo

San Luis Caũon, Sau Luis Kuts_alr. $\$ 500^{\circ}$.

aet 5775 fs

alt. $5500 \mathrm{fs}$

als. 5575 fis.

thinas. Vally, New Mexico. 


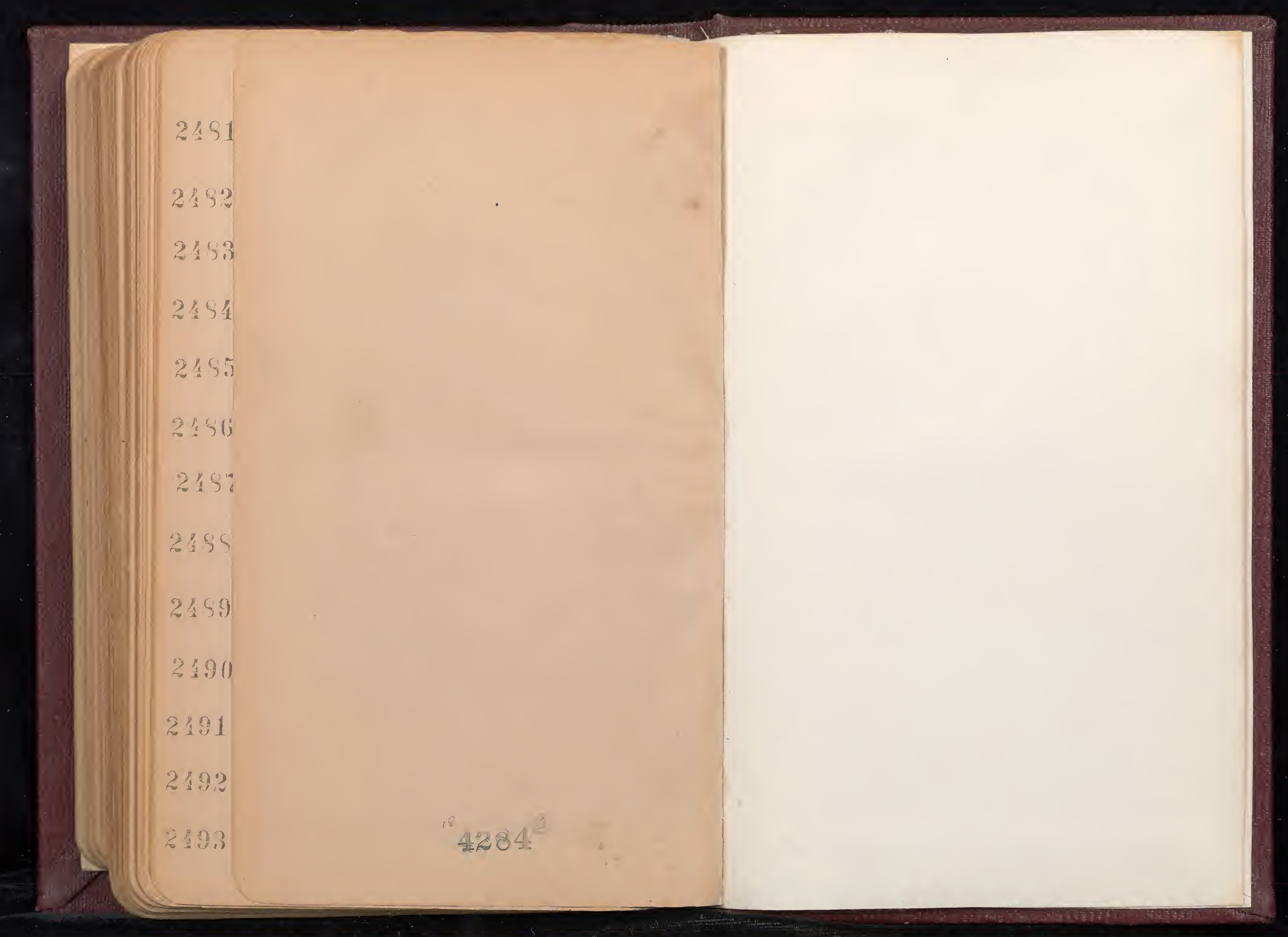




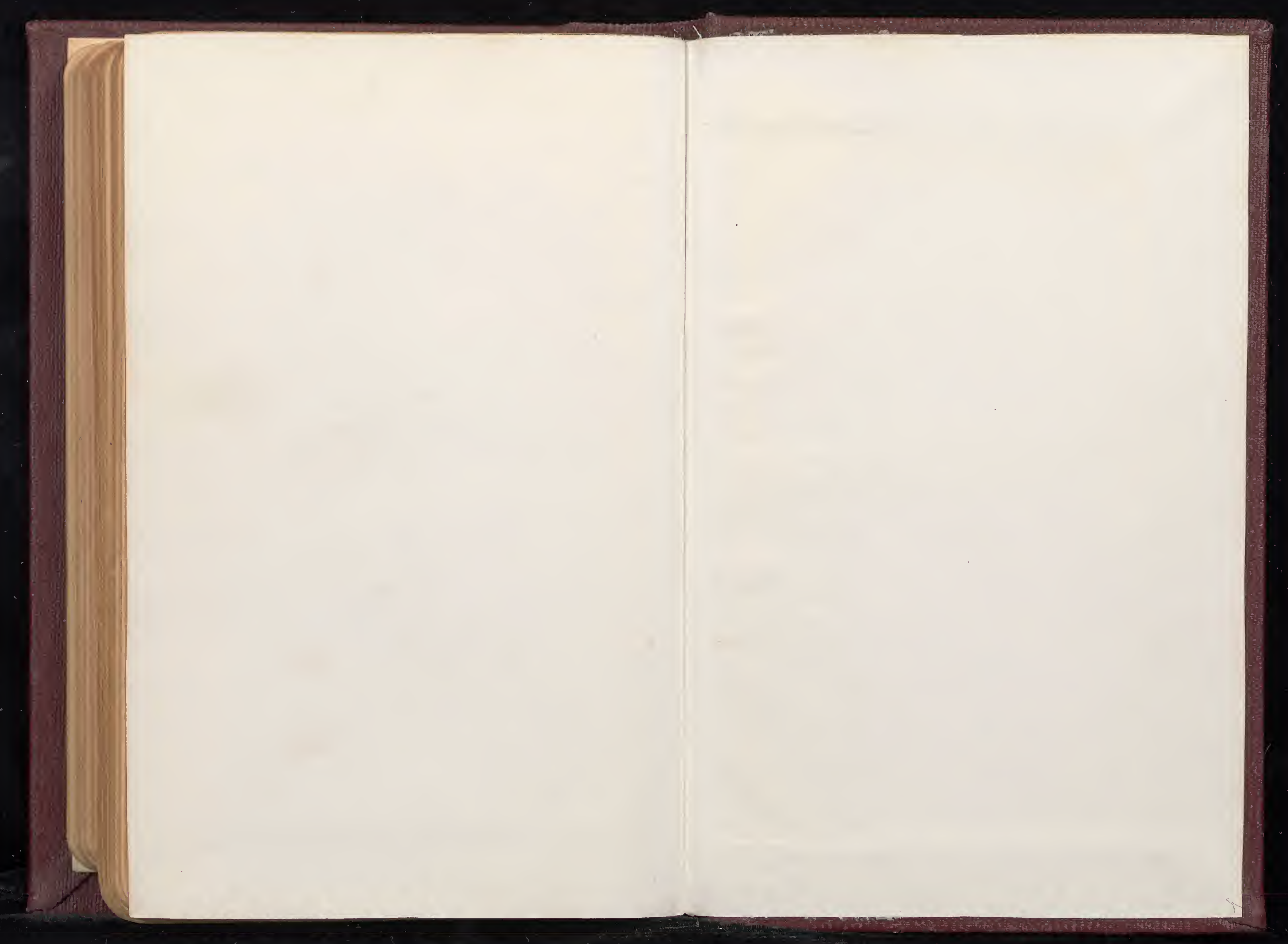




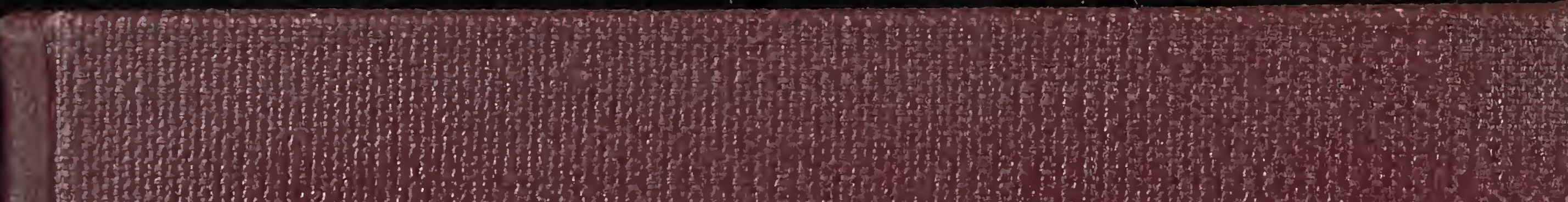
(5)

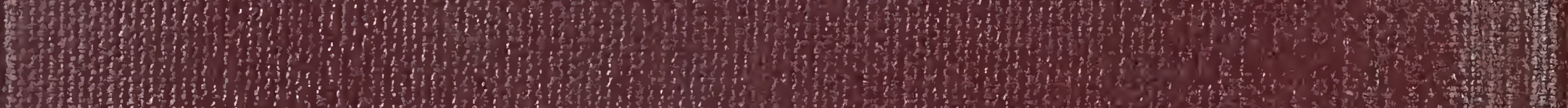
(1)

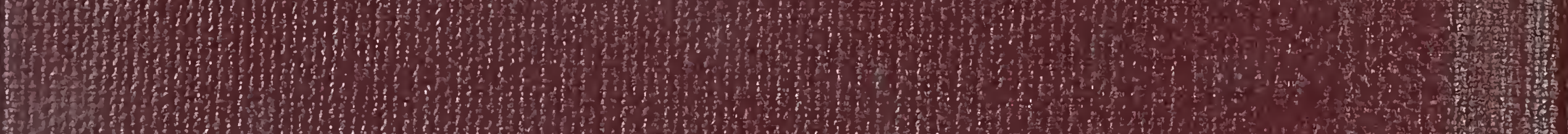

W.

Whin 